Pacific

Journal of

Mathematics

A FLOER HOMOLOGY FOR EXACT CONTACT EMBEDDINGS

Kai Cieliebak ANd URS AdRian Frauenfelder

Volume $239 \quad$ No. 2

February 2009 


\title{
A FLOER HOMOLOGY FOR EXACT CONTACT EMBEDDINGS
}

\author{
Kai Cieliebak And Urs Adrian Frauenfelder
}

\begin{abstract}
In this paper we construct the Floer homology for an action functional which was introduced by Rabinowitz and prove a vanishing theorem. As an application, we show that there are no displaceable exact contact embeddings of the unit cotangent bundle of a sphere of dimension greater than three into a convex exact symplectic manifold with vanishing first Chern class. This generalizes Gromov's result that there are no exact Lagrangian embeddings of a sphere into $\mathbb{C}^{n}$.
\end{abstract}

\section{Introduction}

Exact convex symplectic manifolds and hypersurfaces. An exact convex symplectic manifold $(V, \lambda)$ is a connected manifold $V$ of dimension $2 n$ without boundary with a 1 -form $\lambda$ such that the following conditions are satisfied.

(i) The 2-form $\omega=d \lambda$ is symplectic.

(ii) The symplectic manifold $(V, \omega)$ is convex at infinity, that is, there exists an exhaustion $V=\bigcup_{k} V_{k}$ of $V$ by compact sets $V_{k} \subset V_{k+1}$ with smooth boundary such that $\left.\lambda\right|_{\partial V_{k}}$ is a contact form.

(See [Eliashberg and Gromov 1991].) Define a vector field $Y_{\lambda}$ on $V$ by $i_{Y_{\lambda}} \omega=\lambda$. Then the last condition is equivalent to saying that $Y_{\lambda}$ points out of $V_{k}$ along $\partial V_{k}$.

We say that an exact convex symplectic manifold $(V, \lambda)$ is complete if the vector field $Y_{\lambda}$ is complete. We say that $(V, \lambda)$ has bounded topology if $Y_{\lambda} \neq 0$ outside a compact set. Note that $(V, \lambda)$ is complete and of bounded topology if and only if there exists an embedding $\phi: M \times \mathbb{R}_{+} \rightarrow V$ such that $\phi^{*} \lambda=e^{r} \alpha_{M}$ with contact form $\alpha_{M}=\left.\phi^{*} \lambda\right|_{M \times\{0\}}$, and such that $V \backslash \phi\left(M \times \mathbb{R}_{+}\right)$is compact. (To see this, simply apply the flow of $Y_{\lambda}$ to $M:=\partial V_{k}$ for large $k$ ).

We say that a subset $A \subset V$ is displaceable if it can be displaced from itself via a Hamiltonian isotopy, that is,there exists a smooth time-dependent Hamiltonian $H \in C^{\infty}([0,1] \times V)$ with compact support such that the time one flow $\phi_{H}$ of the time-dependent Hamiltonian vector field $X_{H_{t}}$ defined by $d H_{t}=-\iota_{X_{H_{t}}} \omega$ for $H_{t}=H(t, \cdot) \in C^{\infty}(V)$ and $t \in[0,1]$ satisfies $\phi_{H}(A) \cap A=\varnothing$.

MSC2000: 53D10, 53D40.

Keywords: contact manifolds, Floer homology, Rabinowitz action functional. 
The main examples of exact convex symplectic manifolds we have in mind are Stein manifolds. We briefly recall the definition. A Stein manifold is a triple $(V, J, f)$ where $V$ is a connected manifold, $J$ is an integrable complex structure on $V$ and $f \in C^{\infty}(V)$ is an exhausting plurisubharmonic function, that is, $f$ is proper and bounded from below, and the exact 2-form $\omega=-d d^{c} f$ is symplectic. Here the 1-form $\lambda=-d^{c} f$ is defined by the condition $d^{c} f(\xi)=d f(J \xi)$ for every vector field $\xi$. We refer to [Eliashberg 1990] for basic notions on Stein manifolds and Eliashberg's topological characterization of them. It is well known (loc. cit.) that if the plurisubharmonic function $f$ is Morse, then all critical points of $f$ have Morse index less than or equal than half the dimension of $V$. The Stein manifold $(V, J, f)$ is called subcritical if this inequality is strict. In a subcritical Stein manifold, every compact subset $A$ is displaceable [Biran and Cieliebak 2002, Lemma 3.2].

Remark. Examples of exact convex symplectic manifolds which are not Stein can be obtained using the following construction. Let $M$ be a $(2 n-1)$-dimensional closed manifold, $n \geq 2$, which admits a pair of contact forms $\left(\alpha_{0}, \alpha_{1}\right)$ satisfying

$$
\begin{gathered}
\alpha_{1} \wedge\left(d \alpha_{1}\right)^{n-1}=-\alpha_{0} \wedge\left(d \alpha_{0}\right)^{n-1}>0, \\
\alpha_{i} \wedge\left(d \alpha_{i}\right)^{k} \wedge\left(d \alpha_{j}\right)^{n-k-1}=0, \quad 0 \leq k \leq n-2
\end{gathered}
$$

where $(i, j)$ is a permutation of $(0,1)$. Then a suitable interpolation of $\alpha_{0}$ and $\alpha_{1}$ endows the manifold $V=M \times[0,1]$ with the structure of an exact convex symplectic manifold, where the restriction of the 1-form to $M \times\{0\}$ is given by $\alpha_{0}$ and the restriction to $M \times\{1\}$ is given by $\alpha_{1}$. Since $H_{2 n-1}(V)=\mathbb{Z}$, the manifold $V$ does not admit a Stein structure. Moreover, what makes these examples particularly interesting is the fact that they have two boundary components, whereas the boundary of a connected Stein manifold is always connected. The first construction in dimension four of an exact convex symplectic manifold of the type above was carried out by D. McDuff [1991]. H. Geiges generalized her method [1994], where he also obtained higher dimensional examples.

If $(V, \lambda)$ is an exact convex symplectic manifold then so is its stabilization $(V \times$ $\left.\mathbb{C}, \lambda \oplus \lambda_{\mathbb{C}}\right)$ for the 1 -form $\lambda_{\mathbb{C}}=\frac{1}{2}(x d y-y d x)$ on $\mathbb{C}$. Moreover, in $\left(V \times \mathbb{C}, \lambda \oplus \lambda_{\mathbb{C}}\right)$ every compact subset $A$ is displaceable. It is shown in [Cieliebak 2002b] that each subcritical Stein manifold is Stein deformation equivalent to a split Stein manifold, that is, a Stein manifold of the form $\left(V \times \mathbb{C}, J \times i, f+|z|^{2}\right)$ for a Stein manifold $(V, J, f)$.

Remark. If $(V, \lambda)$ is an exact convex symplectic manifold, then so is $(V, \lambda+d h)$ for any smooth function $h: V \rightarrow \mathbb{R}$ with compact support. We call the 1 -forms $\lambda$ and $\lambda+d h$ equivalent. For all our considerations only the equivalence class of $\lambda$ will be relevant. 
An exact convex hypersurface in an exact convex symplectic manifold $(V, \lambda)$ is a compact connected hypersurface (without boundary) $\Sigma \subset V$ such that:

(i) There exists a contact 1 -form $\alpha$ on $\Sigma$ such that $\alpha-\left.\lambda\right|_{\Sigma}$ is exact.

(ii) $\Sigma$ is bounding, that is, $V \backslash \Sigma$ consists of two connected components, one relatively compact and the other one not.

Remarks. (1) It follows that the volume form $\alpha \wedge(d \alpha)^{n-1}$ defines the orientation of $\Sigma$ as boundary of the bounded component of $V \backslash \Sigma$.

(2) If $\Sigma$ is an exact convex hypersurface in $(V, \lambda)$ with contact form $\alpha$, then there exists an equivalent 1-form $\mu=\lambda+d h$ on $V$ such that $\alpha=\left.\mu\right|_{\Sigma}$. To see this, extend $\alpha$ to a 1 -form $\beta$ on $V$. As $\left.(\beta-\lambda)\right|_{\Sigma}$ is exact, there exists a function $h$ on a neighbourhood $U$ of $\Sigma$ such that $\beta-\lambda=d h$ on $U$. Now simply extend $h$ to a function with compact support on $V$ and set $\mu:=\lambda+d h$.

(3) If $H^{1}(\Sigma ; \mathbb{R})=0$ condition (i) is equivalent to $d \alpha=\left.\omega\right|_{\Sigma}$.

(4) Condition (ii) is automatically satisfied if $H_{2 n-1}(V ; \mathbb{Z})=0$, for example if $V$ is a stabilization or a Stein manifold of dimension $>2$. Indeed, $V \backslash \Sigma$ has at most two connected components since $V$ is connected. If $V \backslash \Sigma$ is connected pick a small embedded arc in $V$ intersecting $\Sigma$ once transversally and connect its endpoints in $V \backslash \Sigma$; the resulting loop has homological intersection number \pm 1 with $\Sigma$, which implies $0 \neq[\Sigma] \in H_{2 n-1}(V ; \mathbb{Z})$.

Rabinowitz Floer homology. In the following we assume that $(V, \lambda)$ is a complete exact convex symplectic manifold of bounded topology, and $\Sigma \subset V$ is an exact convex hypersurface with contact form $\alpha$. We will define an invariant $R F H(\Sigma, V)$ as the Floer homology of an action functional which was studied previously by Rabinowitz [1978].

A defining Hamiltonian for $\Sigma$ is a function $H \in C^{\infty}(V)$ which is constant outside of a compact set of $V$, whose zero level set $H^{-1}(0)$ equals $\Sigma$, and whose Hamiltonian vector field $X_{H}$ defined by $d H=-\iota_{X_{H}} \omega$ agrees with the Reeb vector field $R$ of $\alpha$ on $\Sigma$. Defining Hamiltonians exist since $\Sigma$ is bounding, and they form a convex space.

Fix a defining Hamiltonian $H$ and denote by $\mathscr{L}=C^{\infty}(\mathbb{R} / \mathbb{Z}, V)$ the free loop space of $V$. The Rabinowitz action functional

$$
\mathscr{A}^{H}: \mathscr{L} \times \mathbb{R} \rightarrow \mathbb{R}
$$

is defined as

$$
\mathscr{A}^{H}(v, \eta):=\int_{0}^{1} v^{*} \lambda-\eta \int_{0}^{1} H(v(t)) d t, \quad(v, \eta) \in \mathscr{L} \times \mathbb{R} .
$$


One may think of $\mathscr{A}^{H}$ as the Lagrange multiplier functional of the unperturbed action functional of classical mechanics also studied in Floer theory to a mean value constraint of the loop. The critical points of $\mathscr{A}^{H}$ satisfy

$$
\left\{\begin{aligned}
\partial_{t} v(t) & =\eta X_{H}(v(t)), \quad t \in \mathbb{R} / \mathbb{Z} \\
H(v(t)) & =0
\end{aligned}\right.
$$

Here we used the fact that $H$ is invariant under its Hamiltonian flow. Since the restriction of the Hamiltonian vector field $X_{H}$ to $\Sigma$ is the Reeb vector field, the equations (2) are equivalent to

$$
\left\{\begin{aligned}
\partial_{t} v(t) & =\eta R(v(t)), & & t \in \mathbb{R} / \mathbb{Z}, \\
v(t) & \in \Sigma, & & t \in \mathbb{R} / \mathbb{Z},
\end{aligned}\right.
$$

that is, $v$ is a periodic orbit of the Reeb vector field on $\Sigma$ with period $\eta .^{1}$

Theorem 1.1. Under the above hypotheses, the Floer homology $H F\left(A^{H}\right)$ is welldefined. Moreover, if $H_{s}$ for $0 \leq s \leq 1$ is a smooth family of defining functions for exact convex hypersurfaces $\Sigma_{s}$, then $H F\left(\mathscr{A}^{H_{0}}\right)$ and $H F\left(\mathscr{A}^{H_{1}}\right)$ are canonically isomorphic.

Hence the Floer homology $H F\left(\mathscr{A}^{H}\right)$ is independent of the choice of the defining Hamiltonian $H$ for an exact convex hypersurface $\Sigma$, and the resulting Rabinowitz Floer homology

$$
R F H(\Sigma, V):=H F\left(\mathscr{A}^{H}\right)
$$

does not change under homotopies of exact convex hypersurfaces.

The next result is a vanishing theorem for the Rabinowitz Floer homology $\operatorname{RFH}(\Sigma, V)$.

Theorem 1.2. If $\Sigma$ is displaceable, then $\operatorname{RFH}(\Sigma, V)=0$.

Remark. The action functional $\mathscr{A}^{H}$ is also defined if $H^{-1}(0)$ is not exact convex. However, in this case the Rabinowitz Floer homology $R F H\left(\mathscr{A}^{H}\right)$ cannot in general be defined because the moduli spaces of flow lines will in general not be compact up to breaking anymore. The problem is that the Lagrange multiplier $\eta$ may go to infinity. This phenomenon actually does happen as the counterexamples to the Hamiltonian Seifert conjecture show; see [Ginzburg and Gürel 2003] and the literature cited therein.

Denote by $c_{1}$ the first Chern class of the tangent bundle of $V$ (with respect to an $\omega$-compatible almost complex structure and independent of this choice; see [McDuff and Salamon 1998, Theorem 2.69]). Evaluation of $c_{1}$ on spheres gives rise to a

\footnotetext{
${ }^{1}$ The period $\eta$ may be negative or zero. We refer in this paper to Reeb orbits moved backwards as Reeb orbits with negative period and to constant orbits as Reeb orbits of period zero.
} 
homomorphism $I_{c_{1}}: \pi_{2}(V) \rightarrow \mathbb{Z}$. If $I_{c_{1}}$ vanishes then the Rabinowitz Floer homology $R F H_{*}(\Sigma, V)$ can be $\mathbb{Z}$-graded with half integer degrees, that is, $* \in 1 / 2+\mathbb{Z}$.

The third result is a computation of Rabinowitz Floer homology (with $\mathbb{Z}_{2^{-}}$ coefficients) for the unit cotangent bundle of a sphere.

Theorem 1.3. Let $(V, \lambda)$ be a complete exact convex symplectic manifold of bounded topology satisfying $I_{c_{1}}=0$. Suppose that $\Sigma \subset V$ is an exact convex hypersurface with contact form $\alpha$ such that $(\Sigma, \operatorname{ker} \alpha)$ is contactomorphic to the unit cotangent bundle $S^{*} S^{n}$ of the sphere of dimension $n \geq 4$ with its standard contact structure. Then

$$
\operatorname{RFH}_{k}(\Sigma, V)=\left\{\begin{array}{cl}
\mathbb{Z}_{2}, & k \in\left\{-n+\frac{1}{2},-\frac{1}{2}, \frac{1}{2}, n-\frac{1}{2}\right\}+\mathbb{Z} \cdot(2 n-2), \\
0, & \text { else. }
\end{array}\right.
$$

Applications and discussion. The following well-known technical lemma will allow us to remove completeness and bounded topology from the hypotheses of our corollaries.

Lemma 1.4. Assume that $\Sigma$ is an exact convex hypersurface in the exact convex symplectic manifold $(V, \lambda)$. Then $V$ can be modified outside of $\Sigma$ to an exact convex symplectic manifold $(\hat{V}, \hat{\lambda})$ which is complete and of bounded topology. If $I_{c_{1}}=0$ for $V$ the same holds for $\hat{V}$. If $\Sigma$ is displaceable in $V$, then we can arrange that it is displaceable in $\widehat{V}$ as well.

Proof. Let $V_{1} \subset V_{2} \ldots$ be the compact exhaustion in the definition of an exact convex symplectic manifold. Since $\Sigma$ is compact, it is contained in $V_{k}$ for some $k$. The flow of $Y_{\lambda}$ for times $r \in(-1,0]$ defines an embedding $\phi: \partial V_{k} \times(-1,0] \rightarrow V_{k}$ such that $\phi^{*} \lambda=e^{r} \lambda_{0}$, where $\lambda_{0}=\left.\lambda\right|_{\partial V_{k}}$. Now define

$$
(\hat{V}, \hat{\lambda}):=\left(V_{k}, \lambda\right) \cup_{\phi}\left(\partial V_{k} \times(-1, \infty), e^{r} \lambda_{0}\right) .
$$

This is clearly complete and of bounded topology. The statement about $I_{c_{1}}$ is obvious. If $\Sigma$ is displaceable by a Hamiltonian isotopy generated by a compactly supported Hamiltonian $H:[0,1] \times V \rightarrow \mathbb{R}$ we choose $k$ so large that $\operatorname{supp} H \subset$ $[0,1] \times V_{k}$ and apply the same construction.

As a first consequence of Theorem 1.2, we recover some known cases of the Weinstein conjecture; see [Viterbo 1999] and [Frauenfelder et al. 2005].

Corollary 1.5. Every displaceable exact convex hypersurface $\Sigma$ in an exact convex symplectic manifold $(V, \lambda)$ carries a closed characteristic. In particular, this applies to all exact convex hypersurfaces in a subcritical Stein manifold, or more generally in a stabilization $V \times \mathbb{C}$.

Proof. In view of Lemma 1.4, we may assume without loss of generality that $(V, \lambda)$ is complete and of bounded topology. Then by Theorem 1.2 the Floer homology 
$H F\left(\mathscr{A}^{H}\right)$ vanishes, where $H$ is a defining function for $\Sigma$. On the other hand, in the absence of closed characteristics the constant loops in $\Sigma$ give rise to $H F\left(\mathscr{A}^{H}\right) \cong$ $H(\Sigma) \neq 0$, contradicting the vanishing of $H F\left(\mathscr{A}^{H}\right)$.

For further applications, the following notation will be convenient. An exact contact embedding of a closed contact manifold $(\Sigma, \xi)^{2}$ into an exact convex symplectic manifold $(V, \lambda)$ is an embedding $\iota: \Sigma \rightarrow V$ such that:

(i) There exists a 1 -form $\alpha$ on $\Sigma$ such that $\operatorname{ker} \alpha=\xi$ and $\alpha-\iota^{*} \lambda$ is exact.

(ii) The image $\iota(\Sigma) \subset V$ is bounding.

In other words, $\iota(\Sigma) \subset V$ is an exact convex hypersurface with contact form $\iota_{*} \alpha$ which is contactomorphic (via $\left.\iota^{-1}\right)$ to $(\Sigma, \xi)$.

Now Theorems 1.2 and 1.3 together with Lemma 1.4 immediately imply:

Corollary 1.6. Assume that $n \geq 4$ and there exists an exact contact embedding $\iota$ of $S^{*} S^{n}$ into an exact convex symplectic manifold satisfying $I_{c_{1}}=0$. Then $\iota\left(S^{*} S^{n}\right)$ is not displaceable.

Since in a stabilization $V \times \mathbb{C}$ all compact subsets are displaceable, we obtain in particular:

Corollary 1.7. For $n \geq 4$ there does not exist an exact contact embedding of $S^{*} S^{n}$ into a subcritical Stein manifold, or more generally, into the stabilization $(V \times$ $\mathbb{C}, \lambda \oplus \lambda \mathbb{C})$ of an exact convex symplectic manifold $(V, \lambda)$ satisfying $I_{c_{1}}=0$.

Remark. If $n$ is even then there are no smooth embeddings of $S^{*} S^{n}$ into a subcritical Stein manifold for topological reasons; see Appendix D. However, at least for $n=3$ and $n=7$ there are no topological obstructions; see the discussion below.

If $(V, J, f)$ is a Stein manifold with $f$ a Morse function, P. Biran [2006] defines the critical coskeleton as the union of the unstable manifolds (with respect to $\nabla f$ ) of the critical points of index $\operatorname{dim} V / 2$. It is proved in [Biran 2006, Lemma 2.4.A] that every compact subset $A \subset V$ which does not intersect the critical coskeleton is displaceable. For example, in a cotangent bundle the critical coskeleton (after a small perturbation) is one given fibre. Thus Corollary 1.6 implies:

Corollary 1.8. Assume that $n \geq 4$ and there exists an exact contact embedding $\mathrm{c}$ of $S^{*} S^{n}$ into a Stein manifold $(V, J, f)$ satisfying $I_{c_{1}}=0$. Then $\iota(\Sigma)$ must intersect the critical coskeleton. In particular, the image of an exact contact embedding of $S^{*} S^{n}$ into a cotangent bundle $T^{*} Q$ must intersect every fibre.

Remark. Let $\iota: L \rightarrow V$ be an exact Lagrangian embedding of $L$ into $V$, that is, such that $\iota^{*} \lambda$ is exact. Since by Weinstein's Lagrangian neighbourhood theorem [McDuff and Salamon 1998, Theorem 3.33] a tubular neighbourhood of $\iota(L)$ can

${ }^{2}$ All contact structures are assumed to be cooriented 
be symplectically identified with a tubular neighbourhood of the zero section in the cotangent bundle of $L$, we obtain an exact contact embedding of $S^{*} L$ into $V$. Thus the last three corollaries generalize corresponding results about exact Lagrangian embeddings. For example, Corollary 1.7 generalizes (for spheres) the well-known result [Gromov 1985; Audin et al. 1994; Viterbo 1999; Biran and Cieliebak 2002] that there exist no exact Lagrangian embeddings into subcritical Stein manifolds. Corollary 1.8 implies (see [Biran 2006]) that an embedded Lagrangian sphere of dimension $\geq 4$ in a cotangent bundle $T^{*} Q$ must intersect every fibre.

Remark. Let us discuss Corollary 1.7 in the cases $n \leq 3$ that are not accessible by our method of proof. We always equip $\mathbb{C}^{n}$ with the canonical 1 -form $\lambda=$ $\frac{1}{2} \sum_{i=1}^{n}\left(x_{i} d y_{i}-y_{i} d x_{i}\right)$.

$n=1$ : Any embedding of two disjoint circles into $\mathbb{C}$ is an exact contact embedding of $S^{*} S^{1}$, so Corollary 1.7 fails in this case.

$n=2$ : In this case Corollary 1.7 is true for purely topological reasons; we present various proofs in Appendix D.

$n=3$ : In this case Corollary 1.7 is true for subcritical Stein manifolds and can be proved using symplectic homology; see the last remark in this section.

Example. In this example we illustrate that the preceding results about exact contact embeddings are sensitive to the contact structure. Let $n=3$ or $n=7$. Then $S^{*} S^{n} \cong S^{n} \times S^{n-1}$ embeds into $\mathbb{R}^{n+1} \times S^{n-1}$. On the other hand $\mathbb{R}^{n+1} \times S^{n-1}$ is diffeomorphic to the subcritical Stein manifold $T^{*} S^{n-1} \times \mathbb{C}$, and identifying $S^{*} S^{n}$ with a level set in $T^{*} S^{n-1} \times \mathbb{C}$ defines a contact structure $\xi_{\text {subcrit }}$ on $S^{*} S^{n}$. Thus $\left(S^{*} S^{n}, \xi_{\text {subcrit }}\right)$ has an exact contact embedding into a subcritical Stein manifold (in fact into $\mathbb{C}^{n}$ ) for $n=3,7$, whereas $\left(S^{*} S^{7}, \xi_{\text {st }}\right)$ admits no such embedding by Corollary 1.7. In particular, we conclude:

Corollary 1.9. The two contact structures $\xi_{\text {subcrit }}$ and $\xi_{\text {st }}$ on $S^{*} S^{7} \cong S^{7} \times S^{6}$ described above are not diffeomorphic.

Remarks. (1) The contact structures $\xi_{\text {subcrit }}$ and $\xi_{\text {st }}$ on $S^{*} S^{7} \cong S^{7} \times S^{6}$ are also distinguished by their cylindrical contact homology. This follows from a result by M.-L. Yau [2004] for $\xi_{\text {subcrit }}$ and a direct computation for $\xi_{\text {st }}$; see Appendix E.

(2) Corollary 1.9 also holds in the case $n=3$, although our method does not apply there. This follows, for example, from the main result in [Cieliebak et al. 2008]. As pointed out by O. van Koert, the contact structures $\xi_{\text {subcrit }}$ and $\xi_{\text {st }}$ on $S^{*} S^{3} \cong S^{3} \times S^{2}$ are not distinguished by their cylindrical contact homology; see Appendix E.

(3) The contact structures $\xi$ and $\xi_{\text {st }}$ on $S^{3} \times S^{2}$ are homotopic as almost contact structures, that is, as symplectic hyperplane distributions. This follows simply 
from the fact (see for example [Geiges 2008, Proposition 9.1.1]) that on closed oriented 5-manifolds almost contact structures are classified up to homotopy by their first Chern classes and $c_{1}(\xi)=c_{1}\left(\xi_{\text {st }}\right)=0$. It would be interesting to know whether $\xi$ and $\xi_{\text {st }}$ on $S^{7} \times S^{6}$ are also homotopic as almost contact structures. Here the first obstruction to such a homotopy vanishes because $c_{3}(\xi)=c_{3}\left(\xi_{\mathrm{st}}\right)=0$, but there are further obstructions in dimensions 7 and 13 which remain to be analysed along the lines of [Morita 1975].

Remark (Obstructions from symplectic field theory). Symplectic field theory (see [Eliashberg et al. 2000]) also yields obstructions to exact contact embeddings. For example, by neck stretching along the image of an exact contact embedding, the following result can be deduced using the techniques in [Cieliebak and Mohnke 2004]:

Let $\left(\Sigma^{2 n-1}, \xi\right)$ be a closed contact manifold with $H_{1}(\Sigma ; \mathbb{Z})=0$ which admits an exact contact embedding into $\mathbb{C}^{n}$. Then for every nondegenerate contact form defining $\xi$ there exist closed Reeb orbits of Conley-Zehnder indices $n+1+2 k$ for all integers $k \geq 0$.

Here Conley-Zehnder indices are defined with respect to trivializations extending over spanning surfaces. This result applies in particular to the unit cotangent bundle $\Sigma=S^{*} Q$ of a closed Riemannian manifold $Q$ with $H_{1}(Q ; \mathbb{Z})=0$. For example, if $Q$ carries a metric of nonpositive curvature then all indices are $\leq n-1$ and hence $S^{*} Q$ admits no exact contact embedding into $\mathbb{C}^{n}$. On the other hand, any nondegenerate metric on the sphere $S^{n}$ has closed geodesics of all indices $n+1+2 k, k \geq 0$, so this result does not exclude exact contact embeddings $S^{*} S^{n} \hookrightarrow \mathbb{C}^{n}$.

Remark (Obstructions from symplectic homology). Corollary 1.7 for subcritical Stein manifolds can be proved for all $n \geq 3$ by combining the following five results on symplectic homology. See [Cieliebak and Oancea 2008] for details.

(1) The symplectic homology $S H(V)$ of a subcritical Stein manifold $V$ vanishes [Cieliebak 2002a].

(2) If $\Sigma \subset V$ is an exact convex hypersurface in an exact convex symplectic manifold bounding the compact domain $W \subset V$, then $S H(V)=0$ implies $S H(W)=0$. This follows from an argument by M. McLean [2007], based on Viterbo's transfer map [Viterbo 1999] and the ring structure on symplectic homology.

(3) If $S H(W)=0$, then the positive action part $S^{+}(W)$ of symplectic homology is only nonzero in finitely many degrees. This follows from the long exact sequence induced by the action filtration. 
(4) $\mathrm{SH}^{+}(W)$ equals the nonequivariant linearized contact homology $\mathrm{NCH}(W)$. This is implicit in [Bourgeois and Oancea 2007]; see also [Cieliebak and Oancea 2008].

(5) If $\partial W=S^{*} S^{n}$ and $n \geq 3$, then $N C H(W)$ is independent of the exact filling $W$ and equals the homology of the free loop space of $S^{n}$ (modulo the constant loops), which is nonzero in infinitely many degrees.

\section{Exact contact embeddings}

Let $\Sigma$ be a connected closed ( $2 n-1)$-dimensional manifold. A contact structure $\xi$ is a field of hyperplanes $\xi \subset T \Sigma$ such that there exists a 1-form $\alpha$ satisfying

$$
\xi=\operatorname{ker} \alpha, \quad \alpha \wedge d \alpha^{n-1}>0 .
$$

The 1-form $\alpha$ is called a contact form. It is determined by $\xi$ up to multiplication with a function $f>0$. Given a contact form $\alpha$ the Reeb vector field $R$ on $\Sigma$ is defined by the conditions

$$
\iota_{R} d \alpha=0, \quad \alpha(R)=1 .
$$

Unit cotangent bundles have a natural contact structure as the following example shows.

Example. For a manifold $N$ we denote by $S^{*} N$ the oriented projectivization of its cotangent bundle $T^{*} N$, that is, elements of $S^{*} N$ are equivalence classes [ $v^{*}$ ] of cotangent vectors $v^{*} \in T^{*} N$ under the equivalence relation $v^{*} \cong w^{*}$ if there exists $r>0$ such that $v^{*}=r w^{*}$. Denote by $\pi: S^{*} N \rightarrow N$ the canonical projection. A contact structure $\xi$ on $S^{*} N$ is given by

$$
\xi_{\left[v^{*}\right]}=\operatorname{ker}\left(v^{*} \circ d \pi\right) .
$$

If $g$ is a Riemannian metric on $N$ then $S^{*} N$ can be identified with the space of tangent vectors of $N$ of length one and the restriction of the Liouville 1-form defines a contact form. Observe that the Reeb vector field generates the geodesic flow on $N$.

Consider an exact contact embedding $\iota:(\Sigma, \xi) \rightarrow(V, \lambda)$ such that $\alpha=\iota^{*} \lambda$ defines a contact form $\xi$. One might ask which contact forms $\alpha$ can arise in this way. The following proposition shows that if one contact form defining the contact structure $\xi$ arises from an exact contact embedding, then every other contact form defining $\xi$ arises as well.

Proposition 2.1. Assume that $\iota:(\Sigma, \xi) \rightarrow(V, \lambda)$ is an exact contact embedding with $\xi=\operatorname{ker} \iota * \lambda$. Then for every contact form $\alpha$ defining the contact structure $\xi$ on $\Sigma$ there exists a constant $c>0$ and a bounding embedding $\iota_{\alpha}: \Sigma \rightarrow V$ such that $\iota_{\alpha}^{*} \lambda=c \alpha$. 
Proof of Proposition 2.1. The proof uses the fact that if there exists an exact contact embedding of a contact manifold into an exact convex symplectic manifold $(V, \lambda)$ then the negative symplectization can be embedded. To see that we need two facts. Recall that the vector field $Y_{\lambda}$ is defined by the condition $\lambda=\iota_{Y_{\lambda}} d \lambda$.

Fact 1. The flow $\phi_{\lambda}^{t}$ of $Y_{\lambda}$ exists for all negative times $t$.

Indeed, let $x \in V$. Then $x \in V_{k}$ for some $k$. As $Y_{\lambda}$ points out of $V_{k}$ along $\partial V_{k}$, $\phi_{\lambda}^{t}(x) \in V_{k}$ for all $t \leq 0$ and compactness of $V_{k}$ yields completeness for $t \leq 0$.

Fact 2. The vector field $Y_{\lambda}$ satisfies

$$
\iota_{Y_{\lambda}} \lambda=0, \quad L_{Y_{\lambda}} \lambda=\lambda,
$$

where $L_{Y_{\lambda}}$ is the Lie derivative along the vector field $Y_{\lambda}$. In particular, the flow $\phi_{\lambda}^{r}$ of $Y_{\lambda}$ satisfies $\left(\phi_{\lambda}^{r}\right)^{*} \lambda=e^{r} \lambda$.

Indeed, the first equation in (4) follows directly from the definition of $Y_{\lambda}$. To prove the second one we compute using Cartan's formula

$$
L_{Y_{\lambda}} \lambda=d \iota_{Y_{\lambda}} \lambda+\iota_{Y_{\lambda}} d \lambda=\lambda \text {. }
$$

Now set $\alpha_{0}=\iota^{*} \lambda$ and consider the symplectic manifold $\left(\Sigma \times \mathbb{R}_{-}, d\left(e^{r} \alpha_{0}\right)\right)$ where $r$ denotes the coordinate on the $\mathbb{R}$-factor. By Fact 1 , the flow $\phi_{\lambda}^{r}$ exists for all $r \leq 0$. By Fact 2, the embedding

$$
\hat{\imath}: \Sigma \times \mathbb{R}_{-} \rightarrow V, \quad(x, r) \mapsto \phi_{\lambda}^{r}(\iota(x))
$$

satisfies

$$
(\hat{\iota})^{*} \lambda=e^{r} \alpha_{0} .
$$

If $\alpha$ is another contact form on $\Sigma$ which defines the contact structure $\xi$ then there exists a smooth function $\rho_{\alpha} \in C^{\infty}(\Sigma)$ such that

$$
\alpha=e^{\rho_{\alpha}} \alpha_{0} .
$$

Set $m:=\max _{\Sigma} \rho_{\alpha}$ and $c:=e^{-m}$. Then

$$
\iota_{\alpha}: \Sigma \rightarrow V, \quad x \mapsto \hat{\imath}\left(x, \rho_{\alpha}(x)-m\right)
$$

gives the required contact embedding for $\alpha$.

Remark. If the vector field $Y_{\lambda}$ is complete, then the preceding proof yields a symplectic embedding of the whole symplectization $\left(\Sigma \times \mathbb{R}, d\left(e^{r} \alpha_{0}\right)\right)$ into $(V, \omega)$. 


\section{Rabinowitz Floer homology}

In this section we construct the Floer homology for the Rabinowitz action functional defined in the introduction and prove Theorem 1.1 and Theorem 1.2. We assume that the reader is familiar with the constructions in Floer theory which can be found in Floer's original papers [1988a; 1988b; 1988c; 1989b; 1989a] or in Salamon's lectures [1999]. The finite dimensional case of Morse theory is treated in [Schwarz 1993].

Throughout this section we maintain the following setup:

- $(V, \lambda)$ is a complete exact convex symplectic manifold of bounded topology.

- $\Sigma \subset V$ is an exact convex hypersurface with contact form $\alpha$ and defining Hamiltonian $H$ such that $\alpha=\left.\lambda\right|_{\Sigma}$ and $X_{H}=R$ along $\Sigma$.

Our sign conventions for Floer homology are as follows:

- The Hamiltonian vector field $X_{H}$ is defined by $d H=-i_{X_{H}} \omega$, where $\omega=d \lambda$.

- An almost complex structure $J$ on $V$ is $\omega$-compatible if $\omega(\cdot, J \cdot)$ defines a Riemannian metric. Thus the gradient with respect to this metric is related to the symplectic vector field by $X_{H}=J \nabla H$.

- Floer homology is defined using the positive gradient flow of the action functional $\mathscr{A}^{H}$.

3.1. Compactness. In this subsection we prove that the moduli space of gradient flow lines of the Rabinowitz action functional of uniformly bounded energy is compact up to breaking of gradient flow lines. There are three difficulties one has to solve.

- An $L^{\infty}$-bound on the loop $v \in \mathscr{L}$.

- An $L^{\infty}$-bound on the Lagrange multiplier $\eta \in \mathbb{R}$.

- An $L^{\infty}$-bound on the derivatives of the loop $v$.

Although the first and the third point are nontrivial they are standard problems in Floer theory one knows how to deal with. The $L^{\infty}$-bound for the loop follows from the convexity assumption on $V$ and the derivatives can be controlled since our symplectic manifold is exact and hence there is no bubbling of holomorphic spheres. The new feature is the bound on the Lagrange multiplier $\eta$. We will explain in detail how this can be achieved. It will be essential that our hypersurface is convex.

We first derive the gradient flow equation for the Rabinowitz action functional. To compute the gradient we have to explain the metric on the space $\mathscr{L} \times \mathbb{R}$. The metric we choose on $\mathscr{L} \times \mathbb{R}$ is the product metric of the standard metric on $\mathbb{R}$ and a metric on $\mathscr{L}$ coming from a family of $\omega$-compatible almost complex structures $J_{t}$ 
on $V$. In order to deal with the $L^{\infty}$-bound for the loop we have to choose the almost complex structures compatible with the convex structure. As we remarked in the introduction, completeness implies that there exists a contact manifold $\left(M, \alpha_{M}\right)^{3}$ such that a neighbourhood of infinity of the symplectic manifold $(V, \omega)$ can be symplectically identified with $\left(M \times \mathbb{R}_{+}, d\left(e^{r} \alpha_{M}\right)\right)$, where $r$ refers to the coordinate on $\mathbb{R}_{+}=\{r \in \mathbb{R}: r \geq 0\}$. Since $H$ is constant outside of a compact set, we can further choose $M \times \mathbb{R}_{+}$in such a way that $X_{H}$ vanishes on it. We require that $J_{t}$ for every $t \in[0,1]$ is cylindrical on $M \times \mathbb{R}_{+}$in the following sense:.

- For each $x \in M$ we have $J_{t}(x) \frac{\partial}{\partial r}=R_{M}$, where $R_{M}$ is the Reeb vector field on $\left(M, \alpha_{M}\right)$.

- $J_{t}$ leaves the kernel of $\alpha_{M}$ invariant for every $x \in M$.

- $J_{t}$ is invariant under the local half flow $(x, 0) \mapsto(x, r)$ for $(x, r) \in M \times \mathbb{R}_{+}$.

For such a family of $\omega$-compatible almost complex structures $J_{t}$ we define the metric $g_{J}$ on $\mathscr{L} \times \mathbb{R}$ for $(v, \eta) \in \mathscr{L} \times \mathbb{R}$ and $\left(\hat{v}_{1}, \hat{\eta}_{1}\right),\left(\hat{v}_{2}, \hat{\eta}_{2}\right) \in T_{(v, \eta)}(\mathscr{L} \times \mathbb{R})$ by

$$
g_{J}\left(\left(\hat{v}_{1}, \hat{\eta}_{1}\right),\left(\hat{v}_{2}, \hat{\eta}_{2}\right)\right)=\int_{0}^{1} \omega\left(\hat{v}_{1}, J_{t}(v) \hat{v}_{2}\right) d t+\hat{\eta}_{1} \cdot \hat{\eta}_{2}
$$

The gradient of $\mathscr{A}^{H}$ with respect to this metric is given by

$$
\nabla \mathscr{A}^{H}=\nabla_{J} \mathscr{A}^{H}=\left(\begin{array}{c}
-J_{t}(v)\left(\partial_{t} v-\eta X_{H}(v)\right) \\
-\int_{0}^{1} H(v) d t
\end{array}\right) .
$$

Thus gradient flow lines of $\nabla \mathscr{A}^{H}$ are solutions $(v, \eta) \in C^{\infty}\left(\mathbb{R} \times S^{1}, V\right) \times C^{\infty}(\mathbb{R}, \mathbb{R})$ of the following problem

$$
\left\{\begin{array}{c}
\partial_{s} v+J_{t}(v)\left(\partial_{t} v-\eta X_{H}(v)\right)=0 \\
\partial_{s} \eta+\int_{0}^{1} H(v) d t=0 .
\end{array}\right.
$$

It is useful to also consider time-dependent Hamiltonians of the form

$$
H^{\chi}(t, x)=\chi(t) \bar{H}(x)
$$

for a defining Hamiltonian $H$ and a function $\chi \in C^{\infty}\left(S^{1}, \mathbb{R}\right)$ which satisfies

$$
\int_{0}^{1} \chi(t) d t=1
$$

We define the Rabinowitz action functional $\mathscr{A}^{H^{\chi}}$ as above with $H$ replaced by $H^{\chi}$. As the Hamiltonian vector field of $H^{\chi}$ is given by $X_{H^{\chi}}=\chi X_{H}$, critical

\footnotetext{
${ }^{3}$ Be careful! Do not confuse the contact manifolds $\left(M, \alpha_{M}\right)$ and $(\Sigma, \alpha)$.
} 
points of $\mathscr{A}^{H^{\chi}}$ satisfy the equations

$$
\left\{\begin{array}{c}
\partial_{t} v=\eta \chi(t) X_{H}(v) \\
\int_{0}^{1} \chi(t) H(v(t)) d t=0 .
\end{array}\right.
$$

The first equation shows that $v$ is a reparametrized periodic orbit of $X_{H}$. In particular, $H$ is constant along $v$ and the second equation yields $H(v(t)) \equiv 0$, so $v$ correspond is a reparametrized Reeb orbit on $\Sigma$. Thus critical points of $\mathscr{A}^{H^{x}}$ correspond to solutions of the problem

$$
\left\{\begin{array}{cc}
\partial_{t} v(t)=\eta \chi(t) R(v(t)), & t \in \mathbb{R} / \mathbb{Z}, \\
v(t) \in \Sigma, & t \in \mathbb{R} / \mathbb{Z} .
\end{array}\right.
$$

In particular, there is a natural one-to-one correspondence between critical points of the Rabinowitz action functionals $\mathscr{A}^{H^{\chi}}$ for fixed different choices of $\chi$.

The gradient flow equation for $\mathscr{A}^{H^{\chi}}$ becomes

$$
\left\{\begin{array}{c}
\partial_{s} v+J_{t}(v)\left(\partial_{t} v-\chi \eta X_{H}(v)\right)=0 \\
\partial_{s} \eta+\int_{0}^{1} \chi H(v) d t=0 .
\end{array}\right.
$$

We will show that Floer Rabinowitz homology is also independent of the function $\chi$. The reason to introduce it will become clear in the proof of vanishing result Theorem 1.2.

We can now formulate the compactness result for gradient flow lines.

Theorem 3.1. Let $H$ be a defining Hamiltonian and $\chi$ a function satisfying (6) as above. Assume that $w_{v}=\left(v_{v}, \eta_{v}\right) \in C^{\infty}\left(\mathbb{R} \times S^{1}, V\right) \times C^{\infty}(\mathbb{R}, \mathbb{R})$ for $v \in \mathbb{N}$ is a sequence of flow lines of $\nabla A^{H^{\chi}}$ for which there exists $a<b$ such that

$$
a \leq \mathscr{A}^{H^{\chi}}\left(w_{\nu}(s)\right) \leq b, \quad v \in \mathbb{N}, s \in \mathbb{R} .
$$

Then there exists a subsequence $v_{j}$ of $v$ and a flow line $w$ of $\nabla A^{H^{x}}$ such that $w_{v_{j}}$ converges in the $C_{\mathrm{loc}}^{\infty}\left(\mathbb{R} \times S^{1}, V\right) \times C_{\mathrm{loc}}^{\infty}(\mathbb{R}, \mathbb{R})$-topology to $w$.

We note that since the action is increasing along gradient flow lines it follows that if the sequence of gradient flow lines $w_{v}$ in Theorem 3.1 has fixed asymptotics $\lim _{s \rightarrow \pm \infty} w_{\nu}(s)=w^{ \pm} \in \operatorname{crit}\left(\mathscr{A}^{H^{x}}\right)$, then assertion (9) automatically holds for $a=\mathscr{A}^{H^{x}}\left(w^{-}\right)$and $b=\mathscr{A}^{H^{x}}\left(w^{+}\right)$.

The following proposition is our main tool to bound the Lagrange multiplier $\eta$.

Proposition 3.2. Let $H^{\chi}(t, x)=\chi(t) H(x)$ where $\chi$ satisfies (6) and $X_{H}=R$ along $\Sigma=H^{-1}(0)$ (but $H$ need not have compact support). Then there exist constants $\epsilon>0$ and $c<\infty$ such that the following implication holds:

$$
\left\|\nabla \mathscr{A}^{H^{x}}(v, \eta)\right\| \leq \epsilon \quad \Longrightarrow \quad|\eta| \leq c\left(\left|\mathscr{A}^{H^{\chi}}(v, \eta)\right|+1\right) .
$$


We first prove a lemma which says that the action value of a critical point of $\mathscr{A}^{H^{\chi}}$, that is, a reparametrized Reeb orbit, is given by the period.

Lemma 3.3. For $H^{\chi}$ as in Proposition 3.2 and $(v, \eta) \in \operatorname{crit}\left(\mathscr{A}^{H^{\chi}}\right)$ we have

$$
\mathscr{A}^{H^{\chi}}(v, \eta)=\eta \text {. }
$$

Proof. Inserting (7) into $\mathscr{A}^{H^{\chi}}$ and using $\alpha=\left.\lambda\right|_{\Sigma}$ we compute

$$
\mathscr{A}^{H^{\chi}}(v, \eta)=\eta \int_{0}^{1} \chi \lambda(v) R(v) d t=\eta \int_{0}^{1} \alpha(v) R(v) \chi d t=\eta \int_{0}^{1} \chi d t=\eta .
$$

Proof of Proposition 3.2. We prove the proposition in three steps. The first step is an elaboration of the observation in Lemma 3.3.

Step 1. There exists $\delta>0$ and a constant $c_{\delta}<\infty$ with the following property. For every $(v, \eta) \in \mathscr{L} \times \mathbb{R}$ such that $v(t) \in U_{\delta}=H^{-1}((-\delta, \delta))$ for every $t \in \mathbb{R} / \mathbb{Z}$, the following estimate holds:

$$
|\eta| \leq 2\left|\mathscr{A}^{H^{\chi}}(v, \eta)\right|+c_{\delta}\left\|\nabla \mathscr{A}^{H^{\chi}}(v, \eta)\right\| .
$$

Choose $\delta>0$ so small such that

$$
\lambda(x) X_{H}(x) \geq \frac{1}{2}+\delta, \quad x \in U_{\delta},
$$

and set

$$
c_{\delta}=2\left\|\left.\lambda\right|_{U_{\delta}}\right\|_{\infty}
$$

We estimate

$$
\begin{aligned}
\left|\mathscr{A}^{H^{\chi}}(v, \eta)\right| & =\left|\int_{0}^{1} \lambda(v) \partial_{t} v-\eta \int_{0}^{1} \chi H(v(t)) d t\right| \\
& =\mid \eta \int_{0}^{1} \chi \lambda(v) X_{H}(v)+\int_{0}^{1} \lambda(v)\left(\partial_{t} v-\eta \chi X_{H}(v)\right) \\
& \quad-\eta \int_{0}^{1} \chi H(v(t)) d t \mid \\
& \geq\left|\eta \int_{0}^{1} \chi \lambda(v) X_{H}(v)\right|-\left|\int_{0}^{1} \lambda(v)\left(\partial_{t} v-\chi \eta X_{H}(v)\right)\right| \\
& \geq|\eta|\left(\frac{1}{2}+\delta\right)-\frac{c_{\delta}}{2}\left\|\partial_{t} v-\chi \eta X_{H}(v)\right\|_{1}-|\eta| \delta \\
& \geq \frac{|\eta|}{2}-\frac{c_{\delta}}{2}\left\|\partial_{t} v-\chi \eta X_{H}(v)\right\|_{2} \\
& \geq \frac{|\eta|}{2}-\frac{c_{\delta}}{2}\left\|\nabla \mathscr{A}^{H^{\chi}}(v, \eta)\right\| .
\end{aligned}
$$


Step 2. For each $\delta>0$ there exists $\epsilon=\epsilon(\delta)>0$ such that if $\left\|\nabla \mathscr{A}^{H^{x}}(v, \eta)\right\| \leq \epsilon$, then $v(t) \in U_{\delta}$ for every $t \in[0,1]$.

First assume that $v \in \mathscr{L}$ has the property that there exist $t_{0}, t_{1} \in \mathbb{R} / \mathbb{Z}$ such that $\left|H\left(v\left(t_{0}\right)\right)\right| \geq \delta$ and $\left|H\left(v\left(t_{1}\right)\right)\right| \leq \delta / 2$. We claim that

$$
\left\|\nabla \mathscr{A}^{H^{\chi}}(v, \eta)\right\| \geq \frac{\delta}{2 \kappa}
$$

for every $\eta \in \mathbb{R}$, where

$$
\kappa:=\max _{x \in \bar{U}_{\delta}, t \in S^{1}}\left\|\nabla_{t} H(x)\right\|_{t} .
$$

To see this, assume without loss of generality that $t_{0}<t_{1}$ and $\delta / 2 \leq|H(v(t))| \leq \delta$ for all $t \in\left[t_{0}, t_{1}\right]$. Then we estimate

$$
\begin{aligned}
\left\|\nabla \mathscr{A}^{H^{\chi}}(v, \eta)\right\| & \geq \sqrt{\int_{0}^{1}\left\|\partial_{t} v-\chi \eta X_{H}(v)\right\|^{2} d t} \\
& \geq \int_{0}^{1}\left\|\partial_{t} v-\chi \eta X_{H}(v)\right\| d t \\
& \geq \int_{t_{0}}^{t_{1}}\left\|\partial_{t} v-\chi \eta X_{H}(v)\right\| d t \\
& \geq \frac{1}{\kappa} \int_{t_{0}}^{t_{1}}\|\nabla H(v)\| \cdot\left\|\partial_{t} v-\chi \eta X_{H}(v)\right\| d t \\
& \geq \frac{1}{\kappa} \int_{t_{0}}^{t_{1}}\left|\left\langle\nabla H(v), \partial_{t} v-\chi \eta X_{H}(v)\right\rangle\right| d t \\
& =\frac{1}{\kappa} \int_{t_{0}}^{t_{1}}\left|\left\langle\nabla H(v), \partial_{t} v\right\rangle\right| d t \\
& =\frac{1}{\kappa} \int_{t_{0}}^{t_{1}}\left|d H(v) \partial_{t} v\right| d t \\
& =\frac{1}{\kappa} \int_{t_{0}}^{t_{1}}\left|\partial_{t} H(v)\right| d t \\
& \geq \frac{1}{\kappa}\left|\int_{t_{0}}^{t_{1}} \partial_{t} H(v) d t\right| \\
& =\frac{1}{\kappa}\left|H\left(v\left(t_{1}\right)\right)-H\left(v\left(t_{0}\right)\right)\right| \\
& \geq \frac{1}{\kappa}\left(\left|H\left(v\left(t_{0}\right)\right)\right|-\left|H\left(v\left(t_{1}\right)\right)\right|\right) \\
& \geq \frac{\delta}{2 \kappa} .
\end{aligned}
$$

This proves (10). 
Now assume that $v \in \mathscr{L}$ has the property that $v(t) \in V \backslash U_{\delta / 2}$ for every $t \in[0,1]$. In this case we estimate

$$
\left\|\nabla A^{H^{\chi}}(v, \eta)\right\| \geq\left|\int_{0}^{1} \chi H(v(t)) d t\right| \geq \frac{\delta}{2}
$$

for every $\eta \in \mathbb{R}$. From (10) and (11), Step 2 follows with

$$
\epsilon=\frac{\delta}{2 \max \{1, \kappa\}} .
$$

Step 3. We prove the proposition.

Choose $\delta$ as in Step $1, \epsilon=\epsilon(\delta)$ as in Step 2 and

$$
c=\max \left\{2, c_{\delta} \epsilon\right\} .
$$

Assume that $\left\|\nabla \mathscr{A}^{H^{\chi}}(v, \eta)\right\| \leq \epsilon$. Step 1 and Step 2 imply that

$$
|\eta| \leq 2\left|\mathscr{A}^{H^{\chi}}(v, \eta)\right|+c_{\delta}\left\|\nabla \mathscr{A}^{H^{x}}(v, \eta)\right\| \leq c\left(\left|\mathscr{A}^{H^{\chi}}(v, \eta)\right|+1\right) .
$$

This proves Proposition 3.2.

A careful inspection of the proof of Proposition 3.2 shows that all the constants depend continuously on the defining Hamiltonian $H$. In particular, Proposition 3.2 can be strengthened to families.

Proposition 3.4. Assume that $H_{\sigma}$ for $0 \leq \sigma \leq 1$ is a smooth family of defining Hamiltonians for exact convex hypersurfaces $\Sigma_{\sigma}$ and $\chi_{\sigma}$ is a smooth family of functions satisfying (6). Then for $H_{\sigma}^{\chi}(t, x):=\chi_{\sigma}(t) H_{\sigma}(x)$ there exist constants $\epsilon>0$ and $c<\infty$ such that for every $0 \leq \sigma \leq 1$ the following implication holds:

$$
\left\|\nabla \mathscr{A}^{H_{\sigma}^{\chi}}(v, \eta)\right\| \leq \epsilon \quad \Longrightarrow \quad|\eta| \leq c\left(\left|\mathscr{A}^{H_{\sigma}^{\chi}}(v, \eta)\right|+1\right) .
$$

Proposition 3.2 allows us to control the size of the Lagrange multiplier $\eta$. Our first corollary considers the case of gradient flow lines.

Corollary 3.5. Assume that $w=(v, \eta) \in C^{\infty}\left(\mathbb{R} \times S^{1}, V\right) \times C^{\infty}(\mathbb{R}, \mathbb{R})$ is a gradient flow line of $\mathscr{A}^{H^{x}}$ which satisfies

$$
a \leq \mathscr{A}^{H^{\chi}}(w(s)) \leq b, \quad s \in \mathbb{R}
$$

for $a, b \in \mathbb{R}$. Then the $L^{\infty}$-norm of $\eta$ is bounded uniformly in terms of a constant $c<\infty$ which only depends on $a$ and $b$.

Proof. Let $\epsilon$ be as in Proposition 3.2. For $\sigma \in \mathbb{R}$ let $\tau(\sigma) \geq 0$ be defined by

$$
\tau(\sigma):=\inf \left\{\tau \geq 0:\left\|\nabla \mathscr{A}^{H^{\chi}}((v, \eta)(\sigma+\tau(\sigma)))\right\|<\epsilon\right\} .
$$


We abbreviate the energy of the flow line $(v, \eta)$ by

$$
\begin{aligned}
E & :=\int_{-\infty}^{\infty}\left\|\nabla \mathscr{A}^{H^{x}}(v, \eta)\right\|^{2} d s \\
& =\lim _{s \rightarrow \infty} \mathscr{A}^{H^{\chi}}(w(s))-\lim _{s \rightarrow-\infty} \mathscr{A}^{H^{x}}(w(s)) \\
& \leq b-a .
\end{aligned}
$$

We claim that

$$
\tau(\sigma) \leq \frac{b-a}{\epsilon^{2}}
$$

To see this we estimate

$$
b-a \geq \int_{\sigma}^{\sigma+\tau(\sigma)}\left\|\nabla \mathscr{A}^{H^{\chi}}(v, \eta)\right\|^{2} d s \geq \tau(\sigma) \epsilon^{2} .
$$

This implies (12).

We set

$$
M:=\max \{|a|,|b|\} .
$$

We deduce from Proposition 3.2 and the definition of $\tau(\sigma)$ that there exists a constant $c_{M}$ depending on $M$ but not on the flow line such that

$$
|\eta(\sigma+\tau(\sigma))| \leq c_{M}
$$

Set

$$
\|H\|_{\infty}=\max _{x \in V}|H(x)| .
$$

We estimate using (12), (13), and (14):

$$
\begin{aligned}
|\eta(\sigma)| & \leq|\eta(\sigma+\tau(\sigma))|+\int_{\sigma}^{\sigma+\tau(\sigma)}\left|\partial_{s} \eta(s)\right| d s \\
& =|\eta(\sigma+\tau(\sigma))|+\int_{\sigma}^{\sigma+\tau(\sigma)} \mid \int_{0}^{1} \chi H(v(s, t) d t \mid d s \\
& \leq c_{M}+\|H\|_{\infty} \tau(\sigma) \\
& \leq c_{M}+\frac{\|H\|_{\infty}(b-a)}{\epsilon^{2}} .
\end{aligned}
$$

The right hand side is independent of $\sigma$ and hence we get

$$
\|\eta\|_{\infty} \leq c_{M}+\frac{\|H\|_{\infty}(b-a)}{\epsilon^{2}} .
$$

This proves Corollary 3.5. 
Proof of Theorem 3.1. As we mentioned at the beginning of this subsection we establish compactness by proving the following uniform bounds:

- An $L^{\infty}$-bound on the loop $v \in \mathscr{L}$.

- An $L^{\infty}$-bound on the Lagrange multiplier $\eta \in \mathbb{R}$.

- An $L^{\infty}$-bound on the derivatives of the loop $v$.

Since the support of $X_{H}$ lies outside of $M \times \mathbb{R}_{+}$, the first component $v$ of a gradient flow line $(v, \eta)$ which enters $M \times \mathbb{R}_{+}$will satisfy the holomorphic curve equation by (8). By our choice of the family of almost complex structures the convexity condition guarantees that $v$ cannot touch any level set $M \times\{r\}$ from inside (see [McDuff 1991, Lemma 2.4]), and since its asymptotics lie outside of $M \times \mathbb{R}_{+}$it has to remain in the compact set $V \backslash\left(M \times \mathbb{R}_{+}\right)$all the time. This gives us a uniform $L^{\infty}$-bound on $v$. Corollary $3.5 \mathrm{implies}$ that $\eta$ remains bounded, too. Since the symplectic form $\omega$ is exact there are no nonconstant $J$-holomorphic spheres. This excludes bubbling and hence the derivatives of $v$ can be controlled; see [McDuff and Salamon 2004].

To prove invariance of Rabinowitz Floer homology under homotopies we also have to consider the case of $s$-dependent action functionals. Assume that $H_{\sigma}$ for $0 \leq \sigma \leq 1$ is a smooth family of defining Hamiltonians for exact convex hypersurfaces $\Sigma_{\sigma}$ and $\chi_{\sigma}$ is a smooth family of functions satisfying (6). To such a homotopy we associate the following two nonnegative quantities

$$
\|H\|_{\infty}=\max _{\substack{x \in V, \sigma \in[0,1]}}\left|H_{\sigma}(x)\right|, \quad\|\dot{H}\|_{\infty}=\max _{\substack{x \in V, \sigma \in[0,1]}}\left|\dot{H}_{\sigma}(x)\right|
$$

where the dot refers to the derivative with respect to the homotopy parameter $\sigma$. We further fix a smooth monotone cutoff function $\beta \in C^{\infty}(\mathbb{R},[0,1])$ satisfying $\beta(s)=1$ for $s \geq 1$ and $\beta(s)=0$ for $s \leq 0$. Using the cutoff function $\beta$ we also consider the $\mathbb{R}$-parametrized family of defining Hamiltonians

$$
H_{s}=H_{\beta(s)}, \quad s \in \mathbb{R}
$$

which is constant for $s \leq 0$ and $s \geq 1$. We set

$$
H_{-}=H_{s}, s \leq 0, \quad H_{+}=H_{s}, s \geq 1 .
$$

Accordingly we set

$$
\begin{gathered}
\chi_{s}=\chi_{\beta(s)}, s \in \mathbb{R}, \quad \chi_{-}=\chi_{s}, s \leq 0, \quad \chi_{+}=\chi_{s}, s \geq 1, \\
\|\dot{\chi}\|_{\infty}=\max _{\substack{t \in S^{1} \\
\sigma \in[0,1]}}\left|\dot{\chi}_{\sigma}(t)\right| .
\end{gathered}
$$


We abbreviate

$$
H_{s}^{\chi}=H_{s}^{\chi_{s}}, \quad H_{ \pm}^{\chi}=H_{ \pm}^{\chi_{ \pm}} .
$$

The $s$-dependent generalization of Theorem 3.1 is the following.

Theorem 3.6. Suppose that $H_{\sigma}$ for $0 \leq \sigma \leq 1$ is a smooth family of defining functions for exact convex hypersurfaces $\Sigma_{\sigma}$ and $\chi_{\sigma}$ is a smooth family of functions satisfying (6). Let $\epsilon>0$ and $c<\infty$ be constants as in Proposition 3.4. Suppose that the following inequality holds

$$
\left(c+\frac{\|H\|_{\infty}}{\epsilon^{2}}\right) \cdot\left(\|\dot{H}\|_{\infty}+\|\dot{\chi}\|_{\infty} \cdot\|H\|_{\infty}\right) \leq \frac{1}{8} .
$$

Assume that $w_{v}=\left(v_{v}, \eta_{v}\right) \in C^{\infty}\left(\mathbb{R} \times S^{1}, V\right) \times C^{\infty}(\mathbb{R}, \mathbb{R})$ for $v \in \mathbb{N}$ is a sequence of flow lines of the $s$-dependent gradient $\nabla \mathbb{A}^{H_{s}^{\chi}}$ for which there exists $a, b \in \mathbb{R}$ such that

$$
\lim _{s \rightarrow-\infty} \mathscr{A}^{H_{s}^{\chi}}\left(w_{\nu}(s)\right) \geq a, \quad \lim _{s \rightarrow \infty} \mathscr{A}^{H_{s}^{\chi}}\left(w_{\nu}(s)\right) \leq b, \quad v \in \mathbb{N}, s \in \mathbb{R} .
$$

Then there exists a subsequence $v_{j}$ of $v$ and a flow line $w$ of $\nabla \mathscr{A}^{H_{s}^{\chi}}$ such that $w_{v_{j}}$ converges in the $C_{\mathrm{loc}}^{\infty}\left(\mathbb{R} \times S^{1}, V\right) \times C_{\mathrm{loc}}^{\infty}(\mathbb{R}, \mathbb{R})$-topology to $w$.

Proof. The proof follows the same scheme as the proof of Theorem 3.1 as soon as the uniform bound on the Lagrange multiplier is established in the $s$-dependent case. This is done in Corollary 3.7 below.

Corollary 3.7. Under the assumptions of Theorem 3.6 assume that $w=(v, \eta)$ is a flow line of $\nabla \mathscr{A}^{H_{s}^{\chi}}$ such that for $a, b \in \mathbb{R}$

$$
\lim _{s \rightarrow-\infty} \mathscr{A}^{H_{s}^{\chi}}(w(s)) \geq a, \quad \lim _{s \rightarrow \infty} \mathscr{A}^{H_{s}^{\chi}}(w(s)) \leq b .
$$

Then the Lagrange multiplier $\eta$ is uniformly bounded in terms of a constant which only depends on $a$ and $b$.

Proof of Corollary 3.7. In the $s$-dependent case the action along a gradient flow line $(v, \eta)(s)$ is not necessarily increasing any more. Instead the following formula holds:

$$
\begin{aligned}
\frac{d}{d s} \mathscr{A}^{H_{s}^{\chi}}(v, \eta)(s)= & \left\|\nabla \mathscr{A}^{H_{s}^{\chi}}(v, \eta)(s)\right\|^{2}+\left(\partial_{s} \mathscr{A}^{H_{s}^{\chi}}\right)(v, \eta)(s) \\
= & \left\|\nabla \mathscr{A}^{H_{s}^{\chi}}(v, \eta)(s)\right\|^{2} \\
& -\eta(s) \int_{0}^{1}\left(\chi_{s} \frac{\partial H_{s}}{\partial s}(v)+\frac{\partial \chi_{s}}{\partial s} H_{s}(v)\right) d t \\
= & \left\|\nabla \mathscr{A}^{H_{s}^{\chi}}(v, \eta)(s)\right\|^{2} \\
& -\eta(s) \dot{\beta}(s) \int_{0}^{1}\left(\chi_{\beta(s)} \dot{H}_{\beta(s)}(v)+\dot{\chi}_{\beta(s)} H_{\beta(s)}(v)\right) d t .
\end{aligned}
$$


As in the $s$-independent case we abbreviate

$$
M:=\max \{|a|,|b|\} .
$$

Using (17) we estimate the action of the gradient flow line $(v, \eta)$ for each $s \in \mathbb{R}$ by

$$
\left|\mathscr{A}^{H_{s}^{\chi}}(v, \eta)(s)\right| \leq M+\|\eta\|_{\infty}\left(\|\dot{H}\|_{\infty}+\|\dot{\chi}\|_{\infty} \cdot\|H\|_{\infty}\right) .
$$

In the $s$-dependent case the energy is not given any more just by the action difference. If we define the energy $E$ as before we obtain

$$
\begin{aligned}
E & :=\int_{-\infty}^{\infty}\left\|\nabla \mathscr{A}^{H_{s}^{\chi}}(v, \eta)\right\|^{2} d s \\
& \leq b-a-\int_{-\infty}^{\infty}\left(\partial_{s} \mathscr{A}^{H_{s}^{\chi}}\right)(v, \eta) d s .
\end{aligned}
$$

Using (17) we obtain the estimate

$$
E \leq b-a+\|\eta\|_{\infty}\left(\|\dot{H}\|_{\infty}+\|\dot{\chi}\|_{\infty} \cdot\|H\|_{\infty}\right) .
$$

For $\epsilon>0$ as in Proposition 3.4 we define $\tau(\sigma)$ for $\sigma \in \mathbb{R}$ as in the proof of Corollary 3.5. Then the estimate (12) continues to hold. Using Proposition 3.4 as well as the estimates (12),(18), and (19) we get for any $\sigma \in \mathbb{R}$

$$
\begin{aligned}
|\eta(\sigma)| & \leq|\eta(\sigma+\tau(\sigma))|+\int_{\sigma}^{\sigma+\tau(\sigma)}\left|\partial_{s} \eta(s)\right| d s \\
& \leq c\left(\left|\mathscr{A}^{H_{\sigma+\tau(\sigma)}^{\chi}}(v, \eta)\right|+1\right)+\tau(\sigma)\|H\|_{\infty} \\
& \leq c(M+1)+c\left(\|\dot{H}\|_{\infty}+\|\dot{\chi}\|_{\infty} \cdot\|H\|_{\infty}\right)\|\eta\|_{\infty}+\frac{\|H\|_{\infty} E}{\epsilon^{2}} \\
& \leq c(M+1)+\frac{\|H\|_{\infty}(b-a)}{\epsilon^{2}} \\
& \quad+\left(c+\frac{\|H\|_{\infty}}{\epsilon^{2}}\right) \cdot\left(\|\dot{H}\|_{\infty}+\|\dot{\chi}\|_{\infty} \cdot\|H\|_{\infty}\right) \cdot\|\eta\|_{\infty} .
\end{aligned}
$$

Since $\sigma \in \mathbb{R}$ was arbitrary we obtain

$$
\begin{aligned}
\|\eta\|_{\infty} \leq c(M+1) & +\frac{\|H\|_{\infty}(b-a)}{\epsilon^{2}} \\
& +\left(c+\frac{\|H\|_{\infty}}{\epsilon^{2}}\right) \cdot\left(\|\dot{H}\|_{\infty}+\|\dot{\chi}\|_{\infty} \cdot\|H\|_{\infty}\right) \cdot\|\eta\|_{\infty} .
\end{aligned}
$$

Using the assumption (16) of the corollary we conclude that

$$
\|\eta\|_{\infty} \leq 2 c(M+1)+\frac{2\|H\|_{\infty}(b-a)}{\epsilon^{2}} .
$$


(Here a constant $1 / 2$ instead of $1 / 8$ in (16) would suffice; the smaller constant $1 / 8$ will be needed for Corollary 3.8 below). This finishes the proof of Corollary 3.7 and hence of Theorem 3.6.

We can further deduce from Corollary 3.7 the following corollary which is not needed for the proof of Theorem 3.6, but which we will need later in the invariance proof for Floer Rabinowitz homology.

Corollary 3.8. Under the assumptions of Corollary 3.7, let $\left(v^{ \pm}, \eta^{ \pm}\right)$be critical points of the Rabinowitz action functionals $\mathscr{A}^{H_{ \pm}^{x}}$ and suppose that there exists a gradient flow line $(v, \eta)(s)$ of the $s$-dependent Rabinowitz action functional $\AA^{H_{s}^{\chi}}$ between $\left(v^{-}, \eta^{-}\right)$and $\left(v^{+}, \eta^{+}\right)$.

(a) If the action at the negative asymptotic satisfies

$$
\mathscr{A}^{H} \underline{x}\left(v^{-}, \eta^{-}\right) \geq 1
$$

then the action at the positive asymptotic satisfies

$$
\mathscr{A}^{H_{+}^{\chi}}\left(v^{+}, \eta^{+}\right) \geq \frac{1}{2} \mathscr{A}^{H \underline{\chi}}\left(v^{-}, \eta^{-}\right) .
$$

(b) If the action at the positive asymptotic satisfies

$$
\mathscr{A}^{H_{+}^{\chi}}\left(v^{+}, \eta^{+}\right) \leq-1
$$

then the action at the negative asymptotic satisfies

$$
\mathscr{A}^{H} \underline{x}\left(v^{-}, \eta^{-}\right) \leq \frac{1}{2} \mathscr{A}^{H_{+}^{\chi}}\left(v^{+}, \eta^{+}\right) .
$$

Proof. We only prove cases (a) and (b) being completely analogous. We first assume that the absolute value of the action at the positive asymptotic satisfies the inequality

$$
\left|\mathscr{A}^{H_{+}^{\chi}}\left(v^{+}, \eta^{+}\right)\right| \leq \mathscr{A}^{H^{x}}\left(v^{-}, \eta^{-}\right)
$$

Using the notation from the proof of Corollary 3.7 we deduce from (23) that

$$
M=\mathscr{A}^{H-\underline{x}}\left(v^{-}, \eta^{-}\right), \quad b-a \leq 0 .
$$

Hence we get from (20) and (21) the inequalities

$$
\|\eta\|_{\infty} \leq 2 c\left(\mathscr{A}^{H \underline{x}}\left(v^{-}, \eta^{-}\right)+1\right) \leq 4 c \mathscr{A}^{H \underline{x}}\left(v^{-}, \eta^{-}\right) .
$$


This implies together with (16) and (19)

$$
\begin{aligned}
\mathscr{A}^{H_{+}^{\chi}}\left(v^{+}, \eta^{+}\right) & \geq \mathscr{A}^{H \underline{x}}\left(v^{-}, \eta^{-}\right)-\|\eta\|_{\infty}\left(\|\dot{H}\|_{\infty}+\|\dot{\chi}\|_{\infty} \cdot\|H\|_{\infty}\right) \\
& \geq\left(1-4 c\left(\|\dot{H}\|_{\infty}+\|\dot{\chi}\|_{\infty} \cdot\|H\|_{\infty}\right)\right) \mathscr{A}^{H^{-}}\left(v^{-}, \eta^{-}\right) \\
& \geq \frac{1}{2} \mathscr{A}^{H^{-}}\left(v^{-}, \eta^{-}\right) .
\end{aligned}
$$

This implies the corollary under the additional assumption (23).

Now assume that (23) does not hold. To prove the corollary it suffices to exclude the case

$$
\mathscr{A}^{H_{+}^{\chi}}\left(v^{+}, \eta^{+}\right)<-\mathscr{A}^{H^{\alpha}}\left(v^{-}, \eta^{-}\right) .
$$

We assume by contradiction (24). We then obtain

$$
M=-\mathscr{A}^{H_{+}^{\chi}}\left(v^{+}, \eta^{+}\right), \quad b-a \leq 0 .
$$

In particular, from (20) and (21) we get

$$
\|\eta\|_{\infty} \leq-4 c \mathscr{A}^{H_{+}^{\chi}}\left(v^{+}, \eta^{+}\right) .
$$

Hence

$$
\begin{aligned}
\mathscr{A}^{H^{\underline{\chi}}}\left(v^{-}, \eta^{-}\right) & \leq \mathscr{A}^{H_{+}^{\chi}}\left(v^{+}, \eta^{+}\right)+\|\eta\|_{\infty}\left(\|\dot{H}\|_{\infty}+\|\dot{\chi}\|_{\infty} \cdot\|H\|_{\infty}\right) \\
& \leq\left(1-4 c\left(\|\dot{H}\|_{\infty}+\|\dot{\chi}\|_{\infty} \cdot\|H\|_{\infty}\right)\right) \mathscr{A}_{+}^{H_{+}^{\chi}}\left(v^{+}, \eta^{+}\right) \\
& \leq \frac{1}{2} \mathscr{A}^{H_{+}^{\chi}}\left(v^{+}, \eta^{+}\right) \\
& <-\frac{1}{2} \mathscr{A}^{H^{-}}\left(v^{-}, \eta^{-}\right) .
\end{aligned}
$$

But this implies that

$$
\mathscr{A}^{H} \underline{x}\left(v^{-}, \eta^{-}\right)<0
$$

which contradicts assumption (21). Hence (24) has to be wrong and Corollary 3.8 follows.

\subsection{Proof of Theorem 1.1.}

Definition of Rabinowitz Floer homology. The Rabinowitz action functional $\mathscr{A}^{H}$ is invariant under the $S^{1}$-action on $\mathscr{L} \times \mathbb{R}$ given by $t_{*}(v(\cdot), \eta) \mapsto(v(t+\cdot), \eta)$. In particular, the action functional $\mathscr{A}^{H}$ is never Morse. Similar things happen for the perturbed version $\mathscr{A}^{H^{\chi}}$ since $\mathscr{A}^{H^{\chi}}$ is still invariant under a reparametrized action of the circle on $\mathscr{L} \times \mathbb{R}$. However, generically $\mathscr{A}^{H^{\chi}}$ is Morse-Bott, simultaneously for all perturbations $\chi$. We define Rabinowitz Floer homology in this subsection under the Morse-Bott assumption and then use invariance established in the next 
subsection which allows us to perturb the hypersurface to make the Rabinowitz action functional Morse-Bott.

More precisely, we make the following nondegeneracy assumption on the Reeb flow $\phi_{t}$ of the contact form $\alpha$ on $\Sigma$.

(A) The closed Reeb orbits of $(\Sigma, \alpha)$ are of Morse-Bott type, that is, for each $T \in \mathbb{R}$ the set $\mathcal{N}_{T} \subset \Sigma$ formed by the $T$-periodic Reeb orbits is a closed submanifold, the rank of $\left.d \alpha\right|_{\mathcal{N}_{T}}$ is locally constant, and $T_{p} \mathcal{N}_{T}=\operatorname{ker}\left(T_{p} \phi_{T}-\right.$ id) for all $p \in \mathcal{N}_{T}$.

Assumption (A) is satisfied for generic hypersurfaces. (This result is of course well known, but since we did not find a proof in the literature we include one in Appendix B). Note that the contact condition is an open condition and assumption (A) is generically satisfied. Since we prove that our homology is invariant under homotopies we can assume without loss of generality that (A) holds. If (A) is satisfied, then the action functional $\mathscr{A}^{H^{x}}$ is Morse-Bott.

Remark. In fact, we prove in Appendix B that on generic hypersurfaces all $T$ periodic Reeb orbits $\gamma$ with $T \neq 0$ are nondegenerate, that is, the linearization $T_{p} \phi_{T}: \xi_{p} \rightarrow \xi_{p}$ at $p=\gamma(0)$ does not have 1 in its spectrum. In this case the critical manifold of $\mathscr{A}^{H^{\chi}}$ consists of a union of circles (corresponding to $k$-fold covers for $0 \neq k \in \mathbb{Z}$ ) for each simple nonconstant Reeb orbit and a copy of the hypersurface $\Sigma$ for the constant solutions, that is, critical points with $\eta=0$.

There are several ways to deal with Morse-Bott situations in Floer homology. One possibility is to choose an additional small perturbation to get a Morse situation. This was carried out by Pozniak [1999], where it was also shown that the local Floer homology near each critical manifold coincides with the Morse homology of the critical manifold. (In fact, the construction in [Poźniak 1999] is for Lagrangian intersections; see [Biran et al. 2003] for the transfer to the periodic orbit case). Another possibility is to choose an additional Morse function on the critical manifold. The chain complex is then generated by the critical points of this Morse function while the boundary operator is defined by counting flow lines with cascades. This approach was carried out by the second named author in [2004] and we recall it in Appendix A. And though it is formulated in finite dimensions, it directly carries over to the case of Floer homology; see also [Bourgeois 2003] and [Bourgeois and Oancea 2007] for discussions of Morse-Bott homology in infinite dimensional situations.

Following the second approach we choose an additional Morse function $h$ on $\operatorname{crit}\left(\mathscr{A}^{H^{\chi}}\right)$. The Floer chain group $C F\left(\mathscr{A}^{H^{\chi}}, h\right)$ is defined as the $\mathbb{Z}_{2}$-vector space 
consisting of formal sums of critical points of $h$

$$
x=\sum_{c \in \operatorname{crit}(h)} x_{c} c
$$

where the coefficients $x_{c} \in \mathbb{Z}_{2}$ satisfy the finiteness condition

$$
\#\left\{c \in \operatorname{crit}(h): x_{c} \neq 0, \mathscr{A}^{H^{\chi}}(c) \geq \kappa\right\}<\infty
$$

for every $\kappa \in \mathbb{R}$. To define the boundary operator, we choose an additional Riemannian metric $g$ on the critical manifold $\operatorname{crit}\left(\mathscr{A}^{H^{x}}\right)$. For two critical points $c_{-}, c_{+} \in \operatorname{crit}(h)$ we consider the moduli space of gradient flow lines with cascades $\mu_{c_{-}, c_{+}}\left(\mathscr{A}^{H^{\chi}}, h, J, g\right)$; see Appendix A. For a generic choice of $J$ and $g$ this moduli space of is a smooth manifold. It follows from the Compactness Theorem 3.1 that its zero dimensional component $\mu_{c_{-}, c_{+}}^{0}\left(\mathscr{A}^{H^{\chi}}, h, J, g\right)$ is actually compact and hence a finite set.

We now set

$$
n\left(c_{-}, c_{+}\right)=\# \mathcal{M}_{c_{-}, c_{+}}^{0}\left(\mathscr{A}^{H^{\chi}}, h, J, g\right) \bmod 2 \in \mathbb{Z}_{2}
$$

and define the Floer boundary operator

$$
\text { д: } C F\left(\mathscr{A}^{H^{\chi}}, h\right) \rightarrow C F\left(\mathscr{A}^{H^{\chi}}, h\right)
$$

as the linear extension of

$$
\partial c:=\sum_{c^{\prime} \in \operatorname{crit}(h)} n\left(c^{\prime}, c\right) c^{\prime}
$$

for $c \in \operatorname{crit}(h)$. Note the reversed order of the arguments in $n\left(c^{\prime}, c\right)$, which reflects the fact that we define homology rather than cohomology.

For this definition to make sense, we need to verify that the sum in the definition of $\partial c$ satisfies the finiteness condition (25) for every $c$. Since $n\left(c^{\prime}, c\right) \neq 0$ implies $\mathscr{A}^{H^{x}}\left(c^{\prime}\right) \leq \mathscr{A}^{H^{x}}(c)$, it suffices to show

$$
\#\left\{c \in \operatorname{crit}(h): a \leq \mathscr{A}^{H^{\chi}}(c) \leq b\right\}<\infty
$$

for all $a<b$. Since by Lemma 3.3 the action $\mathscr{A}^{H^{x}}$ at a critical point is given by the period, this finiteness statement follows from the Arzela-Ascoli theorem.

Again using the Compactness Theorem 3.1 the standard argument in Floer theory shows that

$$
\partial^{2}=0
$$

We define the Floer homology as usual by

$$
H F\left(\mathscr{A}^{H^{\chi}}, h, J, g\right)=\frac{\operatorname{ker} \partial}{\operatorname{im} \partial} .
$$


Standard arguments show that $\operatorname{HF}\left(\mathscr{A}^{H^{\chi}}, h, J, g\right)$ is independent up to canonical isomorphism of the choices of $h, J$ and $g$, so we will denote it simply by $H F\left(\mathscr{A}^{H^{\chi}}\right)$.

Invariance. It remains to prove invariance of $H F\left(\mathscr{A}^{H^{x}}\right)$ under homotopies of the Hamiltonians $H^{\chi}$. So consider a smooth family of defining Hamiltonians $H_{\sigma}$ for exact convex hypersurfaces $\Sigma_{\sigma}$ and a smooth family of functions $\chi_{\sigma}$ satisfying (6). Let $\epsilon>0$ and $c<\infty$ be the constants for the homotopy $H_{\sigma}$ in Proposition 3.4. Fix $N \in \mathbb{N}$ and define for $0 \leq j \leq N-1$ the homotopies

$$
H_{\sigma}^{j}=H_{j+\sigma / N}, \quad \chi_{\sigma}^{j}=\chi_{j+\sigma / N} .
$$

By choosing $N \in \mathbb{N}$ large enough we can assume that for each $0 \leq j \leq N-1$ the inequality

$$
\left(c+\frac{\left\|H^{j}\right\|_{\infty}}{\epsilon^{2}}\right) \cdot\left(\left\|\dot{H}^{j}\right\|_{\infty}+\left\|\dot{\chi}^{j}\right\|_{\infty} \cdot\left\|H^{j}\right\|_{\infty}\right) \leq \frac{1}{16} .
$$

holds. To simplify notation, we replace the original homotopy by the homotopy $\left(H_{\sigma}^{j}, \chi_{\sigma}^{j}\right)$ for some $j$ and drop the index $j$, so that $H_{\sigma}$ satisfies inequality (27).

Define the $(s, t)$-dependent Hamiltonian $H_{s}^{\chi}$ and $H_{ \pm}^{\chi}$ as in the discussion before Theorem 3.6 and pick Morse functions $h^{ \pm}$on the $\operatorname{crit}\left(\mathscr{A}_{ \pm}^{H_{ \pm}^{\chi}}\right)$. For two critical points $c_{ \pm} \in \operatorname{crit}\left(h^{ \pm}\right)$we consider the moduli space of gradient flow lines with cascades $\mu_{c_{-}, c_{+}}\left(\mathscr{A}^{H_{s}^{\chi}}, h, J, g^{ \pm}\right)$for the $s$-dependent action functional $\mathscr{A}^{H_{s}^{\chi}}$. It follows from the Compactness Theorem 3.6 that its zero dimensional component $\mu_{c_{-}, c_{+}}^{0}\left(\mathscr{A}^{H_{s}^{\chi}}, h, J, g^{ \pm}\right)$is a finite set. We set

$$
m\left(c_{-}, c_{+}\right)=\# \mu_{c_{-}, c_{+}}^{0}\left(\mathscr{A}^{H_{s}^{\chi}}, h, J, g^{ \pm}\right) \bmod 2 \in \mathbb{Z}_{2}
$$

and define a linear map

$$
\phi: C F\left(\mathscr{A}^{H_{+}^{\chi}}, h^{+}\right) \rightarrow C F\left(\mathscr{A}^{H \underline{\chi}}, h_{-}\right)
$$

as the linear extension of

$$
\phi\left(c_{+}\right):=\sum_{c_{-} \in \operatorname{crit}\left(h^{-}\right)} m\left(c_{-}, c_{+}\right) c_{-}
$$

for $c_{+} \in \operatorname{crit}\left(h^{+}\right)$.

Again, we need to verify that the sum in the definition of $\phi\left(c_{+}\right)$satisfies the finiteness condition (25) for every $c_{+}$. Since by Corollary 3.8, $m\left(c_{-}, c_{+}\right) \neq 0$ implies $\mathscr{A}^{H-x}\left(c_{-}\right) \leq \max \left(2 \mathscr{A}^{H_{+}^{\chi}}\left(c_{+}\right), 1\right)$, this follows again from the finiteness statement (26) for $H \underline{\chi}$. 
Again using the Compactness Theorem 3.6 the standard argument in Floer theory shows that

$$
\partial^{-} \phi=\phi \partial^{+}
$$

so $\phi$ induces a map on Floer homology

$$
\Phi: H F\left(\mathscr{A}^{H_{+}^{\chi}}\right) \rightarrow H F\left(\mathscr{A}^{H} \underline{x}\right) .
$$

For $\sigma \in[0,1]$ define the inverse homotopy

$$
\bar{H}_{\sigma}=H_{1-\sigma}, \quad \bar{\chi}_{\sigma}=\chi_{1-\sigma} .
$$

Again this homotopy yields a homomorphism

$$
\Psi: H F\left(\mathscr{A}^{H \underline{x}}\right) \rightarrow H F\left(\mathscr{A}^{H_{+}^{\chi}}\right)
$$

by counting gradient flow lines of $\mathscr{A}^{\bar{H}_{s}^{\bar{x}}}$. For $R \geq 1$ define the concatenation of $H_{S}^{\chi}$ and $\bar{H}_{s}^{\bar{\chi}}$ by the formula

$$
K_{s}^{\chi}=H_{s}^{\chi} \#_{R} \bar{H}_{s}^{\bar{\chi}}= \begin{cases}H_{s+R}^{\chi} & s \leq 0 \\ \bar{H}_{s-R}^{\bar{\chi}} & s \geq 0 .\end{cases}
$$

The $s$-dependent Hamiltonian $K_{s}^{\chi}$ gives a homotopy from $H_{-}^{\chi}$ via $H_{+}^{\chi}$ back to $H_{-}$. From (27) it follows that

$$
\left(c+\frac{\|K\|_{\infty}}{\epsilon^{2}}\right) \cdot\left(\|\dot{K}\|_{\infty}+\left\|\dot{\chi^{j}}\right\|_{\infty} \cdot\|K\|_{\infty}\right) \leq \frac{1}{8} .
$$

Using again Corollary 3.7 and Corollary 3.8 together with the standard gluing argument we see that the composition

$$
\Phi \circ \Psi: H F\left(\mathscr{A}^{H \underline{x}}\right) \rightarrow H F\left(\mathscr{A}^{H \underline{x}}\right)
$$

is given by counting gradient flow lines of $\mathscr{A}^{K_{s}^{\chi}}$. Now for $r \in[0,1]$ consider the homotopy of homotopies

$$
H_{s}^{\chi, r}=H_{r s}^{\chi}, \quad \bar{H}_{s}^{\bar{\chi}, r}=\bar{H}_{r s}^{\bar{\chi}} .
$$

Define further for $s \in \mathbb{R}$

$$
K_{s}^{\chi, r}=H_{s}^{\chi, r} \#_{R} \bar{H}_{S}^{\bar{\chi}, r} .
$$

Then for each $r \in[0,1]$ the estimate

$$
\left(c+\frac{\left\|K^{r}\right\|_{\infty}}{\epsilon^{2}}\right) \cdot\left(\left\|\dot{K}^{r}\right\|_{\infty}+\|\dot{\chi}\|_{\infty} \cdot\left\|K^{r}\right\|_{\infty}\right) \leq \frac{1}{8} .
$$

continues to hold. Moreover,

$$
K_{s}^{\chi, 0}=H_{-}^{\chi}
$$


does not depend on the $s$-variable any more and therefore induces the identity on Floer homology. It follows that

$$
\Phi \circ \Psi=\mathrm{id}: H F\left(\mathscr{A}^{H \underline{x}}\right) \rightarrow H F\left(\mathscr{A}^{H \underline{x}}\right) .
$$

Similarly, by concatenating $\bar{H}_{s}^{\bar{\chi}}$ with $H_{s}^{\chi}$ we see that

$$
\Psi \circ \Phi=\mathrm{id}: H F\left(\mathscr{A}^{H_{+}^{\chi}}\right) \rightarrow H F\left(\mathscr{A}^{H_{+}^{\chi}}\right),
$$

hence $\Phi$ defines an isomorphism between the Floer homology groups $H F\left(\mathscr{A}^{H_{+}^{x}}\right)$ and $H F\left(\mathscr{A}^{H-\underline{x}}\right)$ with inverse $\Psi$. This finishes the proof of Theorem 1.1.

3.3. Proof of Theorem 1.2. For the proof of Theorem 1.2 we will consider more general Rabinowitz action functionals depending on two (possibly time-dependent) Hamiltonians $H, F \in C^{\infty}(\mathbb{R} / \mathbb{Z} \times V)$,

$$
\mathscr{A}_{F}^{H}(v, \eta):=\mathscr{A}^{H}(v, \eta)-\int_{0}^{1} F(t,(v(t)) d t .
$$

Critical points $(v, \eta) \in \mathscr{L} \times \mathbb{R}$ of $\mathscr{A}_{F}^{H}$ are solutions of the equation

$$
\left\{\begin{array}{c}
\partial_{t} v=\eta X_{H}(v)+X_{F}(v) \\
\int_{0}^{1} H(t, v(t)) d t=0
\end{array}\right.
$$

The gradient of $\mathscr{A}_{F}^{H}$ with respect to the $L^{2}$-metric on $\mathscr{L} \times \mathbb{R}$ induced by a family of almost complex structures $J_{t}$ is given by the formula

$$
\nabla \mathscr{A}_{F}^{H}(v, \eta)=\left(\begin{array}{c}
J(t, v)\left(\partial_{t} v-\eta X_{H}(t, v)-X_{F}(t, v)\right) \\
-\int_{0}^{1} H(t, v) d t
\end{array}\right) .
$$

Next consider $s$ - and $t$-dependent Hamiltonians $H, F \in C^{\infty}(\mathbb{R} \times \mathbb{R} / \mathbb{Z} \times V)$ which agree with $s$-independent Hamiltonians $H^{ \pm}, F^{ \pm} \in C^{\infty}(\mathbb{R} / \mathbb{Z} \times V)$ for large $\pm s$. Then gradient flow lines of $\nabla \mathscr{A}_{F}^{H}$ with respect to an $s$ - and $t$-dependent almost complex structure $J$ are solutions $(v, \eta) \in C^{\infty}\left(\mathbb{R} \times S^{1}, V \times \mathbb{R}\right)$ of the following problem

$$
\left\{\begin{array}{c}
\partial_{s} v+J(s, t, v)\left(\partial_{t} v-\eta X_{H}(s, t, v)-X_{F}(s, t, v)\right)=0 \\
\partial_{s} \eta+\int_{0}^{1} H(s, t, v) d t=0 .
\end{array}\right.
$$

In particular, if $X_{H}, X_{F}$ vanish outside a compact set $K \subset V$ for all $(s, t)$ the first equation shows that $v$ is $J$-holomorphic outside $K$. If in addition $J$ is cylindrical outside $K$ the maximum principle shows that $v$ cannot exit $K$. 
In the $s$-dependent case the action along a gradient flow line $w=(v, \eta)(s)$ is not necessarily decreasing any more. Instead of that the following formula holds:

$$
\begin{aligned}
\frac{d}{d s} \mathscr{A}_{F}^{H}(v, \eta)(s) & =\left\|\nabla \mathscr{A}_{F}^{H}(v, \eta)(s)\right\|^{2}+\left(\partial_{s} \mathscr{A}_{F}^{H}\right)(v, \eta)(s) \\
= & \left\|\nabla \mathscr{A}_{F}^{H}(v, \eta)(s)\right\|^{2}-\eta(s) \int_{0}^{1} \frac{\partial H}{\partial s}(s, t, v) d t \\
& -\int_{0}^{1} \frac{\partial F}{\partial s}(s, t, v) d t \\
\geq\left\|\nabla \mathscr{A}_{F}^{H}(v, \eta)(s)\right\|^{2}-\|\eta\|_{\infty} \int_{0}^{1} \max _{x} \frac{\partial H}{\partial s}(s, t, x) d t & -\int_{0}^{1} \max _{x} \frac{\partial F}{\partial s}(s, t, x) d t .
\end{aligned}
$$

We set

$$
\left\|\partial_{s} H\right\|_{1, \infty}:=\int_{-\infty}^{\infty} \int_{0}^{1} \max _{x} \frac{\partial H}{\partial s}(s, t, x) d t d s,
$$

and similarly for $F$. Moreover, we abbreviate

$$
M:=\max \left\{\left|\mathscr{A}_{F_{+}}^{H_{+}}\left(v^{+}, \eta^{+}\right)\right|,\left|\mathscr{A}_{F_{-}}^{H_{-}}\left(v^{-}, \eta^{-}\right)\right|\right\},
$$

Using (31) we estimate the action of the gradient flow line $(v, \eta)$ for each $s \in \mathbb{R}$ by

$$
\left|\mathscr{A}_{F}^{H}(v, \eta)(s)\right| \leq M+\|\eta\|_{\infty}\left\|\partial_{s} H\right\|_{1, \infty}+\left\|\partial_{s} F\right\|_{1, \infty} .
$$

In the $s$-dependent case the energy is not given any more just by the action difference. We abbreviate by

$$
\Delta:=\mathscr{A}_{F_{+}}^{H_{+}}\left(v^{+}, \eta^{+}\right)-\mathscr{A}_{F_{-}}^{H_{-}}\left(v^{-}, \eta^{-}\right)
$$

the action difference. Then the energy is given by

$$
\begin{aligned}
E & :=\int_{-\infty}^{\infty}\left\|\nabla \mathscr{A}_{F}^{H}(v, \eta)(s)\right\|^{2} d s \\
& =\Delta-\int_{-\infty}^{\infty}\left(\partial_{s} \mathscr{A}_{F}^{H}\right)(v, \eta)(s) d s .
\end{aligned}
$$

Using (31) we obtain the estimate

$$
E \leq \Delta+\|\eta\|_{\infty}\left\|\partial_{s} H\right\|_{1, \infty}+\left\|\partial_{s} F\right\|_{1, \infty} .
$$

Proof of Theorem 1.2. For a defining Hamiltonian $H$ we abbreviate the support of its Hamiltonian vector field by

$$
\mathfrak{S}\left(X_{H}\right):=\operatorname{cl}\left\{x \in V: X_{H}(x) \neq 0\right\} .
$$


If $F \in C^{\infty}(\mathbb{R} / \mathbb{Z} \times V)$ is a displacing Hamiltonian for $\Sigma$ we can choose a defining Hamiltonian in such a way that

$$
\phi_{F}^{1}\left(\mathfrak{S}\left(X_{H}\right)\right) \cap \mathfrak{S}\left(X_{H}\right)=\varnothing .
$$

We first consider the following version of the Rabinowitz action functional $\mathscr{A}^{H}$. Pick a smooth function $\chi \in C^{\infty}\left(S^{1}, \mathbb{R}\right)$ with the following properties

$$
\operatorname{supp}(\chi) \subset(0,1 / 2), \quad \int_{0}^{1} \chi(t) d t=1 .
$$

We define the time-dependent Hamiltonian $H^{\chi}(t, x)=\chi(t) H(x)$ as before and consider the following perturbation of $\mathscr{A}^{H^{\chi}}$. We first modify the displacing Hamiltonian $F$ in the following way. Choose a smooth map $\rho \in C^{\infty}([0,1],[0,1])$ satisfying

For $t \in \mathbb{R}$ set

$$
\left.\rho\right|_{[0,1 / 2]}=0, \quad \rho(1)=1 .
$$

Note that

$$
F_{t}^{\rho}:=\dot{\rho}(t) F_{\rho(t)} \in C^{\infty}(V)
$$

We define

$$
\phi_{F^{\rho}}^{t}=\phi_{F}^{\rho(t)}
$$

$$
\mathscr{A}_{F^{\rho}}^{H^{\chi}}: \mathscr{L} \times \mathbb{R} \rightarrow \mathbb{R}
$$

by

$$
\mathscr{A}_{F^{\rho}}^{H^{\chi}}(v, \eta):=\mathscr{A}^{H^{\chi}}(v, \eta)-\int_{0}^{1} F_{t}^{\rho}((v(t)) d t .
$$

Critical points $(v, \eta) \in \mathscr{L} \times \mathbb{R}$ of $\mathscr{A}_{F^{\rho}}^{H^{\chi}}$ are solutions of the equation

$$
\left\{\begin{array}{c}
\partial_{t} v=\eta \chi X_{H}(v)+\dot{\rho} X_{F}(v) \\
\int_{0}^{1} \chi(t) H(v(t)) d t=0
\end{array}\right.
$$

It is easy to check that there are no solutions of (35). Indeed, assume first that $(v, \eta)$ is a solution of (35) with

$$
v(0) \notin \mathfrak{S}\left(X_{H}\right) .
$$

Since $\dot{\rho}$ has support in $(1 / 2,1)$ it follows from the first equation in (35) that

$$
\left.v\right|_{[0,1 / 2]} \equiv v(0) \notin \mathfrak{S}\left(X_{H}\right) .
$$

Since 0 is a regular value of $H$ it follows that

$$
\left.H(v)\right|_{[0,1 / 2]} \equiv c \neq 0 .
$$


In particular, since $\chi$ has support in $(0,1 / 2)$ we obtain

$$
\int_{0}^{1} \chi(t) H(v(t)) d t=c \int_{0}^{1 / 2} \chi(t) d t=c \neq 0
$$

contradicting the second equation in (35). So assume that $(v, \eta)$ is a solution of (35) such that

$$
v(0) \in \mathfrak{S}\left(X_{H}\right) .
$$

Since the vector fields $X_{H^{x}}$ and $X_{F^{\circ}}$ are supported in disjoint time intervals we obtain from the first equation in (35) that

$$
v(0)=v(1)=\phi_{F^{\rho}}^{1} \circ \phi_{\eta H^{\chi}}^{1}(v(0))=\phi_{F}^{1} \circ \phi_{H}^{\eta}(v(0)) .
$$

Since $v(0) \in \mathfrak{S}\left(X_{H}\right)$ we conclude that $\phi_{H}^{\eta}\left((v(0)) \in \mathfrak{S}\left(X_{H}\right)\right.$, and since $\phi_{F}$ disjoins $\mathfrak{S}\left(X_{H}\right)$ from itself we conclude with (37) that $v(0) \notin \mathfrak{S}\left(X_{H}\right)$ contradicting (36). This proves that

$$
\operatorname{crit}\left(\mathscr{A}_{F^{\rho}}^{H^{\chi}}\right)=\varnothing
$$

Remark. At this point, one would like to interpret (38) as vanishing of the Floer homology $H F\left(\mathscr{A}_{F^{\rho}}^{H^{\chi}}\right)$ and conclude the proof of Theorem 1.2 by showing the isomorphism $H F\left(\mathscr{A}^{H^{\chi}}\right) \cong H F\left(\mathscr{A}_{F^{\rho}}^{H^{\chi}}\right)$. This would require a definition of the Floer homology $H F\left(\mathscr{A}_{F^{\rho}}^{H^{\chi}}\right)$ for arbitrary $H, F$ and can indeed be done. However, below we will prove $H F\left(\mathscr{A}^{H^{\chi}}\right)=0$ more directly without actually defining the more general Floer homologies $H F\left(\mathscr{A}_{F^{\circ}}^{H^{\chi}}\right)$.

For the proof of Theorem 1.2 we need the following lemma which strengthens the assertion (38).

Lemma 3.9. Let $H^{\chi}$ and $F^{\rho}$ be as above. Let $J_{t}$ be a smooth family of $\omega$ compatible almost complex structures on $V$. Then there exists a constant $\gamma=$ $\gamma(J)>0$ such that for any $(v, \eta) \in \mathscr{L} \times \mathbb{R}$

$$
\left\|\nabla_{J} \mathscr{A}_{F^{\rho}}^{H^{\chi}}(v, \eta)\right\|_{J} \geq \gamma .
$$

Proof. Recall that for $(v, \eta) \in \mathscr{L} \times \mathbb{R}$ the gradient of the perturbed action functional $\mathscr{A}_{F^{\rho}}^{H^{\chi}}$ with respect to the metric on $\mathscr{L} \times \mathbb{R}$ induced from the family of almost complex structures $J_{t}$ is given by the formula

$$
\nabla \mathscr{A}_{F^{\rho}}^{H^{\chi}}(v, \eta)=\left(\begin{array}{c}
J_{t}(v)\left(\partial_{t} v-\eta \chi X_{H}(v)-\dot{\rho} X_{F}(v)\right) \\
-\int_{0}^{1} \chi(t) H(v(t)) d t
\end{array}\right) .
$$


We prove the lemma in three steps.

Step 1. There exists $\epsilon_{0}=\epsilon_{0}(J)$ having the following property. Assume that $(v, \eta) \in$ $\mathscr{L} \times \mathbb{R}$ satisfies

$$
\left\|\partial_{t} v-\eta \chi X_{H}(v)-\dot{\rho} X_{F_{\rho}}(v)\right\|_{2}<\epsilon_{0} .
$$

Then

$$
(v(0), v(1 / 2)) \notin \mathfrak{S}\left(X_{H}\right) \times \mathfrak{S}\left(X_{H}\right) .
$$

For $x \in V$ define a norm on the tangent space $T_{x} V$ by

$$
\|\hat{x}\|_{J, F, \rho}=\min _{t \in[0,1]}\left\|d \phi_{F}^{\rho(t)}(x) \hat{x}\right\|_{J_{t}}, \quad \hat{x} \in T_{x} V .
$$

Let

$$
d_{J, F, \rho}^{1}: V \times V \rightarrow[0, \infty)
$$

be the metric on $V$ induced from the norm $\|\cdot\|_{J, F, \rho}$. Define a second metric

$$
d_{J, F, \rho}^{2}=\left(\phi_{F}^{1}\right)_{*} d_{J, F, \rho}: V \times V \rightarrow[0, \infty) .
$$

Since $\mathfrak{S}\left(X_{H}\right)$ is compact and $\phi_{F}^{1}$ displaces $\mathfrak{S}\left(X_{H}\right)$ from itself there exists $\epsilon_{0}>0$ such that

$$
d_{J, F, \rho}^{2}\left(\mathfrak{S}\left(X_{H}\right), \phi_{F}^{1}\left(\mathfrak{S}\left(X_{H}\right)\right)\right)=\epsilon_{0}
$$

Now suppose that $(v, \eta)$ satisfies the assumptions of Step 1 We argue by contradiction and assume that

$$
v(0) \in \mathfrak{S}\left(X_{H}\right), \quad v(1 / 2) \in \mathfrak{S}\left(X_{H}\right)
$$

Let us denote $\phi_{F}^{-s}:=\left(\phi_{F}^{s}\right)^{-1}$ for $s \in[0,1]$ and define a path $w \in C^{\infty}([0,1], V)$ by

$$
w(t):=\phi_{F}^{-\rho((t+1) / 2)}(v((t+1) / 2)) .
$$

Solving for $v((t+1) / 2)$ and differentiating we find

$$
\partial_{t} w=\frac{1}{2} d \phi_{F}^{-\rho}(v)\left(\partial_{t} v-\dot{\rho} X_{F_{\rho}}(v)\right) .
$$

By (41) the endpoints of this path satisfy

$$
w(0)=v(1 / 2) \in \mathfrak{S}\left(X_{H}\right), \quad w(1)=\phi_{F}^{-1}(v(1))=\phi_{F}^{-1}(v(0)) \in \phi_{F}^{-1}\left(\mathfrak{S}\left(X_{H}\right)\right) .
$$


Using (42), the definitions of $\epsilon_{0}$ and the metric $d^{2}$ as well as the fact that $\chi$ vanishes on the interval $[1 / 2,1]$ we deduce that

$$
\begin{aligned}
\epsilon_{0} & \leq d_{J, F, \rho}^{2}\left(\phi_{F}^{1}(w(0)), \phi_{F}^{1}(w(1))\right) \\
& =d_{J, F, \rho}^{1}(w(0), w(1)) \\
& \leq \int_{0}^{1}\left\|\partial_{t} w\right\|_{J, F, \rho} d t \\
& =\int_{1 / 2}^{1}\left\|d \phi_{F}^{-\rho}(v)\left(\partial_{t} v-\dot{\rho} X_{F_{\rho}}(v)\right)\right\|_{J, F, \rho} d t \\
& \leq \int_{1 / 2}^{1}\left\|\partial_{t} v-\dot{\rho} X_{F_{\rho}}(v)\right\|_{J_{t}} d t \\
& \leq\left\|\partial_{t} v-\eta \chi X_{H}(v)-\dot{\rho} X_{F_{\rho}}(v)\right\|_{1} \\
& \leq\left\|\partial_{t} v--\eta \chi X_{H}(v) \dot{\rho} X_{F_{\rho}}(v)\right\|_{2} \\
& <\epsilon_{0} .
\end{aligned}
$$

This contradiction proves Step 1.

Step 2. There exist $\epsilon_{1}>0$ and $\delta>0$ with the following property. Assume that

$$
\left\|\partial_{t} v-\eta \chi X_{H}(v)-\dot{\rho} X_{F_{\rho}}(v)\right\|_{2}<\epsilon_{1} .
$$

Then

$$
|H(v(t))| \geq \frac{\delta}{2} \quad \text { for all } t \in[0,1 / 2] .
$$

Since $H$ is constant outside of a compact set and 0 is a regular value of $H$ we conclude that

$$
\inf _{V \backslash \mathfrak{S}\left(X_{H}\right)}|H|=\delta>0 .
$$

It follows from the first estimate in Step 2 of Proposition 3.2 that there exists $\epsilon_{0}^{\prime}>0$ such that if

$$
\left\|\partial_{t} v-\eta \chi X_{H}(v)-\dot{\rho} X_{F_{\rho}}(v)\right\|_{2}<\epsilon_{0}^{\prime}
$$

then

$$
\max _{\left(t_{0}, t_{1}\right) \in[0,1 / 2]^{2}}\left|H\left(v\left(t_{0}\right)\right)-H\left(v\left(t_{1}\right)\right)\right| \leq \frac{\delta}{2} .
$$

Here we have used $\rho=0$ on $[0,1 / 2]$. Let $\epsilon_{0}$ be as in Step 1 and set

$$
\epsilon_{1}=\min \left\{\epsilon_{0}, \epsilon_{0}^{\prime}\right\}
$$

Then it follows from Step 1 that

$$
\max _{t \in[0,1 / 2]}|H(v(t))| \geq \delta .
$$


Combining (44) and (45) the assertion of Step 2 follows.

Step 3. We prove the lemma.

Let $\epsilon_{1}$ and $\delta$ be as in Step 2. We set

$$
\gamma:=\min \left\{\epsilon_{1}, \frac{\delta}{2}\right\} \text {. }
$$

If $\left\|\partial_{t} v-\eta \chi X_{H}(v)-\dot{\rho} X_{F_{\rho}}(v)\right\|_{2} \geq \gamma$ the assertion of the lemma follows by looking at the first component of the gradient in (39). If $\left\|\partial_{t} v-\eta \chi X_{H}(v)-\dot{\rho} X_{F_{\rho}}(v)\right\|_{2}<\gamma$ then the assertion of the lemma follows from Step 2 by looking at the second component of the gradient and noting that $\chi$ has support in $(0,1 / 2)$ and integrates to one. This completes the proof of Lemma 3.9.

Proof of Theorem 1.2 continued. To simplify notation we will drop in the following the superscripts $\chi$ and $\rho$ and write $H$ for $H^{\chi}$ and $F$ for $F^{\rho}$. To prove that $H F\left(\mathscr{A}^{H}\right)=0$ we look at two $s$-dependent action functionals $\mathscr{A}_{F_{s}}^{H}$ and $\mathscr{A}_{\bar{F}}^{H}$ which interpolate between $\mathscr{A}^{H}$ and $\mathscr{A}_{F}^{H}$, respectively between $\mathscr{A}_{F}^{H}$ and $\mathscr{A}^{H}$. Since the norm of the gradient of $\mathscr{A}_{F}^{H}$ is positively bounded from below, we will see that there are no finite energy gradient flow lines for the $s$-dependent functionals $\mathscr{A}_{F_{s}}^{H}$ and $\mathscr{A}_{\bar{F}}^{H}$. To prove $H F\left(\mathscr{A}^{H}\right)=0$ we glue $F_{s}$ and $\bar{F}_{s}$ together and homotope it to zero. For these homotopies of homotopies we prove compactness modulo breaking for the moduli spaces of gradient flow lines.

Choose a smooth monotone cutoff function $\beta \in C^{\infty}(\mathbb{R},[0,1])$ satisfying $\beta(s)=$ 1 for $s \geq 1$ and $\beta(s)=0$ for $s \leq 0$. Define

$$
F_{s}=\beta(s) F, \quad \bar{F}_{s}=(1-\beta(s)) F .
$$

For $R \geq 1$ we define a parametrized family of concatenations of $F_{S}$ and $\bar{F}_{S}$ by

$$
\bar{F}_{s} \#_{R} F_{S}= \begin{cases}F_{S+R} & s \leq 0 \\ \bar{F}_{s-R} & s \geq 0\end{cases}
$$

We prove the theorem now in three steps.

Step 1. There exists a constant $R_{0}$ depending only on $H$ and the action values of $\mathscr{A}^{H}$ at $\left(v^{ \pm}, \eta^{ \pm}\right)$with the property that if $R \geq R_{0}$, then there are no flow lines of the s-dependent gradient $\nabla A_{\bar{F}_{s} \#_{R}}^{H} F_{s}$ converging asymptotically to $\left(v^{ \pm}, \eta^{ \pm}\right)$.

Let $\gamma>0$ be as in Lemma 3.9. We abbreviate

$$
\|F\|_{\infty}=\max _{t \in[0,1], x \in V}\left|F_{t}(x)\right| .
$$

Choose $R_{0} \in \mathbb{R}$ such that

$$
R_{0}>\frac{\mathscr{A}^{H}\left(v^{+}, \eta^{+}\right)-\mathscr{A}^{H}\left(v^{-}, \eta^{-}\right)+2\|F\|_{\infty}}{2 \gamma^{2}}+\frac{1}{2} .
$$


Let $R \geq R_{0}$. We assume by contradiction that $w(s)=(v, \eta)(s)$ is a flow line of $\nabla A_{\bar{F}_{s} \#_{R} F_{s}}$ converging asymptotically to $\left(v^{ \pm}, \eta^{ \pm}\right)$. We estimate using Lemma 3.9

$$
\begin{aligned}
0 & \leq \int_{-\infty}^{-R+1}\left\|\partial_{s} w\right\|^{2} d s+\int_{R}^{\infty}\left\|\partial_{s} w\right\|^{2} d s \\
& =\int_{-\infty}^{\infty}\left\|\partial_{s} w\right\|^{2} d s-\int_{-R+1}^{R}\left\|\partial_{s} w\right\|^{2} d s \\
& =\mathscr{A}^{H}\left(v^{+}, \eta^{+}\right)-\mathscr{A}^{H}\left(v^{-}, \eta^{-}\right)-\int_{-\infty}^{\infty}\left(\partial_{s} \mathscr{A}_{\bar{F}_{s} \#_{R} F_{s}}\right)(w) d s \\
& \quad-\int_{-R+1}^{R}\left\|\nabla \mathscr{A}_{F}^{H}(w)\right\|^{2} d s \\
& \leq \mathscr{A}^{H}\left(v^{+}, \eta^{+}\right)-\mathscr{A}^{H}\left(v^{-}, \eta^{-}\right)+2\|F\|_{\infty}-(2 R-1) \gamma^{2}<0 .
\end{aligned}
$$

This contradiction shows that $w$ cannot exist and hence Step 1 follows.

Step 2. Let $F: V \rightarrow \mathbb{R}$ be a Hamiltonian (not necessarily displacing $\Sigma$ ) such that $X_{F}$ has compact support. Assume that $R \geq 1$ and $w(s)=(v, \eta)(s)$ is a flow line of $\nabla A_{\bar{F}_{s} \#_{R} F_{s}}$ which converges asymptotically to $\left(v^{ \pm}, \eta^{ \pm}\right)$. Then the Lagrange multiplier $\eta(s)$ is bounded uniformly by a constant depending only on $H,\|F\|_{\infty}$, $R$ and the action values of $\mathscr{A}^{H}$ at $\left(v^{ \pm}, \eta^{ \pm}\right)$.

Let $\epsilon>0$ be the constant in Proposition 3.2. For $\sigma \in \mathbb{R}$ define $\tau(\sigma) \geq 0$ by

$$
\tau(\sigma):=\inf \left\{\tau \geq 0:\left\|\nabla A_{\bar{F}_{s} \#_{R} F_{s}}^{H}(w)(\sigma+\tau)\right\|<\epsilon, \sigma+\tau \notin(-R, R+1)\right\} .
$$

According to (33) the energy $E$ of the gradient flow line $w$ is bounded from above uniformly by

$$
E \leq \mathscr{A}^{H}\left(v^{+}, \eta^{+}\right)-\mathscr{A}^{H}\left(v^{-}, \eta^{-}\right)+2\|F\|_{\infty} .
$$

From this we obtain as in (12) the estimate

$$
\tau(\sigma) \leq \frac{\mathscr{A}^{H}\left(v^{+}, \eta^{+}\right)-\mathscr{A}^{H}\left(v^{-}, \eta^{-}\right)+2\|F\|_{\infty}}{\epsilon^{2}}+2 R+1=: \kappa_{1} .
$$

Since $\sigma+\tau(\sigma) \notin(-R, R+1)$ we have

$$
\nabla \mathscr{A}_{\bar{F}_{s} \#_{R} F_{s}}^{H}(w)(\sigma+\tau(\sigma))=\nabla \mathscr{A}^{H}(w)(\sigma+\tau(\sigma)) .
$$

Observe further that for all $s \in \mathbb{R}$ the absolute action value at $w(s)$ is uniformly bounded by

$$
\left|\mathscr{A}_{\bar{F}_{s} \#_{R} F_{s}}^{H}(w(s))\right| \leq \max \left\{\left|\mathscr{A}^{H}\left(v^{+}, \eta^{+}\right)\right|,\left|\mathscr{A}^{H}\left(v^{-}, \eta^{-}\right)\right|\right\}+2\|F\|_{\infty}=: \kappa_{2} .
$$

Let $c$ be the constant appearing in Proposition 3.2. Combining Proposition 3.2 with (47) and (48) we get the estimate

$$
|\eta(\sigma+\tau(\sigma))| \leq c\left(\kappa_{2}+1\right) .
$$


The second component in the gradient flow Equation (30) for $\mathscr{A}_{\bar{F}_{s}} \#_{R} F_{S}$ shows that

$$
\left|\partial_{s} \eta\right| \leq\|H\|_{\infty}=\max _{x \in V}|H(x)| .
$$

Combining (46), (49), and (50) we obtain

$$
|\eta(\sigma)| \leq|\eta(\sigma+\tau(\sigma))|+\int_{\sigma}^{\sigma+\tau(\sigma)}\left|\partial_{s} \eta\right| d s \leq c\left(\kappa_{2}+1\right)+\|H\|_{\infty} \kappa_{1} .
$$

Since $\kappa_{1}$ and $\kappa_{2}$ depend only on $H,\|F\|_{\infty}, R$ and the asymptotic action values Step 2 follows.

Step 3. We prove the theorem.

Let $F$ be a displacing Hamiltonian and $R_{0}$ the constant from Step 1 . Set $R:=R_{0}$ and consider the Hamiltonians $r F$ for $0 \leq r \leq 1$. Step 2 provides a uniform bound on the Lagrange multiplier along gradient flow lines of $\mathscr{A} \frac{H}{r F_{s} \#_{R} r F_{s}}$ for all $r \in[0,1]$, which implies compactness modulo breaking for this homotopy in $r$. The usual argument in Floer theory shows that the maps on Floer homology

$$
\psi_{r}: H F\left(\mathscr{A}^{H}\right) \rightarrow H F\left(\mathscr{A}^{H}\right)
$$

induced by counting gradient flow lines of $\mathscr{A} \frac{H}{r F_{s} \#_{R}} r F_{s}$ are independent of $r$. Since $\psi_{1}=0$ by Step 1 and since $\psi_{0}=\mathrm{id}$, this proves that $H F\left(\mathscr{A}^{H}\right)=0$, and hence completes the proof of Theorem 1.2.

\section{Index computations}

In this section we prove Theorem 1.3. The proof comes down to the computation of the indices of generators of the Floer chain complex in the case that $\Sigma$ is the unit cotangent bundle of the sphere.

We first have to study the question under which conditions $\operatorname{RFH}(\Sigma, V)$ has a $\mathbb{Z}$-grading. Throughout this section, we make the following assumptions:

(A) Closed Reeb orbits on $(\Sigma, \alpha)$ are of Morse-Bott type (see Section 3).

(B) $\Sigma$ is simply connected and $V$ satisfies $I_{c_{1}}=0$.

Under these assumptions the (transversal) Conley Zehnder index of a Reeb orbit $v \in C^{\infty}\left(S^{1}, \Sigma\right)$ can be defined in the following way. Since $\Sigma$ is simply connected, we can find a map $\bar{v} \in C^{\infty}(D, \Sigma)$ on the unit disk $D=\{z \in \mathbb{C}:|z| \leq 1\}$ such that $\bar{v}\left(e^{2 \pi i t}\right)=v(t)$. Choose a (homotopically unique) symplectic trivialization of the symplectic vector bundle $\left(\bar{v}^{*} \xi, \bar{v}^{*} d \alpha\right)$. The linearized flow of the Reeb vector field along $v$ defines a path in the group $\operatorname{Sp}(2 n-2, \mathbb{R})$ of symplectic matrices. The Maslov index of this path [Robbin and Salamon 1993a] is the (transversal) Conley-Zehnder index $\mu_{C Z} \in \frac{1}{2} \mathbb{Z}$. It is independent of the choice of the disk $\bar{v}$ due to the assumption $I_{c_{1}}=0$ on $V$. 
Let $M$ be the moduli space of all finite energy gradient flow lines of the action functional $\mathscr{A}^{H}$. Since $\mathscr{A}^{H}$ is Morse-Bott every finite energy gradient flow line $(v, \eta) \in C^{\infty}\left(\mathbb{R} \times S^{1}, V\right) \times C^{\infty}(\mathbb{R}, \mathbb{R})$ converges exponentially at both ends to critical points $\left(v^{ \pm}, \eta^{ \pm}\right) \in \operatorname{crit}\left(\mathscr{A}^{H}\right)$ as the flow parameter goes to $\pm \infty$. The linearization of the gradient flow equation along any path $(v, \eta)$ in $\mathscr{L} \times \mathbb{R}$ which converges exponentially to the critical points of $\mathscr{A}^{H}$ gives rise to an operator $D_{(v, \eta)}^{\mathscr{A}^{H}}$. For suitable weighted Sobolev spaces (the weights are needed because we are in a Morse-Bott situation) the operator $D_{(v, \eta)}^{A^{H}}$ is a Fredholm operator. Let $C^{-}, C^{+} \subset$ $\operatorname{crit}\left(\mathscr{A}^{H}\right)$ be the connected components of the critical manifold of $\mathscr{A}^{H}$ containing $\left(v^{-}, \eta^{-}\right)$or $\left(v^{+}, \eta^{+}\right)$respectively. The local virtual dimension of $\mathcal{M}$ at a finite energy gradient flow line is defined to be

$$
\operatorname{virdim}_{(v, \eta)} M:=\operatorname{ind} D_{(v, \eta)}^{\otimes^{H}}+\operatorname{dim} C^{-}+\operatorname{dim} C^{+}
$$

where $\operatorname{ind} D_{(v, \eta)}^{\star^{H}}$ is the Fredholm index of the Fredholm operator $D_{(v, \eta)}^{\otimes^{H}}$. For generic compatible almost complex structures, the moduli space of finite energy gradient flow lines is a manifold and the local virtual dimension of the moduli space at a gradient flow line $(v, \eta)$ corresponds to the dimension of the connected component of $\mathcal{M}$ containing $(v, \eta)$. Our first goal is to prove the following index formula.

Proposition 4.1. Assume hypotheses $(A)$ and $(B)$ hold. Let $C^{-}, C^{+} \subset \operatorname{crit}\left(A^{H}\right)$ be two connected components of the critical manifold of $\mathscr{A}^{H}$. Let $(v, \eta) \in C^{\infty}(\mathbb{R} \times$ $\left.S^{1}, V\right) \times C^{\infty}(\mathbb{R}, \mathbb{R})$ be a gradient flow line of $\mathscr{A}^{H}$ which converges at both ends $\lim _{s \rightarrow \pm \infty}(v, \eta)(s) \rightarrow\left(v^{ \pm}, \eta^{ \pm}\right)$to critical points of $\mathscr{A}^{H}$ satisfying $\left(v^{ \pm}, \eta^{ \pm}\right) \in C^{ \pm}$. Choose maps $\bar{v}^{ \pm} \in C^{\infty}(D, \Sigma)$ satisfying $\bar{v}^{ \pm}\left(e^{2 \pi i t}\right)=v^{ \pm}(t)$. Then the local virtual dimension of the moduli space $\mathcal{M}$ of finite energy gradient flow lines of $\mathscr{A}^{H}$ at $(v, \eta)$ is given by

$$
\begin{array}{r}
\operatorname{virdim}_{(v, \eta)} \mathcal{M}=\mu_{C Z}\left(v^{+}\right)-\mu_{C Z}\left(v^{-}\right) \\
+2 c_{1}\left(\bar{v}^{-} \# v \# \bar{v}^{+}\right) \\
+\frac{\operatorname{dim} C^{-}+\operatorname{dim} C^{+}}{2}
\end{array}
$$

where $\bar{v}^{-} \# v \# \bar{v}^{+}$is the sphere obtained by capping the cylinder $v$ with the disks $\bar{v}^{+}$and $\bar{v}^{-}$, and $c_{1}=c_{1}(T V)$.

The proof is based on a discussion of spectral flows.

Spectral flows. It is shown in [Robbin and Salamon 1995] that the Fredholm index of $D_{(v, \eta)}^{\nexists^{H}}$ can be computed via the spectral flow $\mu_{\text {spec }}$ (see Appendix C) of the

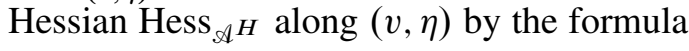

$$
\operatorname{ind} D_{(v, \eta)}^{\unlhd^{H}}=\mu_{\text {spec }}\left(\operatorname{Hess}_{\mathscr{A}^{H}}(v, \eta)\right) .
$$


Our proof compares the spectral flow of the Hessian of $\mathscr{A}^{H}$ with the spectral flow of the action functional of classical mechanics which can be computed via the Conley-Zehnder indices. For a fixed Lagrange multiplier $\eta \in \mathbb{R}$ the action functional of classical mechanics arises as

$$
\mathscr{A}_{\eta}^{H}:=\mathscr{A}^{H}(\cdot, \eta): \mathscr{L} \rightarrow \mathbb{R} .
$$

Assume first that the periods $\eta^{ \pm}$of the Reeb orbits $v^{ \pm}$are nonzero. We begin by homotoping the action functional $\mathscr{A}^{H}$ via Morse-Bott functionals with fixed critical manifold to an action functional $\mathscr{A}^{H^{1}}$ which satisfies the assumptions of (the infinite dimensional analogue of) Lemma C.6. There exists a neighbourhood $U \subset V$ of $\Sigma$ and $\epsilon>0$ such that $U$ is symplectomorphic to $(\Sigma \times(1-\epsilon, 1+\epsilon), d(r \alpha))$, where $r$ is the coordinate on $(1-\epsilon, 1+\epsilon)$. Since $\mathscr{A}^{H}$ is Morse-Bott and the Hamiltonian vector field $X_{H}(x)$ for $x \in \Sigma$ equals the Reeb vector field $R(x)$, there exists a homotopy $H^{s}$ for $s \in[0,1]$ which satisfies the following conditions:

- $H^{0}=H$.

- $X_{H^{s}}(x)=R(x)$ for $x \in \Sigma$ and $s \in[0,1]$.

- There exist neighbourhoods $U^{ \pm} \subset U$ of the critical manifolds $C^{ \pm}$and functions $h_{ \pm} \in C^{\infty}((1-\epsilon, 1+\epsilon))$ satisfying $h_{ \pm}(1)=0, h_{ \pm}^{\prime}(1)=1, h_{ \pm}^{\prime \prime}(1) \neq 0$, and $h_{ \pm}^{\prime}(r) \neq 0$ for $r \in(1-\epsilon, 1+\epsilon)$ such that $H^{1}(x, r)=h_{ \pm}(r)$ for $(x, r) \in$ $U^{ \pm} \subset \Sigma \times(1-\epsilon, 1+\epsilon)$.

- $\mathscr{A}^{H^{s}}$ is Morse-Bott for all $s \in[0,1]$.

Here the signs of $h_{ \pm}^{\prime \prime}(0)$ are determined by the second derivatives of $H$ in the direction transverse to $\Sigma$ along $C^{ \pm}$. Since $\mathscr{A}^{H}$ can be homotoped to $\mathscr{A}^{H^{1}}$ via Morse-Bott action functionals with fixed critical manifold, we obtain

$$
\mu_{\text {spec }}\left(\operatorname{Hess}_{\mathscr{A}^{H}}(v, \eta)\right)=\mu_{\text {spec }}\left(\operatorname{Hess}_{\mathscr{A l}^{1} H^{1}}(v, \eta)\right) .
$$

If $\left(v_{0}, \eta_{0}\right) \in C^{\infty}\left(S^{1}, \Sigma \cap U^{ \pm}\right) \times \mathbb{R}$ is a critical point of $\mathscr{A}^{H}$, then $\left(v_{0}, \eta_{0}\right)$ is also a critical point of $\mathscr{A}^{H^{1}}$. Moreover, the family $\left(v_{\rho}, \eta_{\rho}\right) \in C^{\infty}\left(S^{1}, U\right) \times \mathbb{R}$ given by

$$
v_{\rho}(t)=\left(v_{0}(t), h^{-1}(-\rho)\right), \quad \eta_{\rho}=\frac{\eta_{0}}{h^{\prime}\left(h^{-1}(-\rho)\right)}
$$

consists of critical points for the family of action functionals $\mathscr{A}^{H^{1}, \rho}: \mathscr{L} \times \mathbb{R} \rightarrow \mathbb{R}$ given for $(v, \eta) \in \mathscr{L} \times \mathbb{R}$ by

$$
\mathscr{A}^{H^{1}, \rho}(v, \eta):=\int v^{*} \lambda-\eta\left(\int_{0}^{1} H^{1}(v(t)) d t+\rho\right) .
$$

Note that

$$
\left.\partial_{\rho} \eta_{\rho}\right|_{\rho=0}=-\frac{\eta_{0} h_{ \pm}^{\prime \prime}(1)}{h_{ \pm}^{\prime}(1)^{2}} .
$$


Recalling that $h_{ \pm}^{\prime}(1)=1$ we see that for $\eta_{0}=\eta^{ \pm} \neq 0$ the hypotheses of Lemma C.6 are satisfied. It follows from Theorem C.5 and Lemma C.6 that the spectral flow can be expressed in terms of the spectral flow of the action functional of classical mechanics plus a correction term accounting for the second derivatives of $H$ transversally to $\Sigma$ as

$$
\begin{aligned}
& \mu_{\text {spec }}\left(\operatorname{Hess}_{\mathscr{A l}^{H^{1}}}(v, \eta)\right) \\
& \quad=\mu_{\mathrm{spec}}\left(\operatorname{Hess}_{\mathscr{A}_{\eta}^{H^{1}}}(v)\right)+\frac{1}{2}\left(\operatorname{sign}\left(\eta^{-} \cdot h_{-}^{\prime \prime}(0)\right)-\operatorname{sign}\left(\eta^{+} \cdot h_{+}^{\prime \prime}(0)\right)\right) .
\end{aligned}
$$

It follows from a theorem due to Salamon and Zehnder [1992] that the spectral flow of the Hessian of $\mathscr{A}_{\eta}^{H^{1}}$ can be computed via Conley-Zehnder indices. However, the Conley-Zehnder indices in the Salamon-Zehnder theorem are not the (transversal) Conley-Zehnder indices explained above, but the Maslov index of the linearized flow of the Reeb vector field on the whole tangent space of $V$ and not just on the contact hyperplane. For a Reeb orbit $v$ we will denote this second (full) ConleyZehnder index by $\hat{\mu}_{C Z}(v)$. Note that $\hat{\mu}_{C Z}(v)$ depends on the second derivatives of $H$ transversally to $\Sigma$ while $\mu_{C Z}(v)$ does not. Another complication is that we are in a Morse-Bott situation and we have to adapt the Salamon-Zehnder theorem to this situation. Formula (74) defines the spectral flow also for Morse-Bott situations. To adapt the Conley-Zehnder indices to the Morse-Bott situation observe that in a symplectic trivialization the linearized flow of the Reeb vector field can be expressed as a solution of an ordinary differential equation

$$
\dot{\Psi}(t)=J_{0} S(t) \Psi(t), \quad \Psi(0)=\mathrm{id},
$$

where $t \mapsto S(t)=S(t)^{T}$ is a smooth path of symmetric matrices. For a real number $\delta$ we define $\Psi_{\delta}$ as the solution of

$$
\dot{\Psi}_{\delta}(t)=J_{0}(S(t)-\delta \cdot \mathrm{id}) \Psi_{\delta}(t), \quad \Psi_{\delta}(0)=\mathrm{id},
$$

and set $\mu_{C Z}^{\delta}(v)$, respectively $\hat{\mu}_{C Z}^{\delta}(v)$ as the Conley-Zehnder index of $\Psi_{\delta}$ where in the first case we restrict $\Psi_{\delta}$ to the contact hyperplane and in the second case we consider it on the whole tangent space. We put

$$
\mu_{C Z}^{+}(v):=\lim _{\delta \searrow 0} \mu_{C Z}^{\delta}(v), \quad \mu_{C Z}^{-}(v):=\lim _{\delta \searrow 0} \mu_{C Z}^{-\delta}(v)
$$

and analogously $\hat{\mu}_{C Z}^{+}(v)$ and $\hat{\mu}_{C Z}^{-}(v)$. Note that while $\hat{\mu}_{C Z}(v)$ and $\mu_{C Z}(v)$ are half-integers, $\hat{\mu}_{C Z}^{ \pm}(v)$ and $\mu_{C Z}^{ \pm}(v)$ are actually integers. We are now in position to state the theorem of Salamon and Zehnder. 
Theorem 4.2 (Salamon-Zehnder [Salamon and Zehnder 1992]). The spectral flow of the Hessian of $\mathscr{A}_{\eta}^{H^{1}}$ is given by

$$
\mu\left(H_{\mathscr{A}_{\eta} H^{1}}(v)\right)=\hat{\mu}_{C Z}^{+}\left(v^{+}\right)-\hat{\mu}_{C Z}^{-}\left(v^{-}\right)+2 c_{1}\left(\bar{v}^{-} \# v \# \bar{v}^{+}\right) .
$$

Relations between Conley-Zehnder indices. The following two lemmata relate the different Conley-Zehnder indices to each other.

Lemma 4.3. For a Reeb orbit $v$ with period $\eta \neq 0$, viewed as a 1-periodic orbit of the Hamiltonian vector field of $\eta H$, we have

$$
\hat{\mu}_{C Z}^{ \pm}(v)=\mu_{C}^{ \pm}(v)+\frac{1}{2}\left(\operatorname{sign}\left(\eta h^{\prime \prime}(0)\right) \mp 1\right) .
$$

Proof. By the product property [Salamon 1999] of the Conley-Zehnder index the difference of $\hat{\mu}_{C Z}^{ \pm}(v)$ and $\mu_{C Z}^{ \pm}(v)$ is given by the Conley-Zehnder index of the linearized flow of the Hamiltonian vector field restricted to the symplectic orthogonal complement $\xi^{\omega}$ of the contact hyperplane in the tangent space of $V$. With respect to the trivialization $\mathbb{C} \rightarrow \xi^{\omega}(v(t))$ given by $x+i y \mapsto\left(x \cdot \nabla H(v(t))+y \cdot X_{H}(v(t))\right)$ for $t \in S^{1}$, the linearized flow $\Psi(t)$ of the Hamiltonian vector field satisfies the differential equation

$$
\dot{\Psi}(t)=\left(\begin{array}{cc}
0 & 0 \\
\eta h^{\prime \prime}(0) & 0
\end{array}\right) \Psi(t), \quad \Psi(0)=\mathrm{id}
$$

and is thus given by

$$
\Psi(t)=\left(\begin{array}{cc}
1 & 0 \\
t \eta h^{\prime \prime}(0) & 1
\end{array}\right)
$$

Abbreviate $a:=\eta \cdot h^{\prime \prime}(0)$. Then the perturbed flow $\Psi_{\delta}(t)$ satisfies the differential equation

$$
\dot{\Psi}_{\delta}(t)=\left(\begin{array}{cc}
0 & \delta \\
a-\delta & 0
\end{array}\right) \Psi_{\delta}(t), \quad \Psi_{\delta}(0)=\mathrm{id} .
$$

The matrix in this equation has eigenvalues $\pm \lambda$, where

$$
\lambda:= \begin{cases}\sqrt{\delta(a-\delta)} & \delta(a-\delta)>0, \\ i \sqrt{-\delta(a-\delta)} & \delta(a-\delta)<0 .\end{cases}
$$

Diagonalizing the matrix, we find the solution

$$
\begin{aligned}
\Psi_{\delta}(t) & =\frac{1}{2 \delta \lambda}\left(\begin{array}{cc}
\delta & \delta \\
-\lambda & \lambda
\end{array}\right)\left(\begin{array}{cc}
e^{-\lambda t} & 0 \\
0 & e^{\lambda t}
\end{array}\right)\left(\begin{array}{cc}
\lambda & -\delta \\
\lambda & \delta
\end{array}\right) \\
& =\frac{1}{2 \delta \lambda}\left(\begin{array}{cc}
\delta \lambda\left(e^{\lambda t}+e^{-\lambda t}\right) & \delta^{2}\left(e^{\lambda t}-e^{-\lambda t}\right) \\
\lambda^{2}\left(e^{\lambda t}-e^{-\lambda t}\right) & \delta \lambda\left(e^{\lambda t}+e^{-\lambda t}\right)
\end{array}\right) .
\end{aligned}
$$


Inserting $\lambda^{2}=\delta(a-\delta)$ and expanding in powers of $\delta$ yields

$$
\Psi_{\delta}(t)=(1+O(\delta))\left(\begin{array}{cc}
1 & \delta t \\
(a-\delta) t & 1
\end{array}\right) .
$$

Recall from [Salamon 1999] that the Conley-Zehnder index can be computed in terms of crossing numbers, where a number $t \in[0,1]$ is called a crossing if the equality $\operatorname{det}\left(\mathrm{id}-\Psi_{\delta}(t)\right)=0$ holds. The formula above shows that for $\delta$ small enough the only crossing happens at zero. Hence by [Salamon 1999] the ConleyZehnder index is given by

$$
\mu_{C Z}\left(\Psi_{\delta}\right)=\frac{1}{2} \operatorname{sign}\left(\begin{array}{cc}
a-\delta & 0 \\
0 & -\delta
\end{array}\right) .
$$

If $|\delta|<|a|$ we obtain

$$
\mu_{C Z}\left(\Psi_{\delta}\right)=\frac{1}{2}(\operatorname{sign}(a)-\operatorname{sign}(\delta))=\frac{1}{2}\left(\operatorname{sign}\left(\eta h^{\prime \prime}(0)\right)-\operatorname{sign}(\delta)\right)
$$

and hence

$$
\hat{\mu}_{C Z}^{ \pm}(v)-\mu_{C Z}^{ \pm}(v)=\frac{1}{2}\left(\operatorname{sign}\left(\eta h^{\prime \prime}(0)\right) \mp 1\right) .
$$

Lemma 4.4. Let $v$ be a Reeb orbit with period $\eta \neq 0$ and $C_{v}$ the component of the critical manifold of $\mathscr{A}^{H^{1}}$ which contains $v$. Then

$$
\hat{\mu}_{C Z}(v)=\hat{\mu}_{C Z}^{ \pm}(v) \pm \frac{\operatorname{dim} C_{v}}{2}, \quad \mu_{C Z}(v)=\mu_{C Z}^{ \pm}(v) \pm \frac{\operatorname{dim} C_{v}-1}{2} .
$$

Proof. Obviously

$$
\hat{\mu}_{C Z}^{-}(v)-\hat{\mu}_{C Z}^{+}(v)=\operatorname{dim} C_{v}, \quad \mu_{C Z}^{-}(v)-\mu_{C Z}^{+}(v)=\operatorname{dim} C_{v}-1 .
$$

The reason for the minus one in the second formula is that the transversal ConleyZehnder index only takes into account the critical manifold of $\mathscr{A}^{H^{1}}$ modulo the $S^{1}$ action given by the Reeb vector field. The Conley-Zehnder index can be interpreted as intersection number of a path of Lagrangian subspaces with the Maslov cycle; see [Robbin and Salamon 1993a]. Under a small perturbation the intersection number can only change at the initial and endpoint. Since the Lagrangian subspace at the initial point is fixed it will change only at the endpoint. There the contribution is given by half of the crossing number which equals $\operatorname{dim} C_{v}$ in the case one considers the Conley-Zehnder index on the whole tangent space respectively $\operatorname{dim} C_{v}-1$ if one considers the Conley-Zehnder index only on the contact hyperplane. In particular,

$$
\left|\hat{\mu}_{C Z}(v)-\hat{\mu}_{C Z}^{ \pm}(v)\right| \leq \frac{\operatorname{dim} C_{v}}{2}, \quad\left|\mu_{C Z}(v)-\mu_{C Z}^{ \pm}(v)\right| \leq \frac{\operatorname{dim} C_{v}-1}{2} .
$$

Comparing (56) and (57) the lemma follows. 
Proof of Proposition 4.1. We first assume that $\eta^{-}$and $\eta^{+}$are nonzero. Combining the theorem of Salamon and Zehnder (Theorem 4.2) with Lemma 4.3 and Lemma 4.4 we obtain

$$
\begin{aligned}
\mu\left(H_{\mathscr{A}_{\eta}^{H^{1}}}(v)\right)=\hat{\mu}_{C Z}^{+}\left(v^{+}\right) & -\hat{\mu}_{C Z}^{-}\left(v^{-}\right)+2 c_{1}\left(\bar{v}^{-} \# v \# \bar{v}^{+}\right) \\
=\mu_{C Z}^{+}\left(v^{+}\right) & -\mu_{C Z}^{-}\left(v^{-}\right)+2 c_{1}\left(\bar{v}^{-} \# v \# \bar{v}^{+}\right)-1 \\
& +\frac{1}{2}\left(\operatorname{sign}\left(\eta^{+} \cdot h_{+}^{\prime \prime}(0)\right)-\operatorname{sign}\left(\eta^{-} \cdot h_{-}^{\prime \prime}(0)\right)\right) \\
=\mu_{C Z}\left(v^{+}\right) & -\mu_{C Z}\left(v^{-}\right)+2 c_{1}\left(\bar{v}^{-} \# v \# \bar{v}^{+}\right)-\frac{\operatorname{dim} C^{-}+\operatorname{dim} C^{+}}{2} \\
& +\frac{1}{2}\left(\operatorname{sign}\left(\eta^{+} \cdot h_{+}^{\prime \prime}(0)\right)-\operatorname{sign}\left(\eta^{-} \cdot h_{-}^{\prime \prime}(0)\right)\right) .
\end{aligned}
$$

Combining this equality with (51), (53), (54), and (55) we compute

$$
\begin{aligned}
\operatorname{virdim}_{(v, \eta)} \mu & =\operatorname{ind} D_{(v, \eta)}^{\rtimes^{H}}+\operatorname{dim} C^{-}+\operatorname{dim} C^{+} \\
& =\mu\left(H_{A_{A} H}(v, \eta)\right)+\operatorname{dim} C^{-}+\operatorname{dim} C^{+} \\
& =\mu\left(H_{A^{H} H^{1}}(v, \eta)\right)+\operatorname{dim} C^{-}+\operatorname{dim} C^{+} \\
& =\mu\left(H_{A_{\eta}^{H^{1}}}(v)\right)+\frac{1}{2}\left(\operatorname{sign}\left(\eta^{-} \cdot h_{-}^{\prime \prime}(0)\right)-\operatorname{sign}\left(\eta^{+} \cdot h_{+}^{\prime \prime}(0)\right)\right) \\
& \quad+\operatorname{dim} C^{-}+\operatorname{dim} C^{+} \\
& =\mu_{C Z}\left(v^{+}\right)-\mu_{C Z}\left(v^{-}\right)+2 c_{1}\left(\bar{v}^{-} \# v \# \bar{v}^{+}\right) \\
& \quad+\frac{\operatorname{dim}\left(C^{-}\right)+\operatorname{dim}\left(C^{+}\right)}{2} .
\end{aligned}
$$

This proves the proposition for the case where the periods of the asymptotic Reeb orbits are both nonzero. To treat also the case where one of the asymptotic Reeb orbits is constant we consider the following involution on the loop space $\mathscr{L}$

$$
I(v)(t)=v(-t), \quad v \in \mathscr{L}, t \in S^{1} .
$$

We extend this involution to an involution on $\mathscr{L} \times \mathbb{R}$ which we denote by abuse of notation also by $I$ and which is given by

$$
I(v, \eta)=(I(v),-\eta), \quad(v, \eta) \in \mathscr{L} \times \mathbb{R} .
$$

The action functional $\mathscr{A}^{H}$ transforms under the involution $I$ by

$$
\mathscr{A}^{H}(I(v, \eta))=-\mathscr{A}^{H}(v, \eta), \quad(v, \eta) \in \mathscr{L} \times \mathbb{R} .
$$

In particular, the restriction of the involution $I$ to the critical manifold of $\mathscr{A}^{H}$ induces an involution on $\operatorname{crit}\left(\mathscr{A}^{H}\right)$ and the fixed points of this involution are the constant Reeb orbits. 
We consider now a finite energy gradient flow line $(v, \eta) \in C^{\infty}\left(\mathbb{R} \times S^{1}, V\right) \times$ $C^{\infty}(\mathbb{R}, \mathbb{R})$ of the action functional $\mathscr{A}^{H}$ whose right end $\left(v^{+}, \eta^{+}\right)$is a constant Reeb orbit and whose left end $\left(v^{-}, \eta^{-}\right)$is a nonconstant Reeb orbit. For the path $(v, \eta)$ in $\mathscr{L} \times \mathbb{R}$ we consider the path $(v, \eta)_{I}=\left(v_{I}, \eta_{I}\right)$ in $\mathscr{L} \times \mathbb{R}$ defined by $(v, \eta)_{I}(s)=$ $I(v, \eta)(-s)$ for $s \in \mathbb{R}$. The path $(v, \eta)_{I}$ goes from $\left(v^{+}, \eta^{+}\right)$to $I\left(v^{-}, \eta^{-}\right)$and gluing the paths $(v, \eta)$ and $(v, \eta)_{I}$ together we obtain a path $(v, \eta) \#(v, \eta)_{I}$ from $\left(v^{-}, \eta^{-}\right)$ to $I\left(v^{-}, \eta^{-}\right)$. The Fredholm indices of the different paths are related by

$$
\operatorname{ind} D_{(v, \eta)}^{\triangleleft^{H}}=\operatorname{ind} D_{(v, \eta)_{I}}^{\triangleleft^{H}}, \quad \operatorname{ind} D_{(v, \eta) \#(v, \eta)_{I}}^{\otimes^{H}}=\operatorname{ind} D_{(v, \eta)}^{\otimes^{H}}+\operatorname{ind} D_{(v, \eta)_{I}}^{\triangleleft^{H}}+\operatorname{dim} C^{+} .
$$

From this we compute, using (52) for the case of nonconstant Reeb orbits and the equality $\mu_{C Z}\left(I\left(v^{ \pm}\right)\right)=-\mu_{C Z}\left(v^{ \pm}\right)$,

$$
\begin{aligned}
\operatorname{ind} D_{(v, \eta)}^{\otimes^{H}}= & \frac{1}{2} \cdot \operatorname{ind} D_{(v, \eta) \#(v, \eta)_{I}}^{\triangleleft^{H}}-\frac{\operatorname{dim} C^{+}}{2} \\
= & \frac{1}{2}\left(\mu_{C Z}\left(I\left(v^{-}\right)\right)-\mu_{C Z}\left(v^{-}\right)+2 c_{1}\left(\bar{v}^{-} \# v \# v_{I} \# I \bar{v}^{+}\right)\right. \\
& \left.\quad-\frac{\operatorname{dim} C^{-}+\operatorname{dim} I C^{-}}{2}\right)-\frac{\operatorname{dim} C^{+}}{2} \\
= & -\mu_{C Z}\left(v^{-}\right)+2 c_{1}\left(\bar{v}^{-} \# v\right)-\frac{\operatorname{dim} C^{-}+\operatorname{dim} C^{+}}{2},
\end{aligned}
$$

from which we deduce (52) using (51). This proves the proposition for the case of gradient flow lines whose left end is a constant Reeb orbit. The case of gradient flow lines whose right end is constant can be deduced in the same way or by considering the coindex. This finishes the proof of the Proposition 4.1.

In order to define a $\mathbb{Z}$-grading on $\operatorname{RFH}(\Sigma, V)$ we need that the local virtual dimension just depends on the asymptotics of the finite energy gradient flow line. By (52) this is the case if $I_{c_{1}}=0$ on $V$. In this case the local virtual dimension is given by

$$
\operatorname{virdim}_{(v, \eta)} \mathcal{M}=\mu_{C Z}\left(v^{+}\right)-\mu_{C Z}\left(v^{-}\right)+\frac{\operatorname{dim} C^{-}+\operatorname{dim} C^{+}}{2} .
$$

In order to deal with the third term it is useful to introduce the following index for the Morse function $h$ on $\operatorname{crit}\left(\mathscr{A}^{H}\right)$. We define the signature index $\operatorname{ind}_{h}^{\sigma}(c)$ of a critical point $c$ of $h$ to be

$$
\operatorname{ind}_{h}^{\sigma}(c):=-\frac{1}{2} \operatorname{sign}\left(\operatorname{Hess}_{h}(c)\right),
$$

see Appendix A. The half signature index is related to the Morse index $\operatorname{ind}_{h}^{m}(c)$, given by the number of negative eigenvalues of $\operatorname{Hess}_{h}(c)$ counted with multiplicity, 
by

$$
\operatorname{ind}_{h}^{\sigma}(c)=\operatorname{ind}_{h}^{m}(c)-\frac{1}{2} \operatorname{dim}_{c}\left(\operatorname{crit}\left(\mathscr{A}^{H}\right)\right) .
$$

We define a grading $\mu$ on $C F_{*}\left(\mathscr{A}^{H}, h\right)$ by

$$
\mu(c):=\mu_{C Z}(c)+\operatorname{ind}_{h}^{\sigma}(c) .
$$

By considering the case of nondegenerate closed Reeb orbits, one sees that $\mu$ takes values in the set $\frac{1}{2}+\mathbb{Z}$, so it is indeed a $\mathbb{Z}$-grading (shifted by $\frac{1}{2}$ ). Using Equation (58), it is shown in Appendix A that the Floer boundary operator $\partial$ has degree -1 with respect to this grading. Hence we get a $\mathbb{Z}$-grading on the Rabinowitz Floer homology $R_{F}(\Sigma, V)$.

Proof of Theorem 1.3. We use the fact that the chain groups underlying the Floer homology $H F_{*}\left(\mathscr{A}^{H}\right)$ only depend on $(\Sigma, \alpha)$ and not on the embedding of $\Sigma$ into $V$. We show that for the unit cotangent bundle $S^{*} S^{n}$ for $n \geq 4$ the Floer homology equals the chain complex. More precisely, we choose the standard round metric on $S^{n}$ normalized such that all geodesics are closed with minimal period one. For this choice assumption (A) from Section 3.2 is satisfied. The critical manifold of $\mathscr{A}^{H}$ consists of $\mathbb{Z}$ copies of $S^{*} S^{n}$, where $\mathbb{Z}$ corresponds to the period of the geodesic. There is a Morse function $h_{0}$ on $S^{*} S^{n}$ with precisely 4 critical points and zero boundary operator (with $\mathbb{Z}_{2}$-coefficients!) whose Morse homology satisfies

$$
H M_{k}\left(S^{*} S^{n} ; \mathbb{Z}_{2}\right)=C M_{k}\left(h_{0} ; \mathbb{Z}_{2}\right)=\left\{\begin{array}{cl}
\mathbb{Z}_{2} & k \in\{0, n-1, n, 2 n-1\}, \\
0 & \text { else. }
\end{array}\right.
$$

Let $h$ be the Morse function on the critical manifold which coincides with $h_{0}$ on each connected component. The chain complex is generated by

$$
\operatorname{crit}(h) \cong \mathbb{Z} \times \operatorname{crit}\left(h_{0}\right) \text {. }
$$

A closed geodesic $c$ is also a critical point of the energy functional on the loop space. The index $\operatorname{ind}_{E}(c)$ of a closed geodesic is defined to be the Morse index of the energy functional at the geodesic and the nullity $v(c)$ is defined to be the dimension of the connected component of the critical manifold of the energy functional which contains the geodesic minus one. The (transverse) Conley-Zehnder index of a closed geodesic is given by

$$
\mu_{C Z}(c)=\operatorname{ind}_{E}(c)+\frac{v(c)}{2} .
$$

This is proved in [Duistermaat 1976; Weber 2002] for nondegenerate geodesics; the degenerate case follows from the nondegenerate one using the averaging property of the Conley-Zehnder index (Lemma 4.4). By the Morse index theorem (see [Morse 1996] or [Klingenberg 1995, Theorem 2.5.14]) the index of a geodesic is 
given by the number of conjugate points counted with multiplicity plus the concavity. The latter one vanishes for the standard round metric on $S^{n}$, since each closed geodesic has a variation of closed geodesics having the same length [Ziller 1977].

Using the Morse index theorem and equations (60) and (59), we compute the index of $(m, x) \in \mathbb{Z} \times \operatorname{crit}\left(h_{0}\right)$ :

$$
\begin{aligned}
\mu(m, x) & =\mu_{C Z}(m, x)+\operatorname{ind}_{h}^{\sigma}(x) \\
& =\operatorname{ind}_{E}(m, x)+\frac{v(m, x)}{2}+\operatorname{ind}_{h}^{\sigma}(x) \\
& =(2 m-1)(n-1)+\frac{2 n-2}{2}+\operatorname{ind}_{h}^{\sigma}(x) \\
& =2 m(n-1)+\operatorname{ind}_{h}^{m}(x)-\frac{2 n-1}{2} .
\end{aligned}
$$

It follows from Lemma 3.3 that the action satisfies

$$
\mathscr{A}^{H}(m, x)=m \text {. }
$$

In order to have a gradient flow line of $\mathscr{A}^{H}$ from a critical point $\left(m_{1}, x_{1}\right)$ to a critical point $\left(m_{2}, x_{2}\right)$ we need

$$
\begin{aligned}
\mathscr{A}^{H}\left(m_{2}, x_{2}\right)-\mathscr{A}^{H}\left(m_{1}, x_{1}\right) & =m_{2}-m_{1}>0, \\
\mu\left(m_{2}, x_{2}\right)-\mu\left(m_{1}, x_{1}\right) & =2\left(m_{2}-m_{1}\right)(n-1)+\left(i_{2}-i_{1}\right)=1
\end{aligned}
$$

for $i_{1}, i_{2} \in\{0, n-1, n, 2 n-1\}$, which is impossible if $n \geq 4$. Hence there are no gradient flow lines, so the Floer homology equals the chain complex. This proves Theorem 1.3.

\section{Appendix A: Morse-Bott homology}

We briefly indicate in this appendix how to define Morse-Bott homology by the use of gradient flow lines with cascades. More details of this approach can be found in [Frauenfelder 2004, Appendix A]. We begin with the finite dimensional situation. Let $M$ be a manifold and $f \in C^{\infty}(M)$ a Morse-Bott function, that is, the critical set $\operatorname{crit}(f)$ is a manifold and

$$
T_{x} \operatorname{crit}(f)=\operatorname{kerHess}_{f}(x), \quad x \in \operatorname{crit}(f),
$$

where $\operatorname{Hess}_{f}(x)$ denotes the Hessian of $f$ at $x$. We then choose an additional Morse function $h$ on $\operatorname{crit}(f)$. The chain group for Morse-Bott homology is the $\mathbb{Z}_{2}$-vector space given by

$$
\mathrm{CM}(f, h):=\operatorname{crit}(h) \otimes \mathbb{Z}_{2} .
$$




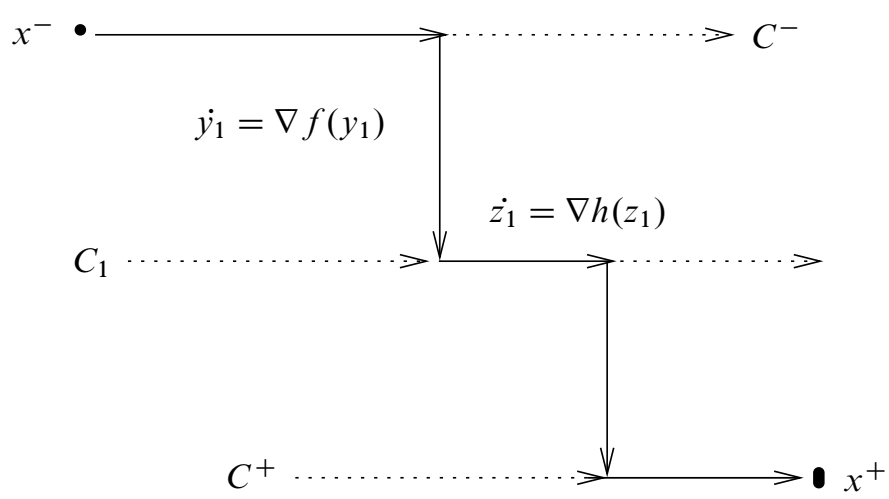

Figure 1. A flow line with cascades.

Morse-Bott homology should also be definable over the integers via the cascade approach, but this is nowhere written down. The boundary operator is defined by counting gradient flow lines with cascades between two critical points of $h$ which are indicated by Figure 1.

A gradient flow line with cascades starts with a gradient flow line of $h$ on $\operatorname{crit}(f)$ which converges at its negative asymptotic end to a critical point of $h$. In finite time this gradient flow line meets the asymptotic end of a gradient flow line of the Morse-Bott function $f$. We refer to this gradient flow line of $f$ as the first cascade. The cascade converges at its positive end again to a point in $\operatorname{crit}(f)$. There the flow continues with the gradient flow of $h$ on $\operatorname{crit}(f)$. After finite time a second cascade might appear but having passed through finitely many cascades we finally end up with a gradient flow line of $h$ which we follow until it converges asymptotically to a critical point of $h$. Gradient flow lines with zero cascades are also allowed. They correspond to ordinary Morse flow lines of the gradient of $h$ on the manifold $\operatorname{crit}(f)$. For a formal definition of gradient flow lines with cascades we refer to [Frauenfelder 2004].

We finally discuss the grading for Morse-Bott homology. If $f$ is Morse then the following two index conventions are often used. Either Morse homology is graded by the Morse index $\operatorname{ind}_{f}^{m}$, given by the dimensions of the negative eigenspaces, or by the signature index

$$
\operatorname{ind}_{f}^{\sigma}(x):=-\frac{1}{2} \operatorname{sign} \operatorname{Hess}_{f}(x), \quad x \in \operatorname{crit}(f),
$$

where sign denotes the signature of the quadratic form given by the difference of the dimensions of the positive and negative eigenspaces. The two indices are related by the following global shift

$$
\operatorname{ind}_{f}^{\sigma}=\operatorname{ind}_{f}^{m}-\frac{1}{2} \operatorname{dim}(M) .
$$


In particular, if $M$ is even dimensional then the signature index is integer valued and if $M$ is odd dimensional then it is half integer valued. The signature index plays an important role in Floer's semi-infinite dimensional Morse theory. There the stable and unstable manifolds are both infinite dimensional and hence the Morse index is infinite. The grading given is given by the Maslov index which can be interpreted as a signature index as explained in [Robbin and Salamon 1993b; 1996].

Both the Morse index and the signature index can be defined in the same way also for a Morse-Bott function $f$. The corresponding indices for a pair $(f, h)$ consisting of a Morse-Bott function $f$ and a Morse function $h$ on $\operatorname{crit}(f)$ are defined by taking sums $\operatorname{ind}_{f, h}^{m}(x):=\operatorname{ind}_{f}^{m}(x)+\operatorname{ind}_{h}^{m}(x), \quad \operatorname{ind}_{f, h}^{\sigma}(x):=\operatorname{ind}_{f}^{\sigma}(x)+\operatorname{ind}_{h}^{\sigma}(x), \quad x \in \operatorname{crit}(h)$. The shift formula (61) continues to hold for these indices in Morse-Bott theory.

Consider gradient flow lines with $k$ cascades between components $C_{0}, \ldots, C_{k}$ of crit $(f)$, starting at a critical point $x^{-}$of $h$ on $C^{-}=C_{0}$ and ending at a critical point $x^{+}$of $h$ on $C^{+}=C_{k}$. For generic metric, their moduli space (divided by the $\mathbb{R}$-actions on the cascades) $\mathcal{M}\left(x^{-}, x^{+} ; C_{0}, \ldots, C_{k}\right)$ is a manifold of dimension

$$
\begin{aligned}
\operatorname{dim} M\left(x^{-}, x^{+} ; C_{0}, \ldots, C_{k}\right) & \\
& =\operatorname{ind}_{h}^{m}\left(x^{+}\right)-\operatorname{ind}_{h}^{m}\left(x^{-}\right)-1+\sum_{i=1}^{k}\left(\operatorname{dim} M\left(C_{i-1}, C_{i}\right)-\operatorname{dim} C_{i}\right),
\end{aligned}
$$

where $M\left(C_{i-1}, C_{i}\right)$ is the moduli space of gradient flow lines of $f$ from $C_{i-1}$ to $C_{i}$ (not divided by the $\mathbb{R}$-action). From

$$
\operatorname{dim} M\left(C_{i-1}, C_{i}\right)=\operatorname{ind}_{f}^{m}\left(C_{i}\right)-\operatorname{ind}_{f}^{m}\left(C_{i-1}\right)+\operatorname{dim} C_{i}
$$

we obtain the dimension formula in terms of Morse indices:

$$
\begin{aligned}
\operatorname{dim} M\left(x^{-}, x^{+} ; C_{0},\right. & \left.\ldots, C_{k}\right) \\
& =\operatorname{ind}_{h}^{m}\left(x^{+}\right)-\operatorname{ind}_{h}^{m}\left(x^{-}\right)-1+\operatorname{ind}_{f}^{m}\left(C^{+}\right)-\operatorname{ind}_{f}^{m}\left(C^{-}\right) \\
& =\operatorname{ind}_{f, h}^{m}\left(x^{+}\right)-\operatorname{ind}_{f, h}^{m}\left(x^{-}\right)-1 .
\end{aligned}
$$

On the other hand, in the Morse-Bott case the Morse and signature indices of a critical component $C$ are related by

$$
\operatorname{ind}_{f}^{\sigma}(C)=\operatorname{ind}_{f}^{m}(C)-\frac{1}{2}(\operatorname{dim} M-\operatorname{dim} C) .
$$

Inserting this in Equation (62) yields

$$
\operatorname{dim} \mu\left(C_{i-1}, C_{i}\right)=\operatorname{ind}_{f}^{\sigma}\left(C_{i}\right)-\operatorname{ind}_{f}^{\sigma}\left(C_{i-1}\right)+\frac{\operatorname{dim} C_{i}+\operatorname{dim} C_{i-1}}{2},
$$


which in turn yields the dimension formula in terms of signature indices:

$$
\begin{aligned}
& \operatorname{dim} M\left(x^{-}, x^{+} ; C_{0}, \ldots, C_{k}\right) \\
& =\operatorname{ind}_{h}^{m}\left(x^{+}\right)-\operatorname{ind}_{h}^{m}\left(x^{-}\right)-1+\operatorname{ind}_{f}^{\sigma}\left(C^{+}\right)-\operatorname{ind}_{f}^{\sigma}\left(C^{-}\right)-\frac{\operatorname{dim} C^{+}}{2}+\frac{\operatorname{dim} C^{-}}{2} \\
& =\operatorname{ind}_{f, h}^{\sigma}\left(x^{+}\right)-\operatorname{ind}_{f, h}^{\sigma}\left(x^{-}\right)-1 .
\end{aligned}
$$

So we get the same formula for $\operatorname{dim} \mu\left(x^{-}, x^{+} ; C_{0}, \ldots, C_{k}\right)$ using either Morse indices or signature indices. Since this dimension equals zero for the moduli spaces contributing to the boundary operator in Morse-Bott homology, this shows that the boundary operator has degree -1 with respect to either grading. However, we would like to point out that a mixture of Morse indices and signature indices does not lead in general to a grading on Morse-Bott homology unless all the connected components of the critical manifold of $f$ have the same dimension.

Finally, consider the situation in Floer homology where the ambient space is infinite-dimensional, but the components of the critical set crit $(f)$ and the moduli spaces $M\left(C_{i-1}, C_{i}\right)$ are still finite-dimensional. Moreover, (under suitable hypotheses) the dimension of these moduli spaces can be expressed by a formula analogous to (64) in terms of Conley-Zehnder indices:

$$
\operatorname{dim} \mu\left(C_{i-1}, C_{i}\right)=\mu_{C Z}\left(C_{i}\right)-\mu_{C Z}\left(C_{i-1}\right)+\frac{\operatorname{dim} C_{i}+\operatorname{dim} C_{i-1}}{2} .
$$

See for example Equation (58) for the Floer homology considered in this paper. This suggests that the Conley-Zehnder index should be viewed as a signature index, and the same computation as in the finite dimensional case above yields the dimension formula

$$
\operatorname{dim} M\left(x^{-}, x^{+} ; C_{0}, \ldots, C_{k}\right)=\mu\left(x^{+}\right)-\mu\left(x^{-}\right)-1
$$

with respect to the signature index

$$
\mu(x):=\mu_{C Z}(x)+\operatorname{ind}_{h}^{\sigma}(x) .
$$

Thus the boundary operator in Floer homology has degree -1 with respect to $\mu$ and $\mu$ descends to an integer grading on Floer homology. Actually, in the case considered in this paper this grading takes values in $\frac{1}{2}+\mathbb{Z}$, where the shift by $\frac{1}{2}$ reflects the 1-dimensional constraint imposed on the free loop space.

\section{Appendix B: A Wasserman-type theorem for the Rabinowitz action functional}

Let $(V, \lambda)$ be an exact symplectic manifold of dimension $n \geq 2$ and let $\mathscr{L}=$ $C^{\infty}\left(S^{1}, V\right)$ be the free loop space. Denote by $C_{c}^{\infty}(V)$ the vector space of smooth 
functions on $V$ whose differential has compact support. Recall that for a Hamiltonian $H \in C_{c}^{\infty}(V)$ the Rabinowitz action functional

$$
\mathscr{A}^{H}: \mathscr{L} \times \mathbb{R} \rightarrow \mathbb{R}
$$

is given by

$$
\mathscr{A}^{H}(v, \eta)=\int_{0}^{1} v^{*} \lambda-\eta \int_{0}^{1} H(v(t)) d t
$$

The circle acts on $\mathscr{L}$ by rotation of the domain, that is,

$$
r_{*}(v)(t)=v(t+r), \quad v \in \mathscr{L}, r, t \in S^{1} .
$$

This action extends to an action on $\mathscr{L} \times \mathbb{R}$, where the circle acts trivially on the second factor. The Rabinowitz action functional is invariant under this circle action. In particular, its critical set is invariant, too. Hence we cannot expect the Rabinowitz action functional to be Morse. However, we show in this section that generically the Rabinowitz action functional is Morse-Bott. Here a function $f$ is called Morse-Bott if its set $C$ of critical points is a submanifold (possibly with components of different dimensions), and for every $x \in C$ the kernel of the Hessian of $f$ at $x$ equals $T_{x} C$.

A classical theorem of A. Wasserman [1969] asserts that if $G$ is a compact Lie group, then on finite dimensional $G$-manifolds the critical set of a generic $G$ invariant function consists of nondegenerate isolated $G$-orbits. The aim of this section is to prove a statement similar to Wasserman's theorem for the Rabinowitz action functional. Namely, we show that generically the fixed part of the critical set is a connected Morse-Bott component and away from the fixed part the assertion of Wasserman's Theorem holds true. Note that in the case of the Rabinowitz action functional the fixed part of the $S^{1}$-action on the critical set corresponds to the constant solutions on $H^{-1}(0)$ with Lagrange multiplier 0 , and the part of the critical set on which the circle acts with finite isotropy corresponds to the nonconstant Reeb orbits.

Theorem B.1. There exists a subset $u \subset C_{c}^{\infty}(V)$ of the second category, such that for every $H \in \mathcal{U}$ the Rabinowitz action functional $\mathscr{A}^{H}$ is Morse-Bott and its critical set consists of $H^{-1}(0)$ together with a disjoint union of circles.

Proof. We prove the theorem in four steps. For $k \in \mathbb{N}$ we denote by $C_{c}^{k}(V)$ the vector space of $C^{k}$-functions on $V$ of class $C^{k}$.

Step 1. For each $n \leq k \leq \infty$ denote by $u_{1}^{k}$ the subset of $C_{c}^{k}(V)$ consisting of $H \in C_{c}^{k}(V)$ with the property that 0 is a regular value of $H$. Then $u_{1}^{k}$ is an open and dense subset of $C_{c}^{k}(V)$.

Pick $H \in C_{c}^{k}(V)$. We first show that $H$ can be approximated by elements in $u_{1}^{k}$. By Sard's theorem for each $H \in C_{c}^{k}(V)$ the set $\mathscr{R}(H) \subset \mathbb{R}$ of regular values 
of $H$ is dense. Hence we can choose a sequence $c_{v} \in \mathscr{R}(H)$ converging to 0 . It follows that $H+c_{\nu}$ is a sequence in $u_{1}^{k}$ which converges to $H$. Hence $u_{1}^{k}$ is dense in $C_{c}^{k}(V)$. Moreover, since $H^{-1}(0)$ is compact for every $H \in u_{1}^{k}$ it follows that $u_{1}^{k}$ is also open in $C_{c}^{k}(V)$.

Step 2. For each $n \leq k<\infty$ there exists a subset $u_{2}^{k}$ of $u_{1}^{k}$ of second category such that for every $H \in u_{2}^{k}$ all nontrivial Reeb orbits are isolated and nondegenerate.

Consider the Hilbert manifold

$$
\mathscr{H}=W^{1,2}\left(S^{1}, V\right) \times \mathbb{R}
$$

where we define $W^{1,2}(V)$ by embedding $V$ into $\mathbb{R}^{N}$ for $N$ large enough. Over the Hilbert manifold $\mathscr{H}$ we introduce the Hilbert bundle

$$
\pi: \mathscr{E} \rightarrow \mathscr{H}
$$

whose fiber over $(v, \eta) \in \mathscr{H}$ is given by

$$
\mathscr{E}_{(v, \eta)}=L^{2}\left(S^{1}, v^{*} T V\right) \times \mathbb{R} .
$$

For $H \in C_{c}^{k}(V)$ we define a section

$$
s_{H}: \mathscr{H} \rightarrow \mathscr{E}
$$

by

$$
s_{H}(w)=\nabla \mathscr{A}^{H}(w) .
$$

Note that the zero set $s_{H}^{-1}(0)$ coincides with the critical points of the Rabinowitz action functional. If $w \in \mathscr{H} \subset \mathscr{E}$, then there is a canonical splitting

$$
T_{w} \mathscr{E}=\mathscr{E}_{w} \times T_{w} \mathscr{H} .
$$

We denote by

$$
\Pi_{w}: T_{w} \mathscr{E} \rightarrow \mathscr{E}_{w}
$$

the projection along $T_{w} \mathscr{H}$. If $w \in s_{H}^{-1}(0)$ the vertical differential

$$
D s_{H}(w): T_{w} \mathscr{H} \rightarrow \mathscr{E} w
$$

is given by

$$
D s_{H}(w)=\Pi_{w} \circ d s_{H}(w) .
$$

Note that it coincides with the Hessian of the Rabinowitz action functional at $w$. If $w=(v, \eta)$ is a critical point of $\mathscr{A}^{H}$, then it follows from the $S^{1}$-invariance of $\mathscr{A}^{H}$ that the infinitesimal generator

$$
R=\left(\partial_{t} v, 0\right)
$$

of the $S^{1}$-action satisfies

$$
R \in \operatorname{coker}\left(D s_{H}(w)\right) .
$$


We introduce the following quotient space of $\mathscr{E}^{\mathscr{w}} \mathrm{w}$

$$
\mathscr{F}_{w}=\mathscr{E}_{w} /\langle R\rangle .
$$

We denote by

$$
\overline{\mathbb{D}} s_{H}(w): T_{w} \mathscr{H} \rightarrow \mathscr{F}_{w}
$$

the linear map introduced from the vertical differential.

We extend the section $s_{H}$ to a larger space by considering the section

$$
S: u_{1}^{k} \times \mathscr{H} \rightarrow \mathscr{E}
$$

which is defined by

$$
S(H, w)=s_{H}(w), \quad H \in u_{1}^{k}, w \in \mathcal{H} .
$$

If $(H, w) \in S^{-1}(0)$ with $w=(v, \eta)$, then the vertical differential

$$
D S(H, w): T_{(H, w)}\left(u_{1}^{k} \times \mathscr{H}\right)=C_{c}^{k}(V) \times T_{w} \mathscr{H} \rightarrow \mathscr{E}_{w}
$$

is given for $\hat{H} \in C_{c}^{k}(V)$ and $\hat{w} \in T_{w} \mathscr{H}$ by

$$
D S(H, w)(\hat{H}, \hat{w})=D s_{H}(w) \hat{w}+\left(\begin{array}{c}
-\nabla \hat{H}(v) \\
0
\end{array}\right) .
$$

As before we denote by

$$
\overline{\mathbb{D}} S(H, w): T_{(H, w)}\left(u_{1}^{k} \times \mathscr{H}\right) \rightarrow \mathscr{F}_{w}
$$

the linear map induced from $D S(H, w)$.

The crucial point to establish Step 2 is the following claim.

Claim. If $(H, w) \in S^{-1}(0)$ and $w$ is not a fixed point of the $S^{1}$-action, then $\overline{\mathbb{D}} S(H, w)$ is surjective.

Since $\overline{\mathbb{D}} s_{H}(w)$ is Fredholm, the image of $\overline{\mathbb{D}} S(H, w)$ is closed. Hence to show surjectivity, it suffices to prove that the orthogonal complement of the image of $\overline{\mathbb{D}} S(H, w)$ vanishes. To see that, pick

$$
x=(y, \zeta) \in \operatorname{im} \overline{\mathbb{D}} S(H, w)^{\perp} .
$$

It follows from (67) that

$$
\left\{\begin{array}{cl}
\left\langle\overline{\mathbb{D}} s_{H}(w) \hat{w}, x\right\rangle=0, & \forall \hat{w} \in T_{w} \mathscr{H} \\
\langle\nabla \hat{H}(v), y\rangle=0, \quad \forall \hat{H} \in C_{c}^{k}(V) .
\end{array}\right.
$$

The first equation in (68) implies that

$$
x \in \operatorname{ker}\left(\overline{\mathbb{D}} s_{H}(w)\right)^{*}
$$


which shows that each representative $z \in L^{2}\left(S^{1}, v^{*} T V\right)$ of $y$ is of class $C^{k-1}$. Pick such a representative $z$ of $y$ and assume by contradiction that there exists $t_{0} \in S^{1}$ such that

$$
z\left(t_{0}\right) \notin\left\langle\partial_{t} v\left(t_{0}\right)\right\rangle .
$$

Choose $C^{k}$-coordinates around $v\left(t_{0}\right)$ of the following form. For $\epsilon>0$ there is a $C^{k-1}$-map

$$
\psi: Q=(-\epsilon, \epsilon)^{n} \rightarrow V
$$

satisfying

$$
\left\{\begin{array}{l}
\psi\left(r_{1}, 0\right)=v\left(t_{0}+r_{1}\right), r_{1} \in(-\epsilon, \epsilon) \\
\partial_{2} \psi(0)=z\left(t_{0}\right) .
\end{array}\right.
$$

Choose compactly supported functions $\alpha, \beta \in C_{0}^{k}((-\epsilon, \epsilon))$ such that

$$
\alpha(0)=1, \quad \beta(0)=0, \quad \beta^{\prime}(0)=1 .
$$

We introduce the function $h \in C_{0}^{k}(Q)$ by

$$
h\left(r_{1}, r_{2}, r\right)=\alpha\left(r_{1}\right) \cdot \beta\left(r_{2}\right), \quad r_{1}, r_{2} \in(-\epsilon, \epsilon), r \in(-\epsilon, \epsilon)^{n-2} .
$$

Now define $\hat{H} \in C_{c}^{k}(V)$ by

$$
\hat{H}(v)=\left\{\begin{array}{cc}
h\left(\psi^{-1}(v)\right), & v \in \psi(Q) \\
0 & v \notin \psi(Q) .
\end{array}\right.
$$

The function $\hat{H}$ has the properties

$$
\left\{\begin{array}{c}
d \hat{H}\left(v\left(t_{0}\right)\right)\left(z\left(t_{0}\right)\right)=1 \\
d \hat{H}(v(t)) \partial_{t} v=0, \quad t \in S^{1} .
\end{array}\right.
$$

Moreover, since $z$ is continuous, as we noted above, we can achieve by letting $\alpha$ decay fast enough that in addition the following property holds:

$$
d \hat{H}(v(t))(z(t)) \geq 0, \quad t \in S^{1} .
$$

We further note that the second condition in (69) implies that if $\tilde{z}$ is another representative for $y$, then

$$
d \hat{H}(v(t))(\tilde{z}(t))=d \hat{H}(v(t))(z(t)) .
$$

We deduce from equations (69), (70), and (71) that

$$
\langle\nabla \hat{H}, y\rangle=\inf _{\substack{z \in W^{1,2}\left(S^{1}, v^{*} T V\right) \\[z]=y}} \int_{0}^{1} d \hat{H}(v(t))(z(t)) d t>0
$$

contradicting the second equation in (68). We conclude that $y=0$. 
To show that $x$ vanishes it remains to argue that $\zeta$ vanishes. To see that we write

$$
\hat{w}=(\hat{v}, \hat{\eta}) \in T_{w} \mathscr{H}=W^{1,2}\left(S^{1}, v^{*} T V\right) \times \mathbb{R} .
$$

Since $y=0$ the first equation in (68) becomes

$$
\zeta \int_{0}^{1} d H(v(t))(\hat{v}(t)) d t=0, \quad \forall \hat{v} \in W^{1,2}\left(S^{1}, v^{*} T V\right) .
$$

Note that $(v, \eta)$ is a critical point of the Rabinowitz action functional, hence $v$ is contained in $H^{-1}(0)$. Since $H \in u_{1}^{k}$ it follows that 0 is a regular value of $H$. Hence $d H(v(t))$ does not vanish identically and we conclude that $\zeta=0$. Hence $x=(y, \zeta)$ vanishes and this finishes the proof of the claim.

Step 2 follows from the claim by a standard argument: The zero set $\mathscr{Z}=$ $S^{-1}(0) \subset U_{1}^{k} \times \mathscr{H}$ is a Hilbert manifold and the projection onto the second factor induces a Fredholm map $\pi: \mathscr{E} \rightarrow u_{1}^{k}$. By the Sard-Smale theorem the set $u_{2}^{k} \subset u_{1}^{k}$ of regular values of $\pi$ is of second category and satisfies the properties in Step 2.

Step 3. There exists a subset $u_{2}^{\infty}$ of $u_{1}^{\infty}$ of second category such that for every $H \in U_{2}^{\infty}$ all nontrivial Reeb orbits are isolated and nondegenerate.

Step 3 follows from Step 2 by an argument due to Taubes. Choose $T>0$ and abbreviate

$$
\operatorname{crit}_{T}\left(\mathscr{A}^{H}\right)=\left\{(v, \eta) \in \operatorname{crit}\left(\mathscr{A}^{H}\right):|\eta| \in(0, T]\right\} .
$$

It follows from the Arzela-Ascoli theorem that the set crit $\left.T^{(} \mathscr{A}^{H}\right)$ is compact. For $n \leq k \leq \infty$ we abbreviate

$$
u_{2}^{k}(T)=\left\{H \in u_{1}^{k}: \overline{\mathbb{D}} s_{H}(w) \text { surjective for all } w \in \operatorname{crit}_{T}\left(\mathscr{A}^{H}\right)\right\} .
$$

Since $\operatorname{crit}_{T}\left(\mathscr{A}^{H}\right)$ is compact it follows that $u_{2}^{k}(T)$ is open in $u_{1}^{k}(T)$. Moreover, it follows from Step 2 that if $k<\infty$ it is also dense in $u_{1}^{k}$. Since $C^{\infty}$ is dense in $C^{k}$ for every $k$, a diagonal argument shows that $u_{2}^{\infty}(T)$ is also dense in $u_{1}^{\infty}$. It follows that

$$
u_{2}^{\infty}=\bigcap_{T \in \mathbb{N}} u_{2}^{\infty}(T)
$$

is of second category in $u_{1}^{\infty}$. This finishes the proof of Step 3.

Step 4. $U=U_{2}^{\infty}$ meets the requirements of the theorem.

By Step $1, u_{1}^{\infty}$ is open and dense in $C_{c}^{\infty}(V)$ and by Step 3, $u$ is of second category in $u_{1}^{\infty}$. It follows that $U$ is of second category in $C_{c}^{\infty}(V)$. Moreover, by definition for each $H \in U$ the Rabinowitz action functional $\mathscr{A}^{H}$ is Morse-Bott at the nontrivial Reeb orbits and these are isolated. Hence, the only point to verify, 
is that $\mathscr{A}^{H}$ is also Morse-Bott at the trivial Reeb orbits. Hence let $(v, 0)$ with $v \in H^{-1}(0)$ be a trivial Reeb orbit. In this case the kernel of the Hessian

$$
\operatorname{Hess}_{\nexists{ } H}(v, 0): W^{1,2}\left(S^{1}, T_{v} V\right) \times \mathbb{R} \rightarrow L^{2}\left(S^{1}, T_{v} V\right) \times \mathbb{R}
$$

is given by solutions $\hat{v} \in W^{1,2}\left(S^{1}, T_{v} V\right)$ and $\hat{\eta} \in \mathbb{R}$ of the problem

$$
\left\{\begin{array}{c}
\partial_{t} \hat{v}(t)=\hat{\eta} X_{H}(v), \quad t \in S^{1} \\
\int_{0}^{1} d H(v) \hat{v}(t) d t=0
\end{array}\right.
$$

Since $H \in \mathcal{U}_{1}^{\infty}$ and $v \in H^{-1}(0)$ it follows that $X_{H}(v) \neq 0$. Hence it follows from the first equation in (72) that

$$
\hat{\eta}=0, \quad \partial_{t} \hat{v}=0, t \in S^{1} .
$$

Now the second equation in (72) implies that

$$
\hat{v} \in \operatorname{ker}(d H(v))=T_{v} H^{-1}(0) .
$$

Consequently, the Rabinowitz action functional $\mathscr{A}^{H}$ is also Morse-Bott at the trivial Reeb orbits. This finishes the proof of Step 4 and hence of Theorem B.1.

\section{Appendix C: Spectral flow}

We compare in this appendix two spectral flows which appear in Lagrange multiplier type problems. To motivate this we first consider the Lagrange multiplier functional in finite dimensions. Let $(M, g)$ be a Riemannian manifold and $(V,\langle\cdot, \cdot\rangle)$ be a Euclidean vector space. For functions $f \in C^{\infty}(M)$ and $h \in$ $C^{\infty}(M, V)$ the Lagrange multiplier functional $F \in C^{\infty}(M \times V)$ is given by

$$
F(x, v)=f(x)+\langle v, h(x)\rangle .
$$

For $v \in V$ we denote by $F_{v} \in C^{\infty}(M)$ the function given by

$$
F_{v}=F(\cdot, v) .
$$

The Hessian of $F$ with respect to the metric $g \oplus g_{V}$ on $M \times V$, where $g_{V}=\langle\cdot, \cdot\rangle$ is the Euclidean scalar product on $V$, is given by

$$
\operatorname{Hess}_{F}(x, v)=\left(\begin{array}{cc}
\operatorname{Hess}_{F}(x) & d h(x)^{*} \\
d h(x) & 0
\end{array}\right) .
$$

Here the adjoint of $d h(x)$ is taken with respect to the inner products on $T_{x} M$ and $T_{h(x)} V \cong V$ given by the metric $g$ and by $\langle\cdot, \cdot\rangle$.

We compare in this appendix the spectral flows of $\operatorname{Hess}_{F}$ and Hess $F_{v}$ for Lagrange multiplier functionals not necessarily defined on a finite dimensional manifold. We will apply this in the following way. For $F=\mathscr{A}^{H}$ the functional $F_{v}$ is 
the action functional of classical mechanics whose spectral flow can be computed via the Conley-Zehnder indices [Robbin and Salamon 1995].

To formulate our theorem we use the setup of Robbin and Salamon [1995]. Let $W$ and $H$ be separable real Hilbert spaces such that $W \subset H$ is dense and the inclusion is compact. Let $A: W \rightarrow H$ be a bounded linear operator (with respect to the norms on $W$ and $H$ ). Viewing $A$ as an unbounded operator on $H$ with domain $\operatorname{dom}(A)=W$, recall the following definitions (see for example [Kato 1976]). The adjoint operator

$$
A^{*}: \operatorname{dom}\left(A^{*}\right):=\left\{\left.v \in H||\langle v, A w\rangle_{H}|\leq C| w\right|_{H} \text { for all } w \in W\right\} \rightarrow H
$$

is defined by the equation

$$
\left\langle A^{*} v, w\right\rangle_{H}=\langle v, A w\rangle_{H} .
$$

$A$ is called symmetric if $W \subset \operatorname{dom}\left(A^{*}\right)$ and $\left.A^{*}\right|_{W}=A$, that is, $\left\langle A^{*} v, w\right\rangle_{H}=$ $\langle v, A w\rangle_{H}$ for all $v, w \in W$. $A$ is self-adjoint if it is symmetric and $\operatorname{dom}\left(A^{*}\right)=W$. The spectrum of $A$ is the set of all complex numbers $\lambda$ such that the operator

$$
A-\lambda \cdot \mathrm{id}: W \rightarrow H
$$

is not bijective. Denote by $\operatorname{ker}(A)$ and $\mathscr{R}(A)$ the kernel and range (= image) of $A$, respectively. Denote by $\mathscr{L}(W, H)$ the space of bounded linear operators and by

$$
\mathscr{S}(W, H) \subset \mathscr{L}(W, H)
$$

the subspace of self-adjoint operators. The following lemma clarifies these concepts.

Lemma C.1. Let $W \subset H$ be Hilbert spaces such that the inclusion is compact and let $A: W \rightarrow H$ be a symmetric bounded linear operator. Then the following are equivalent:

(i) There exists $\lambda \in \mathbb{R}$ such that $A-\lambda \cdot \mathrm{id}: W \rightarrow H$ is bijective.

(ii) $A$ is self-adjoint considered as an unbounded operator on $H$ with domain $\operatorname{dom}(A)=W$.

(iii) One of the defect indices $d^{ \pm}(A):=\operatorname{codim}\left(\mathscr{R}\left(A^{\mathbb{C}} \pm i \cdot \mathrm{id}\right), H^{\mathbb{C}}\right)$ is zero. Here $A^{\mathbb{C}}: W^{\mathbb{C}} \rightarrow H^{\mathbb{C}}$ denotes the complex linear extension of $A$ to the complexified Hilbert spaces.

(iv) The spectrum of $A$ is discrete and consists of real eigenvalues of finite multiplicity.

Proof. We first show that (i) $\Rightarrow$ (ii), that is, $\operatorname{dom}\left(A^{*}\right)=\operatorname{dom}(A)=W$. To see this let $v \in \operatorname{dom}\left(A^{*}\right)$. Since $A-\lambda \cdot \mathrm{id}$ is bijective and $A$ is symmetric, there exists 
$w \in W$ such that

$$
\left(A^{*}-\lambda \cdot \mathrm{id}\right) v=(A-\lambda \cdot \mathrm{id}) w=\left(A^{*}-\lambda \cdot \mathrm{id}\right) w,
$$

which implies

$$
(A-\lambda \cdot \mathrm{id})^{*}(v-w)=0 .
$$

Again using the fact that $A-\lambda \cdot \mathrm{id}$ is bijective, we conclude that $(A-\lambda \cdot \mathrm{id})^{*}$ is bijective and hence

$$
v=w \in W .
$$

It follows that $A$ is self-adjoint with $\operatorname{dom}(A)=W$.

If $A$ is self-adjoint, then both defect indices are zero (see for example [Kato 1976, Theorem V.3.16]) so that (ii) $\Rightarrow$ (iii).

We show that (iii) $\Rightarrow$ (iv). Assume that $d^{-}(A)$ is zero, that is, $A^{\mathbb{C}}-i \cdot \mathrm{id}: W^{\mathbb{C}} \rightarrow$ $H^{\mathbb{C}}$ is invertible. Since the inclusion $\iota: W \rightarrow H$ is compact, the operator

$$
R:=\iota \circ\left(A^{\mathbb{C}}-i \cdot \mathrm{id}\right)^{-1}: H^{\mathbb{C}} \rightarrow H^{\mathbb{C}}
$$

is compact. In particular, its spectrum $\sigma(R)$ consists of eigenvalues, the only accumulation point in $\sigma(R)$ is zero, and the eigenspace for each eigenvalue except zero is finite dimensional. Let $\zeta \in \mathbb{C} \backslash\{i\}$. Then the following relations hold for the ranges

$$
\mathscr{R}\left(A^{\mathbb{C}}-\zeta \cdot \mathrm{id}\right)=R^{-1} \mathscr{R}\left(R-\frac{1}{\zeta-i} \cdot \mathrm{id}\right)
$$

and the kernels

$$
\operatorname{ker}\left(A^{\mathbb{C}}-\zeta \cdot \mathrm{id}\right)=\operatorname{ker}\left(R-\frac{1}{\zeta-i} \cdot \mathrm{id}\right)
$$

In particular, we have a bijection

$$
\sigma(R) \backslash\{0\} \rightarrow \sigma\left(A^{\mathbb{C}}\right), \quad \mu \mapsto \frac{1}{\mu}+i
$$

between the spectra under which the corresponding eigenspaces do not change, that is, for every $\mu \in \sigma(R) \backslash\{0\}$ the eigenspaces satisfy

$$
E_{\mu}(R)=E_{1 / \mu+i}\left(A^{\mathbb{C}}\right) \subset H .
$$

We conclude that the spectrum of $A$ consists of discrete eigenvalues of finite multiplicity, which are real because $A$ is symmetric. A similar argument holds for the case that $d^{+}(A)$ is zero. This shows that (iii) implies (iv).

That (iv) $\Rightarrow$ (i) is obvious.

Assume in addition that $V$ is a finite dimensional Hilbert space. Let $A \in$ $\mathscr{S}(W, H)$ be as before and $B \in \mathscr{L}(V, H)$ be a bounded linear operator. We denote 
by $A_{B}: W \oplus V \rightarrow H \oplus V$ the bounded symmetric operator defined by

$$
A_{B}(w, v)=\left(A w+B v, B^{*} w\right) .
$$

Lemma C.2. The operator $A_{B}$ is in $\mathscr{Y}(W \oplus V, H \oplus V)$.

Proof. We have to show that $A_{B}$ is self-adjoint. This is true if $B=0$. For arbitrary $B$ this follows from Theorem V.4.3 in [Kato 1976].

In the following orthogonality is always understood with respect to the inner product of $H$ and never with respect to the inner product of $W$.

Definition C.3. Let $A \in \mathscr{T}(W, H)$, and $B \in \mathscr{L}(V, H)$. We say that the tuple $(A, B)$ is regular if:

(i) $B$ is injective and $\mathscr{R}(B) \subset W$.

(ii) A maps $\mathscr{R}(B)$ to itself and the restriction $\hat{A}:=\left.A\right|_{\mathscr{R}(B)}$ is bijective.

A regular pair $(A, B)$ gives rise to the symmetric operator

$$
S_{A, B}:=B^{*} \hat{A}^{-1} B
$$

on $V$ whose signature we denote by

$$
\sigma(A, B)=\operatorname{sign}\left(S_{A, B}\right) .
$$

We now consider maps $A: \mathbb{R} \rightarrow \mathscr{Y}(W, H)$ and $B: \mathbb{R} \rightarrow \mathscr{L}(V, H)$ which are continuous with respect to the norm topology such that the limits

$$
\lim _{s \rightarrow \pm \infty} A(s)=A^{ \pm}, \quad \lim _{s \rightarrow \pm \infty} B(s)=B^{ \pm}
$$

exist and $A^{ \pm} \in \mathscr{Y}(W, H)$. We then define the map $A_{B}: \mathbb{R} \rightarrow \mathscr{Y}(W \oplus V, H \oplus V)$ by

Denote by

$$
A_{B}(s):=A(s)_{B(s)}, \quad s \in \mathbb{R} .
$$

$$
\mathscr{A}=\mathscr{A}(\mathbb{R}, W, H)
$$

the space of maps $A: \mathbb{R} \rightarrow \mathscr{S}(W, H)$ as above, which in addition satisfy that $A^{ \pm}$is bijective. Recall the following theorem of Robbin and Salamon about the existence of the spectral flow [Robbin and Salamon 1995, Theorem 4.23].

Theorem C.4. There exist unique maps $\mu: \mathcal{A}(\mathbb{R}, W, H) \rightarrow \mathbb{Z}$, one for every compact dense injection of Hilbert spaces $W \hookrightarrow H$, satisfying the following axioms.

(1) (Homotopy) $\mu$ is constant on connected components of $\mathscr{A}(\mathbb{R}, W, H)$.

(2) (Constant) If $A$ is constant, then $\mu(A)=0$.

(3) (Direct sum) $\mu\left(A_{1} \oplus A_{2}\right)=\mu\left(A_{1}\right)+\mu\left(A_{2}\right)$.

(4) (Normalization) For $W=H=\mathbb{R}$ and $A(t)=\arctan (t)$, we have $\mu(A)=1$. 
The number $\mu(A)$ is called the spectral flow of $A$.

These axioms easily imply the following generalization of the normalization axiom:

- (Crossing) For $W=H$ finite dimensional,

$$
\mu(A)=\frac{1}{2}\left(\operatorname{sign}\left(A^{+}\right)-\operatorname{sign}\left(A^{-}\right)\right) .
$$

To define the spectral flow also for maps $A$ whose limits $A^{ \pm}$are not necessarily bijective we choose a smooth cutoff function $\beta \in C^{\infty}(\mathbb{R},[-1,1])$ such that $\beta(s)=1$ for $s \geq 1$ and $\beta(s)=-1$ for $s \leq-1$ and define

$$
A_{\delta}(s):=A(s)-\delta \beta(s) \text { id, } \quad \mu(A):=\lim _{\delta \searrow 0} \mu\left(A_{\delta}\right) .
$$

Note that the limit in $\mu(A)$ stabilizes for sufficiently small $\delta>0$.

Theorem C.5. Let $A: \mathbb{R} \rightarrow \mathscr{Y}(W, H)$ and $B: \mathbb{R} \rightarrow \mathscr{L}(V, H)$ be continuous maps whose limits $\lim _{s \rightarrow \pm \infty} A(s)=A^{ \pm}$and $\lim _{s \rightarrow \pm \infty} B(s)=B^{ \pm}$exist. Assume moreover that $\left(A^{ \pm}, B^{ \pm}\right)$are regular pairs. Then the spectral flows of $A$ and $A_{B}$ are related by

$$
\mu\left(A_{B}\right)=\mu(A)+\frac{1}{2}\left(\sigma\left(A^{-}, B^{-}\right)-\sigma\left(A^{+}, B^{+}\right)\right) .
$$

Proof. Choose two cutoff functions $\beta^{ \pm} \in C^{\infty}(\mathbb{R},[0,1])$ with the property that $\beta^{+}(s)=1$ for $s \geq 1, \beta^{+}(s)=0$ for $s \leq 0, \beta^{-}(s)=1$ for $s \leq-1$, and $\beta^{-}(s)=0$ for $s \geq 0$. Define $S_{A, B} \in \mathscr{A}(\mathbb{R}, V, V)$ by

$$
S_{A, B}(s)=\beta^{+}(s) S_{A^{+}, B^{+}}+\beta^{-}(s) S_{A^{-}, B^{-}} .
$$

Abbreviate $P_{V}: H \oplus V \rightarrow V$ the canonical projection. We prove the theorem in three steps.

Step 1. If $\delta>0$ is small enough then $\left(A_{B}\right)_{\delta}$ is homotopic in $\mathcal{A}(\mathbb{R}, W, H)$ to $\left(A_{\delta}\right)_{B}-\delta P_{V}^{*} S_{A, B} P_{V}$.

To see this, we first compute

$$
\begin{aligned}
\left(A_{B}\right)_{\delta}(w, v) & =\left(A w+B v-\delta \beta w, B^{*} w-\delta \beta v\right), \\
\left(A_{\delta}\right)_{B}(w, v) & =\left(A w+B v-\delta \beta w, B^{*} w\right), \\
\left(A_{B}\right)_{\delta}(w, v)-\left(A_{\delta}\right)_{B}(w, v) & =(0,-\delta \beta v),
\end{aligned}
$$

thus $\left(A_{B}\right)_{\delta}=\left(A_{\delta}\right)_{B}-\delta \beta P_{V}^{*} P_{V}$. We will show that for $\delta>0$ sufficiently small and symmetric linear maps $S^{ \pm} \in \mathscr{L}(V)$ whose norm is small enough the operators $\left(A_{\delta}^{ \pm}\right)_{B^{ \pm}}+P_{V}^{*} S^{ \pm} P_{V}$ are bijective; applying this to $S^{ \pm}=-(1-t) \delta \cdot \mathrm{id}-t \delta S_{A, b}^{ \pm}$ for $t \in[0,1]$ then proves Step 1 . 
By Theorem V.4.3 in [Kato 1976] the operators $\left(A_{\delta}^{ \pm}\right)_{B^{ \pm}}+P_{V}^{*} S^{ \pm} P_{V}$ are selfadjoint with dense domain $W \oplus V$, hence by Lemma C.1 their spectrum consists of eigenvalues. Thus it suffices to show injectivity. Let $(w, v) \in W \times V$ be in the kernel of $\left(A_{\delta}^{ \pm}\right)_{B^{ \pm}}+P_{V}^{*} S^{ \pm} P_{V}$. Then $(w, v)$ solves

$$
\left\{\begin{array}{c}
\left(A^{ \pm} \mp \delta \cdot \mathrm{id}\right) w+B^{ \pm} v=0 \\
\left(B^{ \pm}\right)^{*} w+S^{ \pm} v=0
\end{array}\right.
$$

which is equivalent to

$$
\left\{\begin{array}{c}
w=-\left(A^{ \pm} \mp \delta \cdot \mathrm{id}\right)^{-1} B^{ \pm} v \\
-\left(B^{ \pm}\right)^{*}\left(A^{ \pm} \mp \delta \cdot \mathrm{id}\right)^{-1} B^{ \pm} v+S^{ \pm} v=0 .
\end{array}\right.
$$

But $\left(B^{ \pm}\right)^{*}\left(A^{ \pm}-\delta \cdot \mathrm{id}\right)^{-1} B^{ \pm}$converges to the nondegenerate linear map $S_{A^{ \pm}, B^{ \pm}}$ as $\delta$ goes to zero, and hence the second equation in (76) has only the trivial solution $v=0$ if the norm of $S^{ \pm}$is small enough and hence $(w, v)=(0,0)$. This shows injectivity and hence the assertion of Step 1 follows.

Step 2. For $\delta>0$ small enough and $\epsilon \in[0,1]$ the maps $\left(A_{\delta}\right)_{\epsilon B}-\delta P_{V}^{*} S_{A, B} P_{V}$ are in $A(\mathbb{R}, W \oplus V, H \oplus V)$, that is, their asymptotics are bijective.

Step 2 follows by a similar reasoning as Step 1. Assume that $(w, v) \in W \oplus V$ lies in the kernel of one of the asymptotic operators. Then $(w, v)$ solves

$$
\left\{\begin{array}{c}
w=-\epsilon\left(A^{ \pm} \mp \delta \cdot \mathrm{id}\right)^{-1} B^{ \pm} v \\
-\epsilon^{2}\left(B^{ \pm}\right)^{*}\left(A^{ \pm} \mp \delta \cdot \mathrm{id}\right)^{-1} B^{ \pm} v-\delta S_{A^{ \pm}, B^{ \pm}} v=0 .
\end{array}\right.
$$

The second equation has the form

$$
-\left(\epsilon^{2}+\delta\right) C_{\delta} v+D_{\delta} v=0
$$

with $C_{\delta}=\left(B^{ \pm}\right)^{*}\left(A^{ \pm} \mp \delta \cdot \mathrm{id}\right)^{-1} B^{ \pm}$, which is invertible for all sufficiently small $\delta$, and an operator $D_{\delta}$ of order $\delta^{2}$. For $\epsilon \in[0,1]$ and sufficiently small $\delta_{0}$ this equation has only the trivial solution $v=0$, thus $(w, v)=(0,0)$ and Step 2 follows.

Step 3. We prove the theorem.

Using the properties of the spectral flow from Theorem C.4 we are now in position to compute

$$
\begin{aligned}
\mu\left(\left(A_{B}\right)_{\delta}\right) & =\mu\left(\left(A_{\delta}\right)_{B}-\delta P_{V}^{*} S_{A, B} P_{V}\right)=\mu\left(\left(A_{\delta}\right)_{0}-\delta P_{V}^{*} S_{A, B} P_{V}\right) \\
& =\mu\left(A_{\delta} \oplus-\delta S_{A, B}\right)=\mu\left(A_{\delta}\right)+\mu\left(-\delta S_{A, B}\right) \\
& =\mu\left(A_{\delta}\right)+\frac{1}{2}\left(\sigma\left(A^{-}, B^{-}\right)-\sigma\left(A^{+}, B^{+}\right)\right) .
\end{aligned}
$$

Here we have used Step 1 for the first equality, Step 2 for the second one, and the (crossing) property of $\mu$ for the last one. Taking the limit $\delta \searrow 0$ the theorem follows. 
There are scenarios where the signature $\sigma(A, B)$ can easily be computed. We formulate such an example for a Lagrange multiplier functional on a finite dimensional manifold which can easily be generalized to infinite dimensional cases.

Lemma C.6. Let $(M, g)$ be a Riemannian manifold and let $f, h \in C^{\infty}(M)$ be such that 0 is a regular value of $h$. Let $\left(x_{0}, v_{0}\right)$ be a critical point of the Lagrange multiplier functional $F \in C^{\infty}(M \times \mathbb{R})$ given by $F(x, v)=f(x)+v h(x)$. Assume that there exists $\epsilon>0$ and a smooth curve $(x, v) \in C^{\infty}((-\epsilon, \epsilon), M \times \mathbb{R})$ satisfying $(x(0), v(0))=\left(x_{0}, v_{0}\right)$ such that the following holds:

(i) $\partial_{\rho} x(0)=\nabla h\left(x_{0}\right)$.

(ii) $\partial_{\rho} v(0) \neq 0$.

(iii) $(x(\rho), v(\rho))$ for $\rho \in(-\epsilon, \epsilon)$ is a critical point of the Lagrange multiplier functional $F^{\rho} \in C^{\infty}(M \times \mathbb{R})$ given by $F^{\rho}(x, v):=f(x)+v \cdot(h(x)-\rho)$.

Then the pair $\left(\operatorname{Hess}_{F_{v_{0}}}\left(x_{0}\right), \nabla h\left(x_{0}\right)\right)$ is regular in the sense of Definition C.3 and its signature is

$$
\sigma\left(\operatorname{Hess}_{F_{v_{0}}}\left(x_{0}\right), \nabla h\left(x_{0}\right)\right)=-\operatorname{sign}\left(\partial_{\rho} v(0)\right) .
$$

Proof. The identity $d F^{\rho}(x(\rho), v(\rho))=0$ for $\rho \in(-\epsilon, \epsilon)$ is equivalent to

$$
\left\{\begin{array}{c}
d f(x(\rho))+v(\rho) \cdot d h(x(\rho))=0 \\
h(x(\rho))=\rho .
\end{array}\right.
$$

The first equation in (77) can be written as

$$
\nabla f(x(\rho))+v(\rho) \cdot \nabla h(x(\rho))=0 .
$$

Differentiating this identity with respect to $\rho$ and evaluating at $\rho=0$ we compute using assumption (i)

$$
\begin{aligned}
0 & =\operatorname{Hess}_{f}\left(x_{0}\right) \partial_{\rho} x(0)+v_{0} \operatorname{Hess}_{h}\left(x_{0}\right) \partial_{\rho} x(0)+\partial_{\rho} v(0) \nabla h\left(x_{0}\right) \\
& =\operatorname{Hess}_{F_{v_{0}}}\left(x_{0}\right) \nabla h\left(x_{0}\right)+\partial_{\rho} v(0) \nabla h\left(x_{0}\right) .
\end{aligned}
$$

More specifically, $\nabla h\left(x_{0}\right)$ is an eigenvector of $\operatorname{Hess}_{F_{v_{0}}}$ to the nonzero eigenvalue $-\partial_{\rho} v(0)$.

It is now straightforward to check that the pair $\left(\operatorname{Hess}_{F_{v_{0}}}\left(x_{0}\right), \nabla h\left(x_{0}\right)\right)$ is regular. Condition (i) in Definition C.3 follows from the assumption that 0 is a regular value of $h$ and thus $\nabla h\left(x_{0}\right) \neq 0$. Since $\nabla h\left(x_{0}\right)$ is an eigenvector of the Hessian to a nonzero eigenvalue, condition (ii) is satisfied as well. 
To compute the signature we calculate

$$
\begin{aligned}
\sigma\left(\operatorname{Hess}_{F_{v_{0}}}\left(x_{0}\right), \nabla h\left(x_{0}\right)\right) & =\operatorname{sign}\left(d h\left(x_{0}\right) \widehat{\operatorname{Hess}_{F}} F_{v_{0}}\left(x_{0}\right)^{-1} \nabla h\left(x_{0}\right)\right) \\
& =\operatorname{sign}\left(-\frac{\left\|\nabla h\left(x_{0}\right)\right\|^{2}}{\partial_{\rho} v(0)}\right)=-\operatorname{sign}\left(\partial_{\rho} v(0)\right) .
\end{aligned}
$$

\section{Appendix D: Some topological obstructions}

During the first author's talk at the Workshop on Symplectic Geometry, Contact Geometry and Interactions in Lille 2007, E. Giroux suggested that in the case $n=2$, Corollary 1.7 results from the following topological fact.

Lemma D.1. There exists no smooth embedding of $S^{*} S^{2} \cong \mathbb{R} P^{3}$ into a subcritical Stein surface.

Right after the talk, participants suggested the following three proofs of this fact. Note that every subcritical Stein surface is $\mathbb{C}^{2}$ or a boundary connected sum of copies of $S^{1} \times \mathbb{R}^{3}$, which embeds smoothly into $\mathbb{R}^{4}$; thus for the lemma it suffices to prove that $\mathbb{R} P^{3}$ admits no embedding into $\mathbb{R}^{4}$.

Proof 1 (V. Kharlamov). This proof is based on the following theorem of Whitney (see for example [Massey 1969]): The Euler number $e(\Sigma) \in \mathbb{Z}$ of the normal bundle of a closed connected nonorientable surface $\Sigma$ embedded in $\mathbb{R}^{4}$ satisfies $e(\Sigma) \equiv$ $2 \chi(\Sigma) \bmod 4$. Now suppose we have an embedding $\mathbb{R} P^{3} \subset \mathbb{R}^{4}$. Then the normal Euler number of the linear subspace $\mathbb{R} P^{2} \subset \mathbb{R} P^{3} \subset \mathbb{R}^{4}$ satisfies $e\left(\mathbb{R} P^{2}\right) \equiv 2 \bmod$ 4. But a nonvanishing normal vector field to $\mathbb{R} P^{3}$ in $\mathbb{R}^{4}$ (which exists because $\mathbb{R} P^{3}$ is orientable) provides a nonvanishing section of the normal bundle of $\mathbb{R} P^{2} \subset \mathbb{R}^{4}$, contradicting nontriviality of $e\left(\mathbb{R} P^{2}\right)$.

Proof 2 (T. Ekholm). This proof is based on a theorem of Ekholm [2001]: The Euler characteristic of the (resolved) self-intersection surface of a generic immersion of $S^{3}$ to $\mathbb{R}^{4}$ has the same parity as the number of quadruple points. Now suppose we have an embedding $\mathbb{R} P^{3} \subset \mathbb{R}^{4}$. Composition with the covering $S^{3} \rightarrow \mathbb{R} P^{3}$ yields an immersion of $S^{3}$ to $\mathbb{R}^{4}$ which can be perturbed (via a normal vector field to $\mathbb{R} P^{3}$ vanishing along $\mathbb{R} P^{2}$ ) to have self-intersection surface $\mathbb{R} P^{2}$ and no quadruple points, contradicting Ekholm's theorem.

The third proof, suggested by P. Lisca, yields in fact the following more general result:

Proposition D.2. For $n \geq 2$ even there exists no smooth embedding of $S^{*} S^{n}$ into a subcritical Stein 2 n-manifold.

Proof 3 (P. Lisca). Suppose we have an embedding $S^{*} S^{n} \cong \Sigma \subset V$ into a subcritical Stein $2 n$-manifold $V$ for $n \geq 2$ even. From $H_{2 n-1}(V ; \mathbb{Z})=0$ it follows that $\Sigma$ 
bounds a compact subset $B \subset V$. Denote by $C$ the closure of $V \backslash B$, so $V=B \cup_{\Sigma} C$. Since $n$ is even, the Gysin homology sequence of the sphere bundle $S^{*} S^{n} \rightarrow S^{n}$ shows $H_{n}\left(S^{*} S^{n} ; \mathbb{Z}\right)=0$ and $H_{n-1}\left(S^{*} S^{n} ; \mathbb{Z}\right)=\mathbb{Z}_{2}$. Using this and $H_{n}(V ; \mathbb{Z})=0$, the Mayer-Vietoris sequence for $V=B \cup_{\Sigma} C$ implies $H_{n}(B ; \mathbb{Z})=H_{n}(C ; \mathbb{Z})=0$. Let $X:=D^{*} S^{n} \cup_{\Sigma} B$ be the closed oriented $2 n$-manifold obtained by gluing the unit disk cotangent bundle $D^{*} S^{n}$ (with orientation reversed) to $B$ along $\Sigma$. Again by the Mayer-Vietoris sequence, we find that the free part $H_{n}(X ; \mathbb{Z}) /$ torsion is isomorphic to $\mathbb{Z}$ and the zero section $S^{n} \subset D^{*} S^{n}$ is a generator or twice a generator. But for $n$ even the zero section $S^{n}$ has self-intersection number 2 in $D^{*} S^{n}$, hence -2 in $X$. This is impossible if the zero section is twice a generator of $H_{n}(X ; \mathbb{Z}) /$ torsion, and if it is a generator this contradicts unimodularity of the intersection form (which is an immediate consequence of Poincaré duality).

Remark. The fact that $S^{*} S^{2}$ has no exact contact embedding into a subcritical Stein surface $V$ can also be proved symplectically as follows, using a deep result by Gromov about holomorphic fillings. First note that every subcritical Stein surface admits an exact symplectic embedding into $\mathbb{R}^{4}$. (A subcritical Stein domain of complex dimension 2 is either $B^{4}$ or the boundary connected sum of $k \geq 1$ copies of $S^{1} \times B^{3}$, which can be realized as a Stein domain in $\mathbb{C}^{2}$ using the techniques in [Eliashberg 1990]; see also [Gompf 2005].) Thus it suffices again to consider the case $V=\mathbb{R}^{4}$.

Suppose there exists an exact contact embedding $\iota: S^{*} S^{2} \hookrightarrow \mathbb{R}^{4}$. Removing the bounded component of $\mathbb{R}^{4} \backslash \iota\left(S^{*} S^{2}\right)$ and gluing in the unit ball bundle $D^{*} S^{2}$ yields an exact convex symplectic manifold $W$ which contains an embedded Lagrangian 2-sphere (the zero section in $D^{*} S^{2}$ ). On the other hand, $W$ is symplectomorphic to $\mathbb{R}^{4}$ outside compact set. So a result of Gromov [1985] implies that $W$ is in fact symplectomorphic to $\mathbb{R}^{4}$. But this is a contradiction because $\mathbb{R}^{4}$ does not admit any embedded Lagrangian 2-spheres.

Remark. We have not investigated obstructions to smooth embeddings of $S^{*} S^{n}$ into subcritical Stein manifolds for $n$ odd. As pointed out in the introduction, at least for $n=3$ and $n=7$ there are no obstructions and $S^{*} S^{n}$ embeds smoothly into $\mathbb{C}^{n}$.

\section{Appendix E: Cylindrical contact homology}

In this appendix we collect known results on the cylindrical contact homology of $\left(S^{*} S^{n}, \xi_{\mathrm{st}}\right)$ and the boundaries of subcritical Stein domains. In the latter case we have:

Theorem E.1 (M.-L. Yau [2004]). Let $V$ be a subcritical Stein domain of dimension $2 n \geq 4$ with $c_{1}(V)=0$, and let $\xi_{\text {subcrit }}$ be the contact structure on $\partial V$ 
given by the maximal complex subspaces. Then the cylindrical contact homology of $\left(\partial V, \xi_{\text {subcrit }}\right)$ is well-defined and given by

$$
H C_{*}^{\text {cyl }}\left(M, \xi_{\text {subcrit }}\right) \cong \bigoplus_{k \in \mathbb{N}} H_{n-1-*+2 k}(V ; \mathbb{Q})
$$

Here (and throughout this appendix) cylindrical contact is taken with $\mathbb{Q}$-coefficients and graded by Conley-Zehnder indices. Applying this result to the subcritical Stein domain $B^{n+1} \times S^{n-1}$ we find:

For $n$ even,

$$
H C_{*}^{\text {cyl }}\left(S^{n} \times S^{n-1}, \xi_{\text {subcrit }}\right) \cong \begin{cases}\mathbb{Q} & * \geq n+1 \text { odd } \\ \mathbb{Q} & * \geq 2 \text { even } \\ 0 & \text { else }\end{cases}
$$

For $n$ odd,

$$
H C_{*}^{\text {cyl }}\left(S^{n} \times S^{n-1}, \xi_{\text {subcrit }}\right) \cong \begin{cases}\mathbb{Q} \oplus \mathbb{Q} & * \geq n+1 \text { even, } \\ \mathbb{Q} & 2 \leq * \leq n-1 \text { even } \\ 0 & \text { else }\end{cases}
$$

In particular, for $n=3$ and $n=7$ we obtain:

$$
\begin{aligned}
H C_{*}^{\text {cyl }}\left(S^{3} \times S^{2}, \xi_{\text {subcrit }}\right) \cong \begin{cases}\mathbb{Q} \oplus \mathbb{Q} & * \geq 4 \text { even, } \\
\mathbb{Q} & *=2, \\
0 & \text { else, }\end{cases} \\
H C_{*}^{\text {cyl }}\left(S^{7} \times S^{6}, \xi_{\text {subcrit }}\right) \cong \begin{cases}\mathbb{Q} \oplus \mathbb{Q} & * \geq 8 \text { even, } \\
\mathbb{Q} & *=2,4,6, \\
0 & \text { else. }\end{cases}
\end{aligned}
$$

Next we consider the unit cotangent bundle $\left(S^{*} S^{n}, \xi_{\text {st }}\right)$ with its standard contact structure. As noted in Section 4, the Morse index of a $k$-fold covered closed geodesic for the round metric on $S^{n}$ equals $(2 k-1)(n-1)$. The following lemma is well known.

Lemma E.2. For each $k \in \mathbb{N}$ the space of $k$-fold covered (unparametrized) closed geodesics is diffeomorphic to the complex quadric $Q_{n-1} \subset \mathbb{C} P^{n}$ and has the following homology:

For $n$ even,

$$
H_{*}\left(Q_{n-1} ; \mathbb{Q}\right) \cong \begin{cases}\mathbb{Q} & 0 \leq * \leq 2(n-1) \text { even }, \\ 0 & \text { else. }\end{cases}
$$


For $n$ odd,

$$
H_{*}\left(Q_{n-1} ; \mathbb{Q}\right) \cong \begin{cases}\mathbb{Q} \oplus \mathbb{Q} \quad *=n-1, \\ \mathbb{Q} & 0 \leq * \leq 2(n-1) \text { even, } * \neq n-1, \\ 0 & \text { else. }\end{cases}
$$

By a Morse-Bott argument, the complex for cylindrical contact homology is generated by elements of degrees $(2 k-1)(n-1)+d_{j}, k \in \mathbb{N}$, where $d_{j}$ are the degrees of generators of $H_{*}\left(Q_{n-1} ; \mathbb{Q}\right)$. Since the homology of $Q_{n-1}$ lives in even degrees, it follows that all generators have odd (if $n$ is even) respectively even (if $n$ is odd) degrees. So the boundary operator is zero and cylindrical contact homology is freely generated by elements of degrees $(2 k-1)(n-1)+d_{j}, k \in \mathbb{N}$. Now a short computation yields:

For $n$ even,

$$
H C_{*}^{\mathrm{cyl}}\left(S^{*} S^{n}, \xi_{\mathrm{st}}\right) \cong \begin{cases}\mathbb{Q} \oplus \mathbb{Q} & *=k(n-1), k \geq 3 \text { odd }, \\ \mathbb{Q} & * \geq n-1 \text { odd }, * \neq k(n-1) \text { for odd } k \geq 3 \\ 0 & \text { else. }\end{cases}
$$

For $n$ odd,

$$
H C_{*}^{\text {cyl }}\left(S^{*} S^{n}, \xi_{\text {st }}\right) \cong \begin{cases}\mathbb{Q} \oplus \mathbb{Q} & *=k(n-1), k \geq 2, \\ \mathbb{Q} & * \geq n-1 \text { even, } * \neq k(n-1) \text { for } k \geq 2, \\ 0 & \text { else. }\end{cases}
$$

In particular, for $n=3$ and $n=7$ we obtain:

$$
\begin{aligned}
& H C_{*}^{\mathrm{cyl}}\left(S^{*} S^{3}, \xi_{\mathrm{st}}\right) \cong \begin{cases}\mathbb{Q} \oplus \mathbb{Q} & * \geq 4 \text { even, } \\
\mathbb{Q} & *=2, \\
0 & \text { else, }\end{cases} \\
& H C_{*}^{\mathrm{cyl}}\left(S^{*} S^{7}, \xi_{\mathrm{st}}\right) \cong \begin{cases}\mathbb{Q} \oplus \mathbb{Q} & *=6 k, k \geq 2, \\
\mathbb{Q} & * \geq 6 \text { even, } * \neq 6 k \text { for } k \geq 2, \\
0 & \text { else. }\end{cases}
\end{aligned}
$$

Together these computations show:

Corollary E.3. The contact structures $\xi_{\text {st }}$ and $\xi_{\text {subcrit }}$ on $S^{*} S^{3} \cong S^{3} \times S^{2}$ have isomorphic cylindrical contact homology, while the contact structures $\xi_{\mathrm{st}}$ and $\xi_{\text {subcrit }}$ on $S^{*} S^{7} \cong S^{7} \times S^{6}$ have nonisomorphic cylindrical contact homologies.

\section{Acknowledgement}

We thank O. van Koert for his remark that led to Appendix E. 


\section{References}

[Audin et al. 1994] M. Audin, F. Lalonde, and L. Polterovich, "Symplectic rigidity: Lagrangian submanifolds", pp. 271-321 in Holomorphic curves in symplectic geometry, edited by M. Audin and J. Lafontaine, Progr. Math. 117, Birkhäuser, Basel, 1994. MR 1274934 Zbl 0802.53001

[Biran 2006] P. Biran, "Lagrangian non-intersections”, Geom. Funct. Anal. 16:2 (2006), 279-326. MR 2007b:53178 Zbl 1099.53054

[Biran and Cieliebak 2002] P. Biran and K. Cieliebak, "Lagrangian embeddings into subcritical Stein manifolds", Israel J. Math. 127 (2002), 221-244. MR 2003g:53151 Zbl 01786380

[Biran et al. 2003] P. Biran, L. Polterovich, and D. Salamon, "Propagation in Hamiltonian dynamics and relative symplectic homology", Duke Math. J. 119:1 (2003), 65-118. MR 2004i:53124 Zbl 1034.53089

[Bourgeois 2003] F. Bourgeois, "A Morse-Bott approach to contact homology", pp. 55-77 in Symplectic and contact topology: interactions and perspectives (Toronto/Montreal, 2001), edited by Y. Eliashberg et al., Fields Inst. Commun. 35, Amer. Math. Soc., Providence, RI, 2003. MR 2004a: 53109 Zbl 1046.57017

[Bourgeois and Oancea 2007] F. Bourgeois and A. Oancea, "An exact sequence for contact and symplectic homology", Preprint, 2007. arXiv 0704.2169

[Cieliebak 2002a] K. Cieliebak, "Handle attaching in symplectic homology and the chord conjecture”, J. Eur. Math. Soc. (JEMS) 4:2 (2002), 115-142. MR 2003d:53153 Zbl 1012.53066

[Cieliebak 2002b] K. Cieliebak, "Subcritical Stein manifolds are split”, Preprint, 2002. arXiv math. DG/0204351

[Cieliebak and Mohnke 2004] K. Cieliebak and K. Mohnke, "Punctured holomorphic curves and Lagrangian embeddings", Preprint, 2004.

[Cieliebak and Oancea 2008] K. Cieliebak and A. Oancea, Work in preparation, 2008.

[Cieliebak et al. 2008] K. Cieliebak, U. Frauenfelder, and A. Oancea, Work in preparation, 2008.

[Duistermaat 1976] J. J. Duistermaat, "On the Morse index in variational calculus", Advances in Math. 21:2 (1976), 173-195. MR 58 \#31190 Zbl 0361.49026

[Ekholm 2001] T. Ekholm, "Differential 3-knots in 5-space with and without self-intersections", Topology 40:1 (2001), 157-196. MR 2001h:57033 Zbl 0964.57029

[Eliashberg 1990] Y. Eliashberg, "Topological characterization of Stein manifolds of dimension > 2”, Internat. J. Math. 1:1 (1990), 29-46. MR 91k:32012 Zbl 0699.58002

[Eliashberg and Gromov 1991] Y. Eliashberg and M. Gromov, "Convex symplectic manifolds", pp. 135-162 in Several complex variables and complex geometry, Part 2 (Santa Cruz, CA, 1989), edited by E. Bedford et al., Proc. Sympos. Pure Math. 52, Amer. Math. Soc., Providence, RI, 1991. MR 93f:58073 Zbl 0742.53010

[Eliashberg et al. 2000] Y. Eliashberg, A. Givental, and H. Hofer, "Introduction to symplectic field theory”, Geom. Funct. Anal. Special Volume, Part II (2000), 560-673. GAFA 2000 (Tel Aviv, 1999). MR 2002e:53136 Zbl 0989.81114

[Floer 1988a] A. Floer, "Morse theory for Lagrangian intersections", J. Differential Geom. 28:3 (1988), 513-547. MR 90f:58058 Zbl 0674.57027

[Floer 1988b] A. Floer, "A relative Morse index for the symplectic action”, Comm. Pure Appl. Math. 41:4 (1988), 393-407. MR 89f:58055 Zbl 0633.58009

[Floer 1988c] A. Floer, "The unregularized gradient flow of the symplectic action", Comm. Pure Appl. Math. 41:6 (1988), 775-813. MR 89g:58065 Zbl 0633.53058 
[Floer 1989a] A. Floer, "Symplectic fixed points and holomorphic spheres", Comm. Math. Phys. 120:4 (1989), 575-611. MR 90e:58047 Zbl 0755.58022

[Floer 1989b] A. Floer, "Witten's complex and infinite-dimensional Morse theory", J. Differential Geom. 30:1 (1989), 207-221. MR 90d:58029 Zbl 0678.58012

[Frauenfelder 2004] U. Frauenfelder, "The Arnold-Givental conjecture and moment Floer homology”, Int. Math. Res. Not. 2004:42 (2004), 2179-2269. MR 2005g:53174 Zbl 1088.53058

[Frauenfelder et al. 2005] U. Frauenfelder, V. Ginzburg, and F. Schlenk, "Energy capacity inequalities via an action selector", pp. 129-152 in Geometry, spectral theory, groups, and dynamics, edited by M. Entov et al., Contemp. Math. 387, Amer. Math. Soc., Providence, RI, 2005. MR 2006h:53091 Zbl 1094.53079

[Geiges 1994] H. Geiges, "Symplectic manifolds with disconnected boundary of contact type", Internat. Math. Res. Notices 1994:1 (1994), 23-30. MR 94m:53042 Zbl 0815.53041

[Geiges 2008] H. Geiges, An introduction to contact topology, Cambridge Studies in Advanced Math. 109, Cambridge University Press, 2008. MR 2008m:57064 Zbl 05265449

[Ginzburg and Gürel 2003] V. L. Ginzburg and B. Z. Gürel, "A $C^{2}$-smooth counterexample to the Hamiltonian Seifert conjecture in $\mathbb{R}^{4}$ ", Ann. of Math. (2) 158:3 (2003), 953-976. MR 2005e:37133 Zbl 1140.37356

[Gompf 2005] R. E. Gompf, "Stein surfaces as open subsets of $\mathbb{C}^{2}$ ”, J. Symplectic Geom. 3:4 (2005), 565-587. MR 2007f:32031 Zbl 1118.32011

[Gromov 1985] M. Gromov, "Pseudoholomorphic curves in symplectic manifolds", Invent. Math. 82:2 (1985), 307-347. MR 87j:53053 Zbl 0592.53025

[Kato 1976] T. Kato, Perturbation theory for linear operators, 2nd ed., Grundlehren der Math. Wissenschaften 132, Springer, Berlin, 1976. MR 53 \#11389 Zbl 0342.47009

[Klingenberg 1995] W. P. A. Klingenberg, Riemannian geometry, 2nd ed., de Gruyter Studies in Math. 1, Walter de Gruyter \& Co., Berlin, 1995. MR 95m:53003 Zbl 0911.53022

[Massey 1969] W. S. Massey, "Proof of a conjecture of Whitney", Pacific J. Math. 31 (1969), 143156. MR 40 \#3570 Zbl 0198.56701

[McDuff 1991] D. McDuff, "Symplectic manifolds with contact type boundaries", Invent. Math. 103:3 (1991), 651-671. MR 92e:53042 Zbl 0719.53015

[McDuff and Salamon 1998] D. McDuff and D. Salamon, Introduction to symplectic topology, 2nd ed., Oxford Math. Monogr., The Clarendon Press, Oxford University Press, New York, 1998. MR 2000g:53098 Zbl 1066.53137

[McDuff and Salamon 2004] D. McDuff and D. Salamon, J-holomorphic curves and symplectic topology, Amer. Math. Soc. Colloq. Publ. 52, Amer. Math. Soc., Providence, RI, 2004. MR 2004m: 53154 Zbl 1064.53051

[McLean 2007] M. McLean, "Lefschetz fibrations and symplectic homology", Preprint, 2007. arXiv 0709.1639

[Morita 1975] S. Morita, "A topological classification of complex structures on $S^{1} \times S^{2 n-1}$ ", Topology 14 (1975), 13-22. MR 53 \#9237 Zbl 0301.57010

[Morse 1996] M. Morse, The calculus of variations in the large, Amer. Math. Soc. Colloq. Publ. 18, Amer. Math. Soc., Providence, RI, 1996. Reprint of the 1932 original. MR 98f:58070 ZBL 0011. 02802

[Poźniak 1999] M. Poźniak, "Floer homology, Novikov rings and clean intersections", pp. 119-181 in Northern California Symplectic Geometry Seminar, edited by Y. Eliashberg et al., Amer. Math. Soc. Transl. Ser. 2 196, Amer. Math. Soc., Providence, RI, 1999. MR 2001a:53124 Zbl 0948.57025 
[Rabinowitz 1978] P. H. Rabinowitz, "Periodic solutions of Hamiltonian systems", Comm. Pure Appl. Math. 31:2 (1978), 157-184. MR 57 \#7674 Zbl 0358.70014

[Robbin and Salamon 1993a] J. Robbin and D. Salamon, “The Maslov index for paths", Topology 32:4 (1993), 827-844. MR 94i:58071 Zbl 0798.58018

[Robbin and Salamon 1993b] J. Robbin and D. Salamon, "Phase functions and path integrals", pp. 203-226 in Symplectic geometry, edited by D. Salamon, London Math. Soc. Lecture Note Ser. 192, Cambridge Univ. Press, 1993. MR 95i:58076 Zbl 0809.58007

[Robbin and Salamon 1995] J. Robbin and D. Salamon, "The spectral flow and the Maslov index", Bull. London Math. Soc. 27:1 (1995), 1-33. MR 96d:58021 Zbl 0859.58025

[Robbin and Salamon 1996] J. Robbin and D. Salamon, "Feynman path integrals on phase space and the metaplectic representation", Math. Z. 221:2 (1996), 307-335. MR 98f:58051 Zbl 0848.58008

[Salamon 1999] D. Salamon, "Lectures on Floer homology", pp. 143-229 in Symplectic geometry and topology (Park City, UT, 1997), edited by Y. Eliashberg and L. Traynor, IAS/Park City Math. Ser. 7, Amer. Math. Soc., Providence, RI, 1999. MR 2000g:53100 Zbl 1031.53118

[Salamon and Zehnder 1992] D. Salamon and E. Zehnder, "Morse theory for periodic solutions of Hamiltonian systems and the Maslov index", Comm. Pure Appl. Math. 45:10 (1992), 1303-1360. MR 93g:58028 Zbl 0766.58023

[Schwarz 1993] M. Schwarz, Morse homology, Progress in Math. 111, Birkhäuser Verlag, Basel, 1993. MR 95a:58022 Zbl 0806.57020

[Viterbo 1999] C. Viterbo, "Functors and computations in Floer homology with applications, I", Geom. Funct. Anal. 9:5 (1999), 985-1033. MR 2000j:53115 Zbl 0954.57015

[Wasserman 1969] A. G. Wasserman, "Equivariant differential topology", Topology 8 (1969), 127150. MR 40 \#3563 Zbl 0215.24702

[Weber 2002] J. Weber, "Perturbed closed geodesics are periodic orbits: index and transversality", Math. Z. 241:1 (2002), 45-82. MR 2003j:53137 Zbl 1037.53060

[Yau 2004] M.-L. Yau, "Cylindrical contact homology of subcritical Stein-fillable contact manifolds”, Geom. Topol. 8 (2004), 1243-1280. MR 2005g:53172 Zbl 1055.57036

[Ziller 1977] W. Ziller, "The free loop space of globally symmetric spaces", Invent. Math. 41:1 (1977), 1-22. MR 58 \#31198 Zbl 0338.58007

Received October 4, 2007. Revised September 24, 2008.

\section{KAI CIELIEBAK}

\section{DEPARTMENT OF MATHEMATICS}

LUDWIG-MAXIMILIAN UNIVERSITY

THERESIENSTRASSE 39

MUNICH, BAVARIA 80333

GERMANY

kai@mathematik.uni-muenchen.de

URS ADRIAN FRAUENFELDER

DEPARTMENT OF MATHEMATICS

LUDWIG-MAXIMILIAN UNIVERSITY

THERESIENSTRASSE 39

MUNICH, BAVARIA 80333

GERMANY

frauenf@mathematik.uni-muenchen.de 